\title{
Zur Überlieferung mittelgriechischer Taktiker.
}

I.

Wer sich mit der hslichen Überlieferung der Taktik des Kaisers Leo eingehender befaBt, wird unschwer die Bemerkung machen können, daB das Werk einer wiederholten Überarbeitung unterzogen worden ist. Allerdings nicht einer Überarbeitung, die an den wesentlichen Inhalt angesetzt hat: die taktischen Regeln und strategischen Anordnungen haben schon seit dem sogen. Maurikios keine erhebliche Wandlung erlitten, ein Umstand also, der dem kaiserlichen Kompilator seine Aufgabe wesentlich erleichtert hat. Aber in sprachlicher und stilistischer Hinsicht war umsomehr zu tun. Wohl rühṃt sich unser Leo „der Weise"

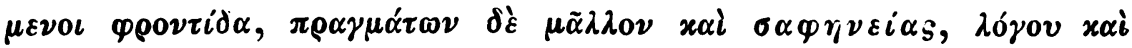

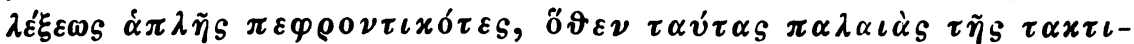

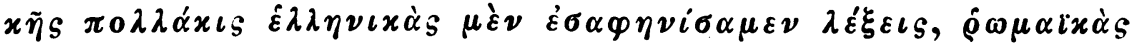

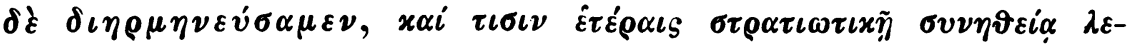

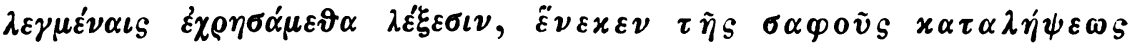
$\tau \tilde{\omega} \nu \varepsilon^{\varepsilon} \nu \tau v \gamma \chi \alpha \nu o ́ v \tau \omega \nu$ (ed. Meurs-Lami VI. p. 537), aber diese teilweise vom sogen. Maurikios eingegebene Willensäußerung (ed. Scheffer [Upsaliae 1664] p. 3) muB, wie die Folge lehrt, denn doch nicht gehörig in die Tat umgesetzt worden sein. Das läBt sich auch leicht beweisen.

Die Hss der Taktik des Kaisers Leo zerfallen nämlich in drei Klassen, ebenso, wie die Sammlung der griechischen Kriegsschriftsteller in drei Familien. ${ }^{1}$ ) Von diesen drei Klassen hat die erste ihren be.

1) Vgl. dazu K. K. Müller, Eine griech. Schrift über Seekrieg (Würzburg 1882) S. 30 u. f., und M. Jähns, Gesch. der Kriegswissenschaften I (MünchenLeipzig 1889) S. 5-8. Danach wird die erste Familie durch Paris. gr. suppl. 607 rertreten. Sie enthält Poliorketiker, wie Athenaios, Bito, Hero und Exzerpte aus Historikern. Die zweite Familie, reprüsentiert durch den Laur. LV 4, enthält mit Ausnahme von Aeneas, allgemein taktische oder Schriften über Feldkrieg (im Ambros. B 119 sup. treten hinzu noch Schriften über den Seekrieg, im Ambros. C. 265 inf. noch die Konstantinische Taktik). Die dritte Familie repräsentieren Vatic. gr. 1164 und der mit dem Paris.' gr. 2442 zusammengehörige Barberino- 
deutendsten Vertreter in dem berühmten Mediceo-Laurentianus gr. LV 4, den bekanntlich K. K. Müller in der Festschrift für L. Urlichs (Würzburg 1880) auf S. 106-108 ziemlich genau beschrieben hat. Diese Hs [F], die Lami seiner Ausgabe zugrunde gelegt hat, gibt den Text in unstreitig sorgfältiger Gestalt wieder, und können wir, abgesehen von den unvermeidlichen Abschreibefehlern des Librarius im ganzen und großen mit der Überlieferung, wie sie in $F$ vorliegt, ganz gut zufrieden sein. Diesem Umstande ist es zu verdanken, wenn die Ausgabe Lamis, nach der gewöhnlich zitiert wird, die meisten Forscher hinlänglich befriedigt hat, und den Wunsch nach einer neuen Ausgabe nicht besonders hat rege werden lassen. Ob mit Recht, wird sich aus dem Folgenden ergeben.

In $F$ fehlen folgende Partien: XIII $11 \mu i \alpha \nu$ i] [p. 696 Z. 34] -

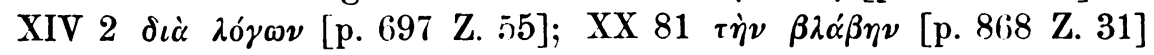

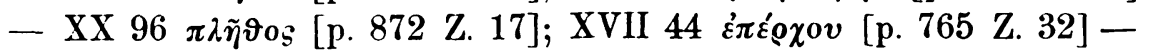

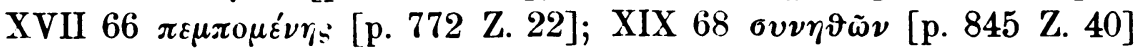

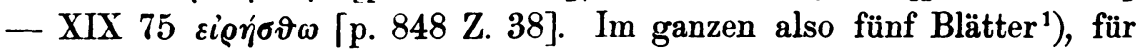
deren Ausfall ein anderer Ersatz gesucht werden muB. Zum Glück besitzt die Wiener Hofbibliothek im Codex Philol. gr. 275 saec. X exeuntis $[V]$ eine Leo-Hs, die hier teilweisen Ersatz schafft. Diese kaum weniger ausgezeichnete $\mathrm{Hs}$ hat von besagten Partien alle, außer p. 868, 31-872, 17, für die wieder eine auf die Rezension ron $F$ oder $V$ zurückgehende jüngere Abschrift herangezogen werden muB. Diese beiden Hss $F$ und $V$ gehen jedenfalls auf ein und denselben Archetypus zurück, aus $\operatorname{dem} F$ früher abgeschrieben ist, $V$ etwas später, als im Archetypus schon mannigfache Randkorrekturen gemacht worden waren. Um diese meine Behauptung zu erhärten, weise ich auf den kritischen Apparat meiner Ausgabe des XVIII. Kapitels hin, deren Varianten wie

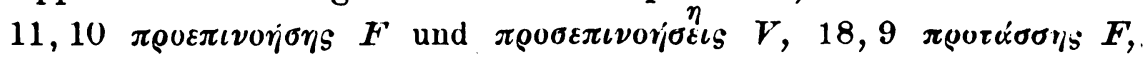

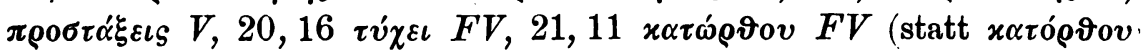

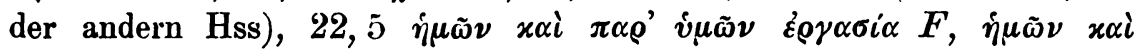
$\pi \alpha \varrho \dot{v} \mu \tilde{\omega} \nu$ ह่ $\varrho \gamma \alpha \sigma i \alpha V$ (während $x \alpha i$ in den andern Hss fehlt), 23, 7

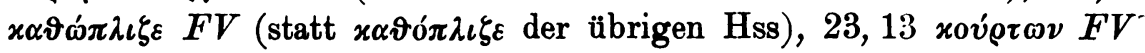

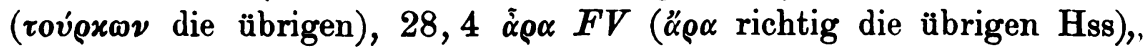

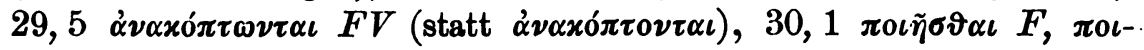

Vaticanus II 97. Hier sind nicht nur taktische Schriften mit poliorketıscienı vereinigt, sondern es treten noch hinzu der von mir herausgegebene Anonymus; saec. $X$ und die unter dem Namen des Kaisers Nikephoros Phokas gehende: Schrift De velitatione bellicu.

1) Die rote Randnote Lamis im Codex, sowie seine FuBnote in der Ausgabe, gibt (zu XVII 44) unrichtig den Ausfall nur eines Blattes an. 


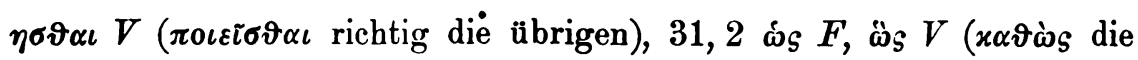

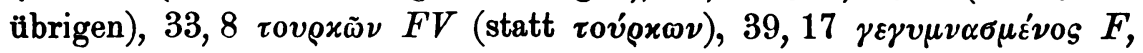

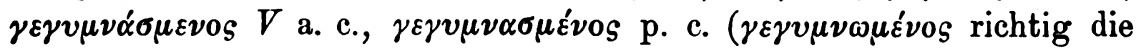
übrigen Hss) usw. den Beweis erbringen, daB $F$ und $V$ auf das innigste zusammengehören, zugleich aber auch, daB sie gegenüber den Repräsentanten der zwei anderen Klassen einen ungleich reineren und ursprünglicheren Text bewahrt haben.

Dessen ungeachtet müssen auch die Repräsentanten der zwei anderen Klassen in einem kritischen Apparat untergebracht werden. Aus dreierlei Gründen. Erstens, weil mit ihrer Hilfe doch mitunter die Fehler der Vorlage von $F V$ aufgedeckt und verbessert werden können, zweitens, weil sie dort, wo sie den Text paraphrasieren, zum Verständnisse des Originales erheblich beitragen (- manchmal auch leicht irreführen -), drittens, weil das konsequente und planmäBige Vorgehen der Überarbeiter in unendlich lehrreicher Weise klar macht, wie die Byzantiner im 9. und 10. Jahrhundert obsolete Wörter und Wendungen in ihren Schriften mit moderneren vertauscht haben. Es wird wohl in der byzantinischen Literatur nicht sobald einen zweiten Text geben, dessen Geschichte dies in so lehrreicher Art zu veranschaulichen imstande wäre, als der von Kaiser Leo ausgeschriebene, und weiterhin noch umgestaltete sogenannte Maurikios.

Von der zweiten Klasse der Hss haben wir in dem vorzüglichen Ambrosianus B 119 sup. (A) saec. XII (über den ebenfalls K. K. Müller uns Aufschluß gibt in der Abhandl.: Eine griech. Schrift über Seekrieg, Würzburg 1882 , S. 18-39) den besten Vertreter. Dieser Codex, der näher zu $V$ steht als zu $F$, enthält nicht nur Leos Taktik in zusammengedrängterer Fassung, sondern in geänderter Fassung auch die des sogenannteu Maurikios. Zur Bekräftigung der Richtigkeit dieser Behauptung sei mir gestattet einige Parallelstellon nach don Hss $F$ (bezieh. $V$ ) und $A$ unten wiederzugeben. Doch, was Leos Taktik anbetrifft, soll dies nicht ohne Heranziehung der Vertreter der dritten Klasse geschehen, die im Vaticanus 1164 gr. (saec. XI) $[K]$ und dem Barberinianus II 97 (saec. XI) $\left[P_{1}\right]$ ihre vollwertigsten Repräsentanten gefunden hat. Diese setzen die Überarbeitung, die in $A$ vorliegt, schon als eine gegebene Tatsache voraus, gehen aber in der Behandlung des Textes um ein klein weniges weiter, was wohl in der hier unten folgenden Partie nicht so sehr in die Augen sticht, aus dem Apparate des von wir herausgegebenen XVIII. Kapitels aber ganz unzweifelhaft hervorgeht. Wesentlichere Zusütze sind im ambrosianischen Maurikios, sowie in der lconiniscber Taktik nit gesperrten Lettern gedruckt. 
Sogen. Maurikios Sogen. Maurikios (florentinische Fassung) (ambrosian. Fassung)

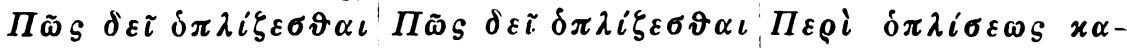

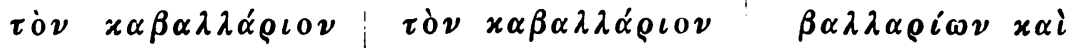

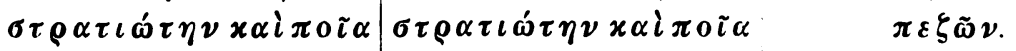

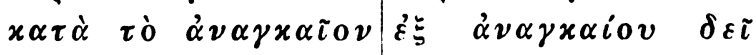
$5 \quad \varepsilon i \delta \eta x \tau \tilde{\alpha} \sigma \vartheta \alpha \iota ;$ $x \tau \tilde{\alpha} \sigma \vartheta \alpha \iota ;$

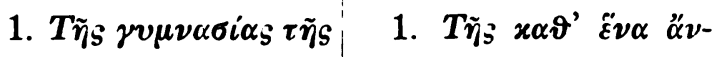

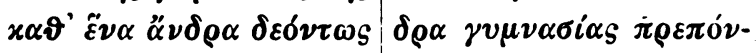

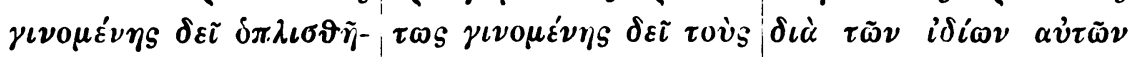

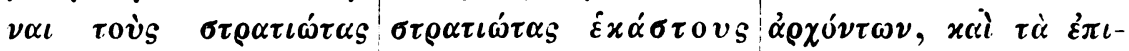

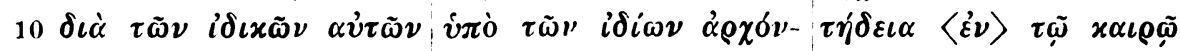

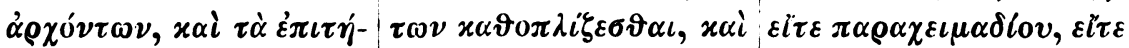

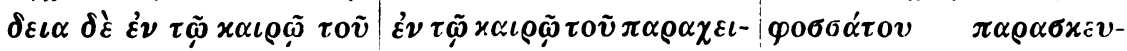

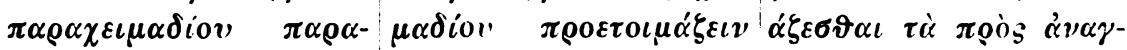

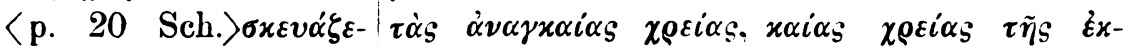

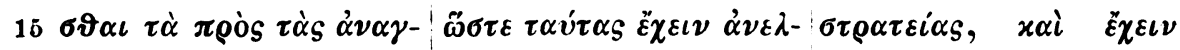

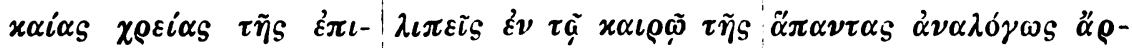

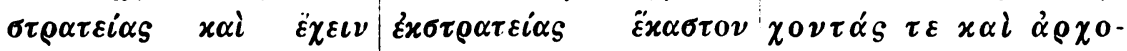

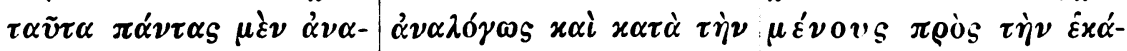

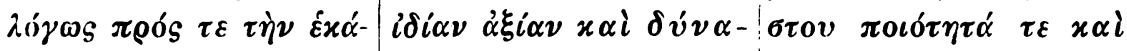

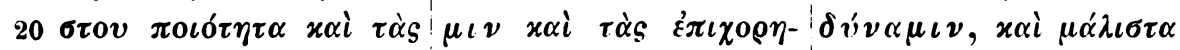

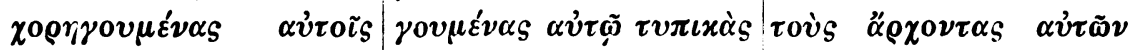

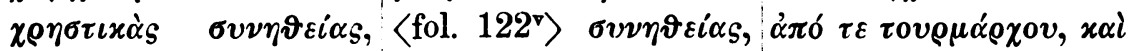

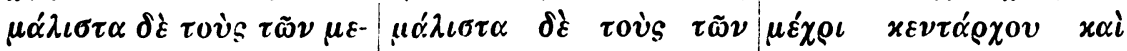

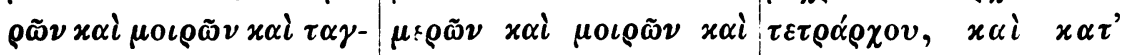

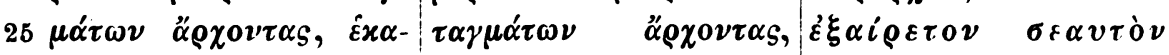

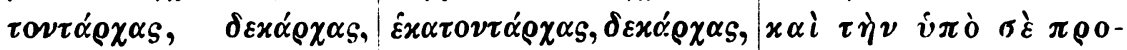

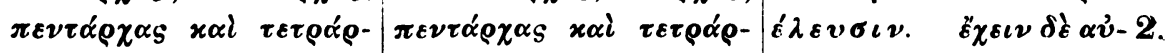

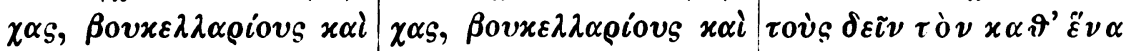

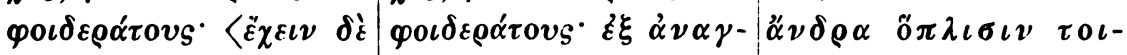

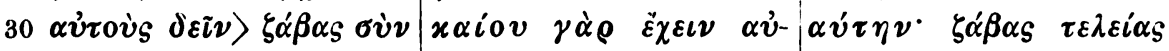

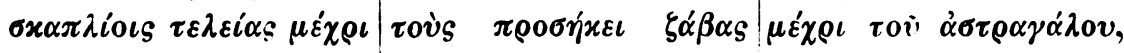

$7 x \alpha \vartheta \varepsilon \nu \alpha F \| 22 x \rho v-$ $\sigma \iota x \dot{\alpha} s F$; emendavit Sch ef-

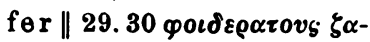
$\beta \alpha_{s} F \mid \varepsilon_{z} x . \delta . \alpha \dot{v} \tau . \delta$. inseruit

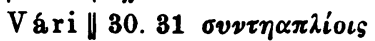
videtur exstare in $\boldsymbol{F}$
$4 \dot{\xi} \xi \alpha \nu \alpha \gamma x \alpha i o v \quad A \| 15$. $16 \dot{\alpha} \nu \varepsilon \lambda \lambda_{\imath} \pi \tilde{\omega} s \quad A \| 29.30 \dot{\xi} \xi$ $\alpha \nu \alpha \gamma x \alpha i v v A$
$8 \delta \iota \varkappa \tau \tilde{\omega} \nu|\alpha| \alpha \dot{v} \tau \tilde{\nu}$ om. $A \| 10$ Év Vári inseruit \| $11 \pi \alpha \rho \dot{\alpha} x \varepsilon \varphi \mu \alpha \delta i o v$ $A\|13 \pi \rho \circ V\| 13.14 \dot{\alpha} \nu \alpha \gamma-$ $x \alpha i \alpha_{S} R \| 14.15 \xi \gamma x \rho \alpha \tau \varepsilon i \alpha_{S}$ $P_{1} R\|18 \pi \rho 0 \sigma \tau \dot{\nu} \nu\| 24.25$

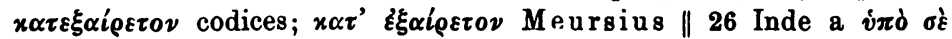
usque ad $30 \tau \varepsilon \lambda \varepsilon i \alpha s$ litterae parum lucidae sunt in $V \mid i \pi v \sigma \varepsilon R$, i $\pi \dot{a}$

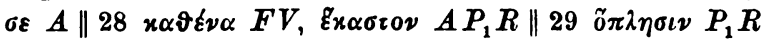




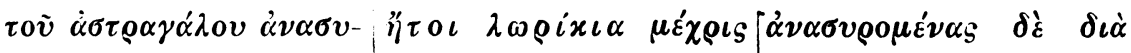

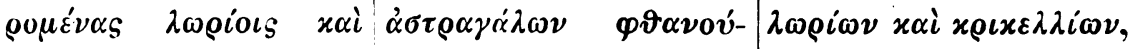

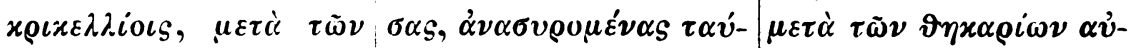

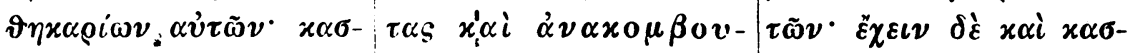

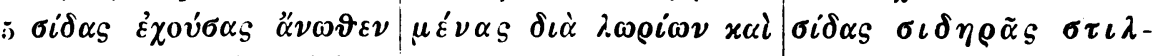

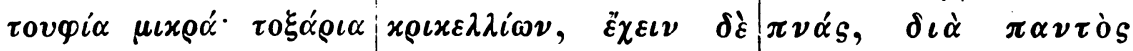

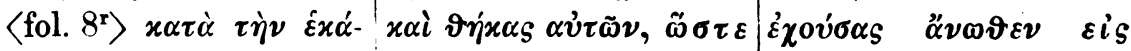

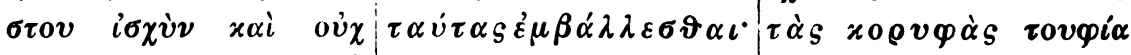

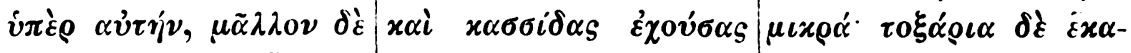

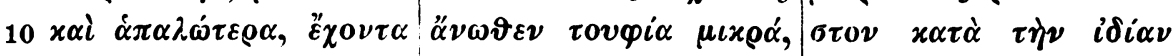

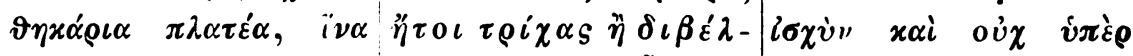

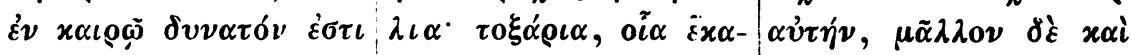

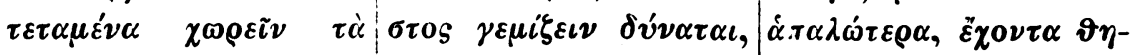

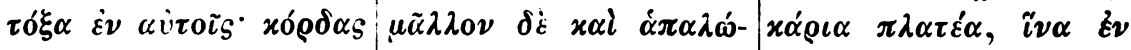

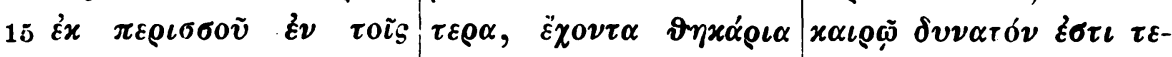

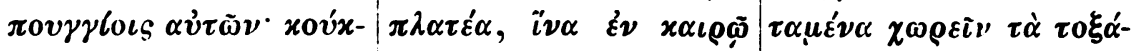

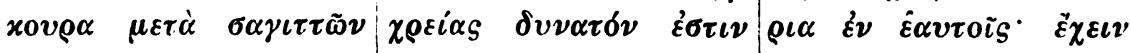

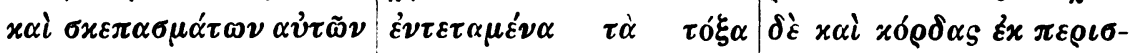

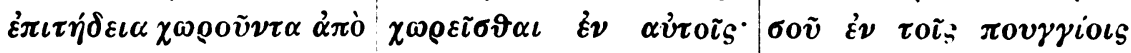

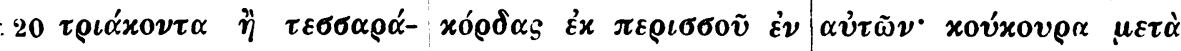

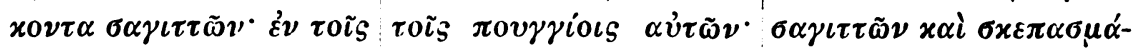

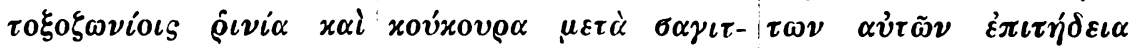

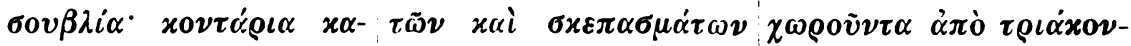

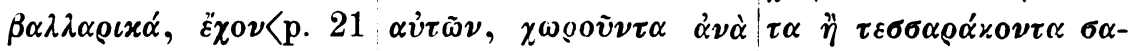

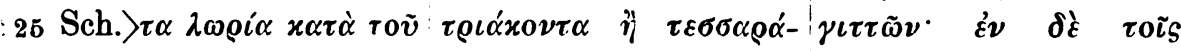

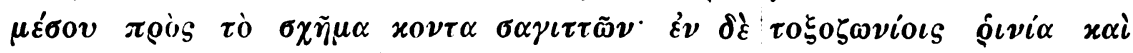

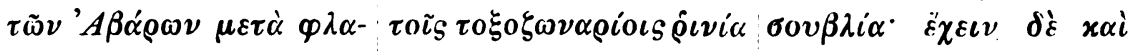

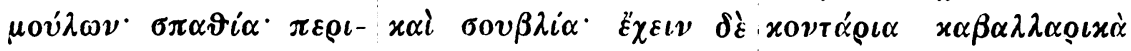

8 ov่x' F $\| 10 \dot{\alpha} \pi \alpha \lambda \dot{\omega}-$

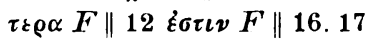

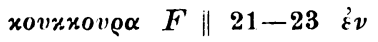

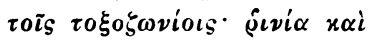

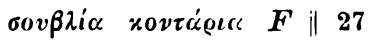

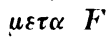

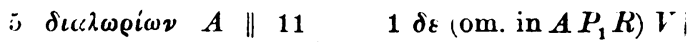
$\delta_{1} \beta * \dot{\varepsilon} \lambda_{1} \alpha \quad\left(\beta\right.$ quoque in $\delta i \dot{\alpha} \boldsymbol{F} V\left\|2 x \rho \iota x \varepsilon \lambda_{i} \omega \nu A\right\|$

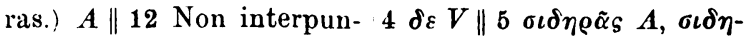
gitur ante $\tau 0 \xi \dot{\alpha} \rho \iota \alpha$ in $\left.A\left\|\rho \dot{\alpha} s F V P_{1} R\right\| 5.6 \sigma \tau \iota \lambda \pi v \dot{\alpha} s\right]$

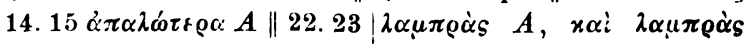

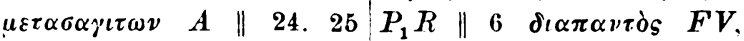

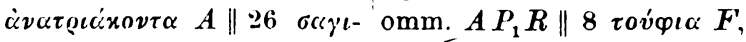
$\tau \omega v A$

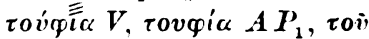

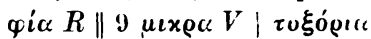

$P_{1}\|\delta \varepsilon V\| 11.12 \dot{v} \pi \varepsilon \rho \alpha v \tau i \eta v\|12 \delta \varepsilon V\| 13 \dot{\alpha} \pi \alpha \lambda \dot{\omega} \tau \varepsilon \rho \alpha P_{1} \| 13.14$

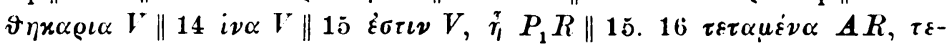

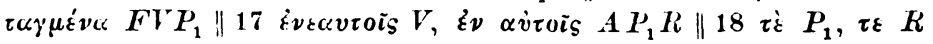
$21 \sigma \alpha \gamma \iota \tau \tau \omega \nu$ F, б« $\bar{\lambda} F P_{1} R\left\|24 \bar{\mu} F, \mu^{\prime} V\right\| 24.25 \sigma \alpha \gamma \iota \tau \tau \omega \nu F, \sigma \alpha_{j}^{\prime} \iota \tau \dot{\omega} \nu A P_{1} R \| 25 \delta \varepsilon V$

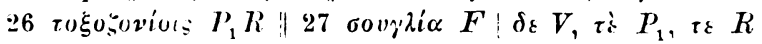




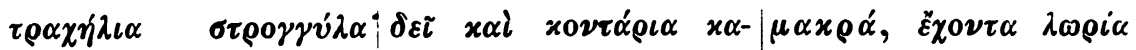

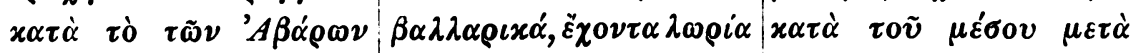

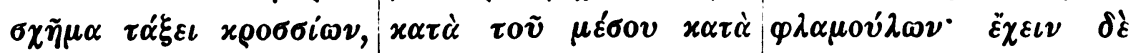

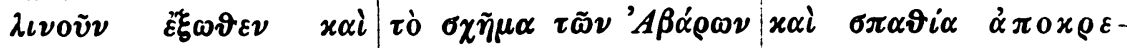

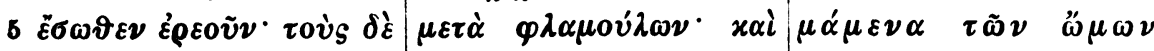

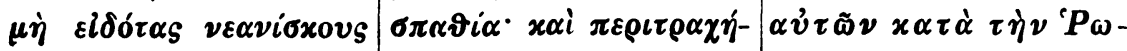

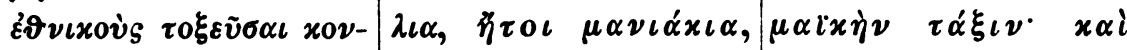

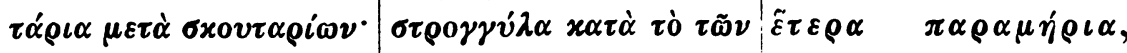

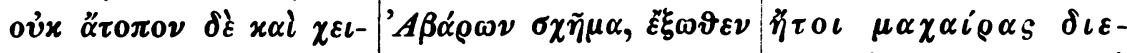

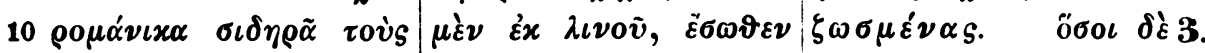

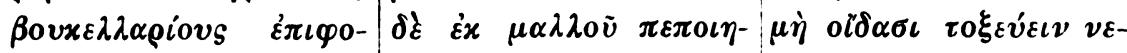

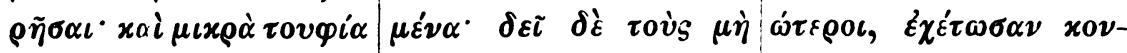

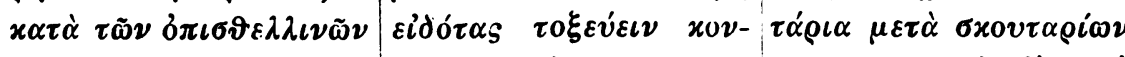

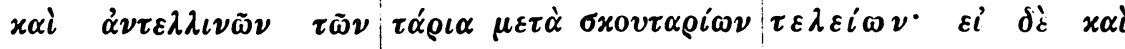

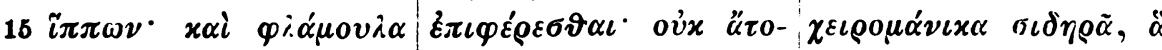

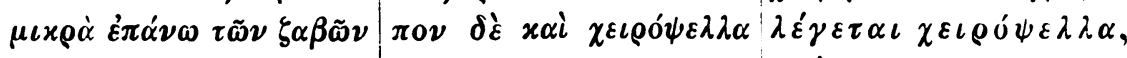

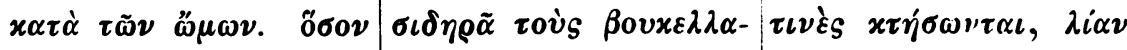

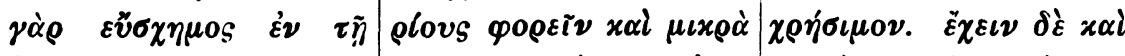

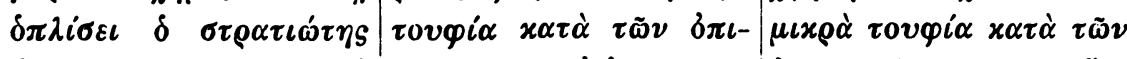

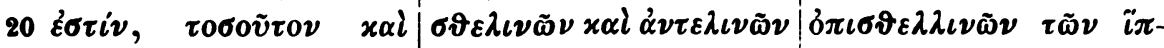

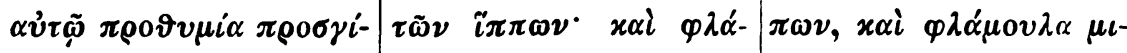

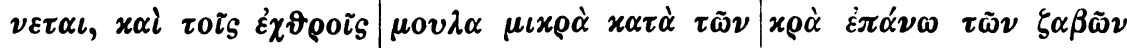
$\delta \varepsilon\llcorner\lambda i \alpha$.

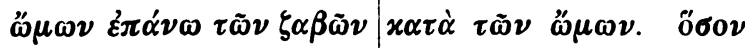

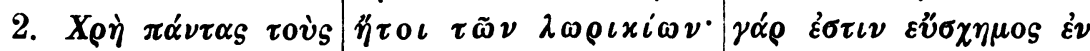

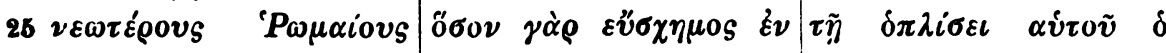

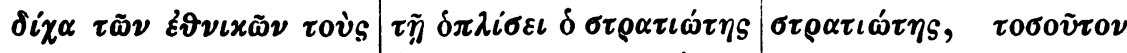

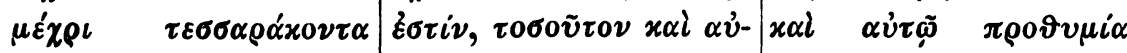

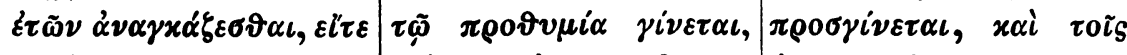

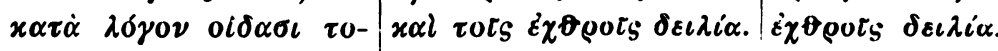

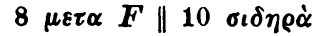
$F\|11.12 \xi \pi \iota v \circ \tilde{\eta} \sigma \alpha \iota \quad F, 17 \sigma \iota \delta \eta \rho \dot{\alpha} A\| 19.20 \delta \pi \iota \sigma \vartheta \varepsilon-$

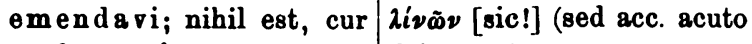
conferam $\varepsilon \pi \iota \nu 0 \eta \tilde{\sigma} \alpha \iota$ infra deleto) $\boldsymbol{A}$

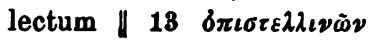

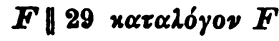

$1 \mu \alpha x \rho \alpha \mid \mu \iota x \rho \dot{\alpha} x \alpha \beta$.

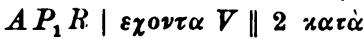

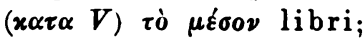
emendavi | $\mu \varepsilon \tau \alpha V \| 3 \delta \varepsilon$ $V \|$ 4. $5 \alpha \pi 0 x \rho \varepsilon \mu \alpha \mu \varepsilon v \alpha V$

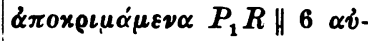
$|\tau \operatorname{cov} V| x \alpha \tau \alpha \tau \eta \nu V|| 7 \tau \alpha \xi\llcorner\nu$

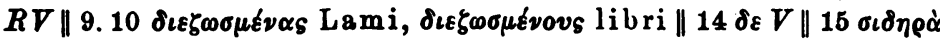

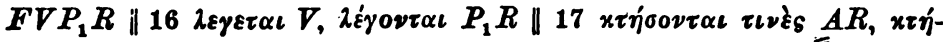

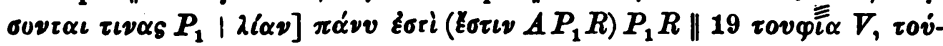

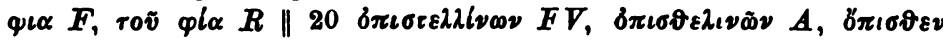

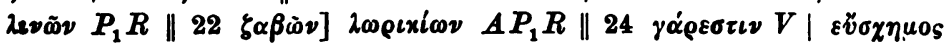

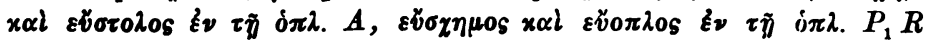
$25 \alpha \dot{v} \tau o \tilde{v}$ Vári, $\alpha \dot{v} \tau o \tilde{v}$ codd. 
$\xi \varepsilon \tilde{v} \sigma \alpha \iota, \varepsilon \ddot{i} \varepsilon \varepsilon \varepsilon \varepsilon \tau \rho i \omega s, \tau 0 \tilde{v}$

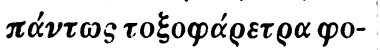

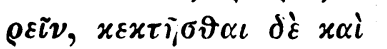

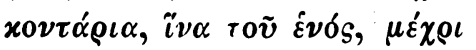

2. $\Delta \varepsilon \tilde{\imath} \delta \dot{\varepsilon} \pi \alpha \dot{\nu} \tau \alpha_{S} \tau o \dot{v} s$

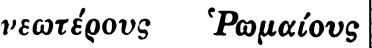

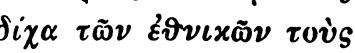
$\tau \varepsilon \sigma \sigma \alpha \rho \alpha ́ x 0 \nu \tau \alpha$

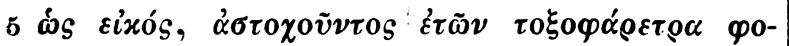

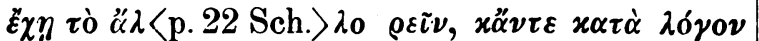

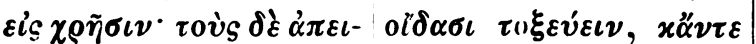

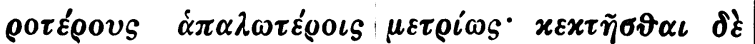

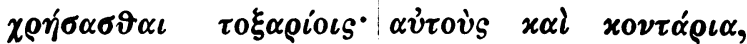

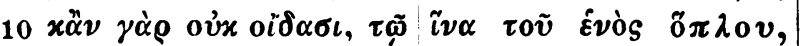

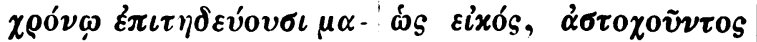

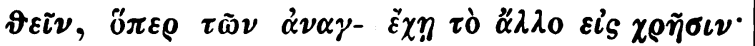

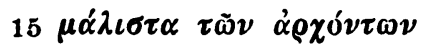

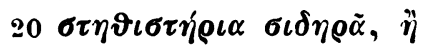
$25 \tau \tilde{\omega} \nu, \mu \alpha^{\prime} \lambda \iota \sigma \tau \alpha\left\langle\delta^{\prime} \dot{\xi}\right\rangle \tau \tilde{\omega} \nu$

:30

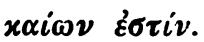

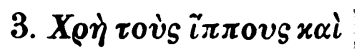

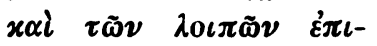
$\lambda \varepsilon \dot{x} \tau \omega \nu \quad \pi \rho 0 \mu \varepsilon \tau \omega \pi i \delta\llcorner\alpha$

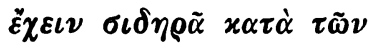

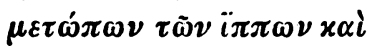

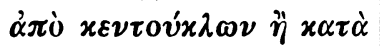

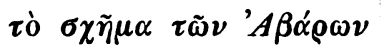

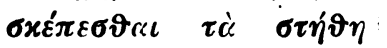

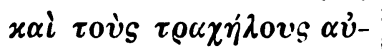

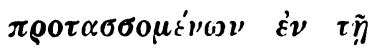
$\mu \alpha \dot{\chi} \chi \cdot$. oi $\delta \dot{\alpha} \alpha \dot{\alpha} \pi \varepsilon \iota \cos _{\varepsilon \rho \rho \iota} \dot{\alpha} \pi \alpha-$

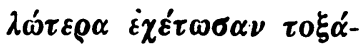

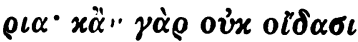
〈fol. 123 $\left.{ }^{\mathrm{r}}\right\rangle \tau 0 \xi \varepsilon v \varepsilon \varepsilon \nu$,

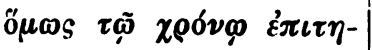

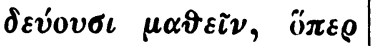

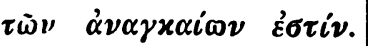

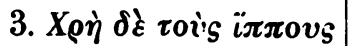

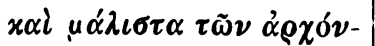
$\tau \omega \nu \quad x \alpha i \quad \tau \tilde{\omega} \nu \quad \lambda 0 \iota \pi \tilde{\omega} \nu$

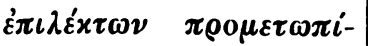

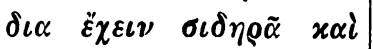

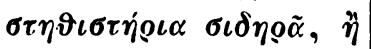

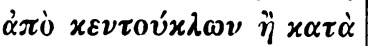

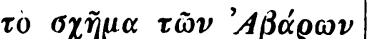
$\sigma x \varepsilon \dot{\pi \varepsilon \sigma \vartheta \alpha \iota} \tau \dot{\alpha} \sigma \tau \dot{\eta} \vartheta \eta$ $\tau o \grave{s} s \rho \alpha \chi \dot{\eta} \lambda o v s \alpha \dot{v} \tau \grave{\omega} \nu$,

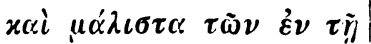
$\mu \alpha \dot{\chi !) ~ \pi \varrho о \tau \alpha \sigma \sigma о \mu \varepsilon ́ v \omega \nu . ~}$

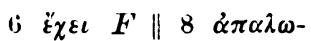

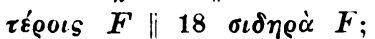
item infra $\| \mathbf{2 5} \delta$ : Vári inseruit

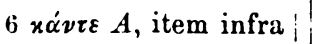

$\varepsilon l \delta v \nu \alpha \tau \dot{o} \nu \delta \dot{\varepsilon} x \alpha i 4$.

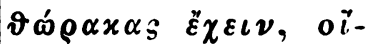
$\tau \iota \nu \varepsilon \subseteq x \alpha \lambda \circ \tilde{v} \nu \tau \alpha \iota \nu \tilde{v} \nu$ $x \lambda \iota \beta \alpha^{\prime} \nu \iota \alpha, x \alpha i \quad \alpha \dot{v} \tau \dot{\alpha}$ $\sigma \tau \iota \lambda \pi \nu \dot{\alpha} x \alpha i \lambda \alpha \mu \pi \rho \alpha \dot{~}$ $x \alpha i \pi \varepsilon \rho \iota x \nu \eta \mu i \delta \alpha s$, $\tilde{\alpha} \tau \iota \nu \alpha \quad \lambda \varepsilon \dot{\varepsilon} \gamma \tau \alpha \iota \quad \nu \tilde{v} \nu$ $\pi \circ \delta o ́ \psi \varepsilon \lambda \lambda \alpha \cdot x \alpha i \pi \tau \varepsilon \rho-$

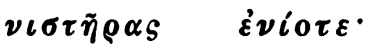

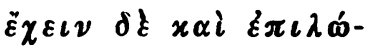
$\rho \iota x \alpha, o ̋ \tau \varepsilon \chi \rho \varepsilon i \alpha, \varepsilon \pi \varepsilon v-$ $\delta v \delta \mu \varepsilon \nu \alpha . \quad \pi \alpha \dot{\nu} \alpha_{S} 5$.

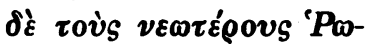

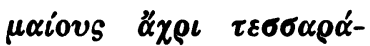

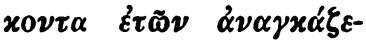

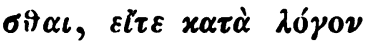

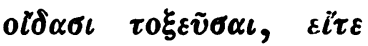

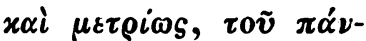

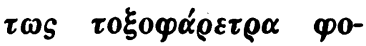

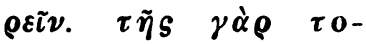
$\xi \varepsilon i \alpha s \pi \alpha \nu \tau \varepsilon \lambda \tilde{\sigma} s \dot{\alpha} \mu \varepsilon-$ $\lambda \eta \vartheta \varepsilon i \sigma \eta s \quad x \alpha i \delta \iota \alpha \pi \varepsilon-$

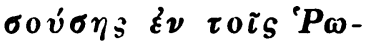
$\mu \alpha i o \iota s \tau \dot{\alpha} \pi 0 \lambda \lambda \dot{\alpha} \nu \tilde{v} \nu$ $\varepsilon i \omega \vartheta \varepsilon \sigma \varphi \alpha^{\prime} \lambda \mu \alpha \tau \alpha \gamma i-$

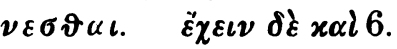

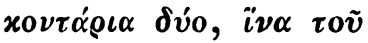

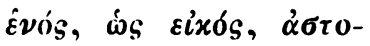

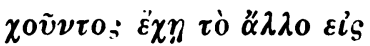
$\chi \varrho \tilde{\eta} \sigma \iota \nu . \quad \tau o \dot{v} s \delta \dot{\varepsilon} \alpha \dot{\alpha} \varepsilon$ -

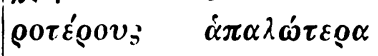

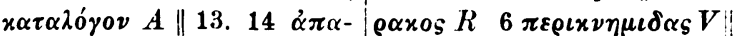

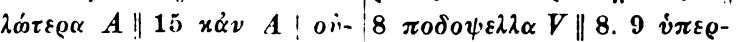

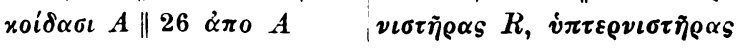
$P_{1} ;$ postea interpungitur in

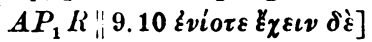

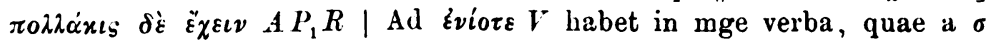
vel $\varepsilon$ incipiunt $\tau \varepsilon$-que finiuntur $\|10 \delta \varepsilon x \alpha i V\| 11$ ô $\tau \varepsilon \chi \rho . ~ \varepsilon \pi$.] $\varepsilon \pi \varepsilon v \delta v o ́ \mu \varepsilon v \alpha$

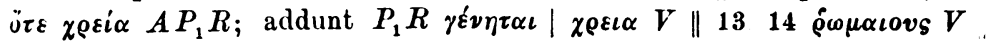

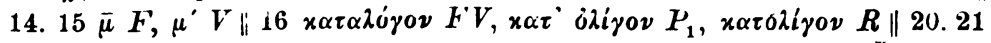

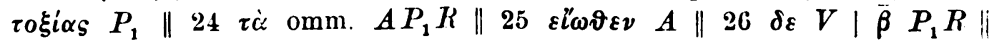

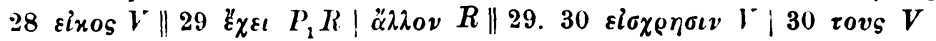




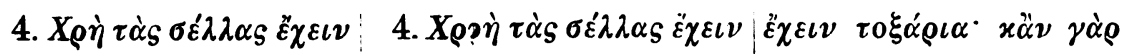

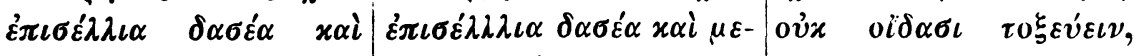

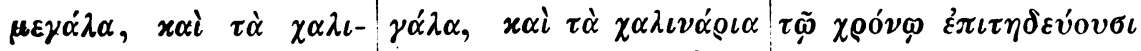

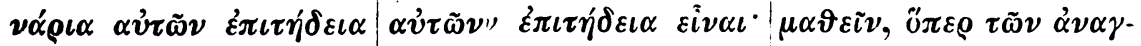

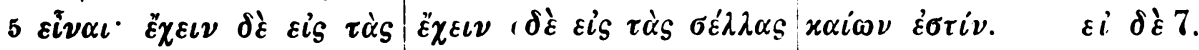

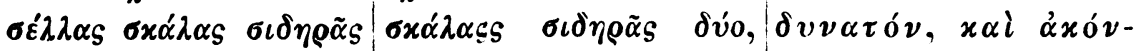

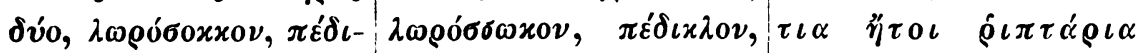

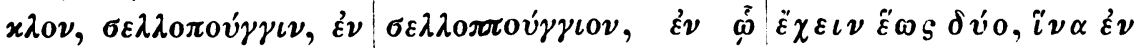

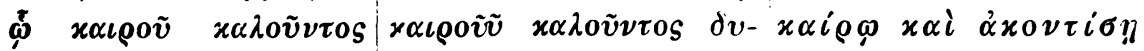

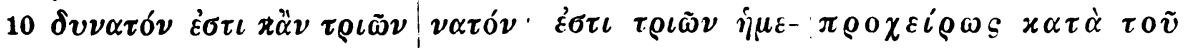

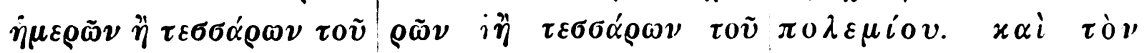

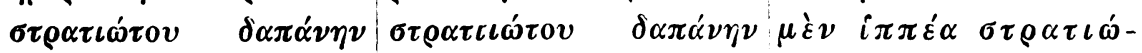

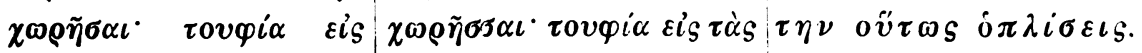

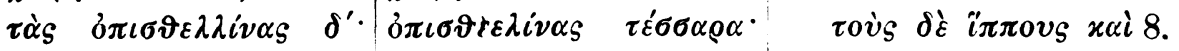

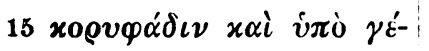

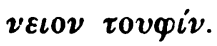

5. X९่̀ $\tau \dot{\alpha} i \mu \alpha \dot{\tau} \tau \iota \alpha \alpha \dot{v} \tau \tilde{\omega} \nu$

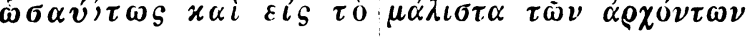

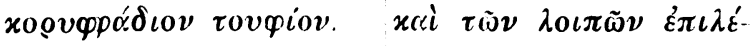

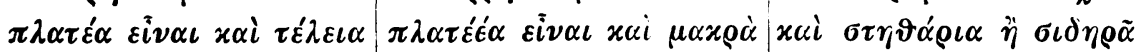

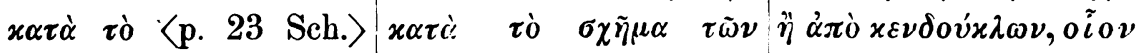

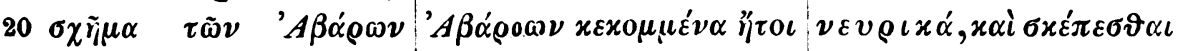

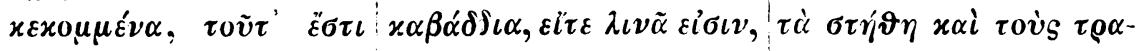

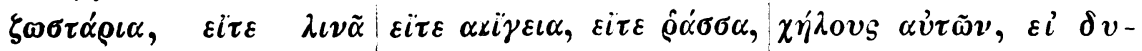

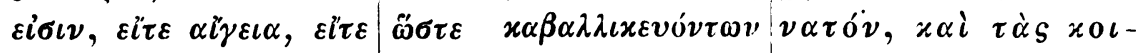

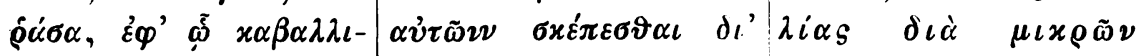

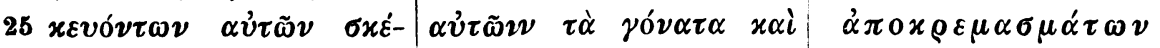

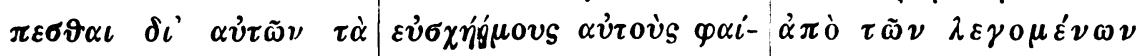

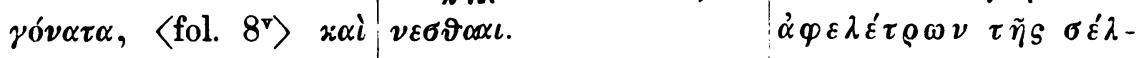

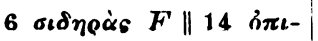

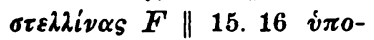

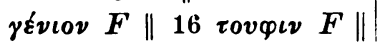

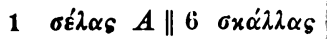

$1 x \dot{\alpha} \nu F A P_{1} R, x \dot{\alpha} \nu V_{1}$ $A \| 21 \iota \lambda \iota v \dot{\alpha} A$

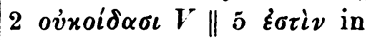
$F$ in fine versus non

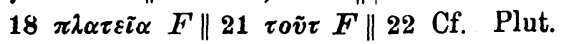

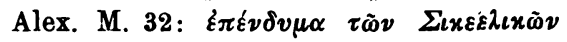

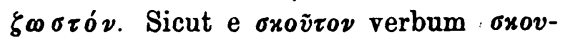
$\tau \alpha \rho \iota \nu \nu$, ita exortum esse puto e verbo $\zeta \omega \sigma \tau o ́ v$ pro substantivo usitato veerbum $\zeta \omega \sigma \tau \alpha \rho \iota 0 \nu$. Noli itaque vocabulum $\zeta \zeta \check{\sigma} \sigma \rho \alpha$

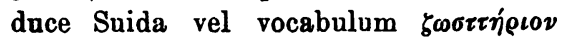
ducibus Ps.-Philoxeno et gr. papyrris in M. Brit. a Kenyon propositis in teextum

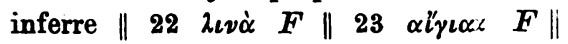
$26 \alpha v \tau \tilde{\omega} \boldsymbol{v} F$

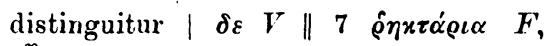

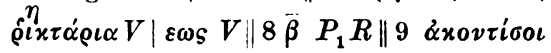

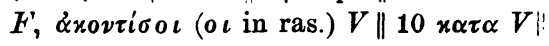
$10.11 \tau \tilde{\omega} \nu \pi 0 \lambda \varepsilon \mu i \omega \nu A P_{1} R, 12 \mu \varepsilon \nu V \mid \sigma \tau \rho \alpha-$ $\tau \iota \omega \tau \eta \nu V, \sigma \tau \rho \alpha \tau \iota \dot{\nu} \nu R$, quod et $P_{1}$ habet (sed ante correcturam) $\| 14$ rovs $\delta \varepsilon V \|$

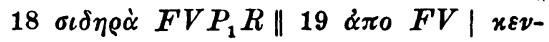

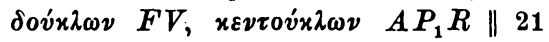
Verba $\tau \dot{\alpha} \sigma \tau \eta^{\prime} \vartheta \eta \ldots \tau \rho \alpha \chi \eta^{\prime} \lambda o v s$ evanuerunt in $V \| 22$ Verba $\alpha \dot{v} \tau \tilde{\omega} \nu$. . . xocגias etiam in mge a $m^{2}$ in $V \| 24 \delta \iota \alpha A V \mid$ $\mu \alpha x \rho \tilde{\omega} \nu P_{1} R\|26 \dot{\alpha} \pi 0 V \mid \lambda \varepsilon \gamma \sigma \mu \varepsilon \nu \omega \nu V\|$

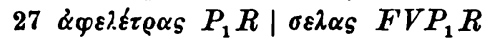


$\varepsilon \dot{v} \sigma \chi \dot{r}_{\mu} \mu v_{S} \alpha \dot{v} \tau o \grave{v} s$ $\varphi \alpha i-$ $\nu \varepsilon \sigma \vartheta \alpha \iota$.

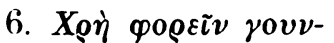

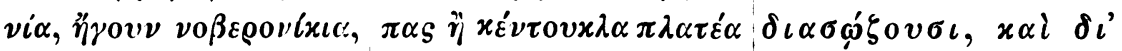

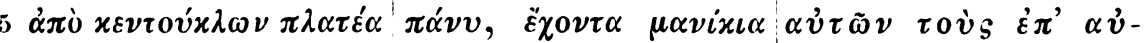

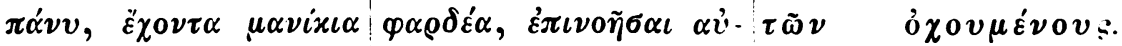

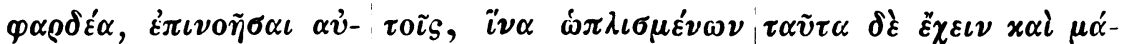

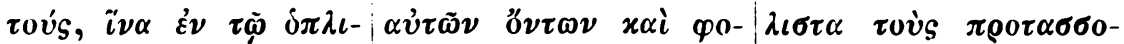

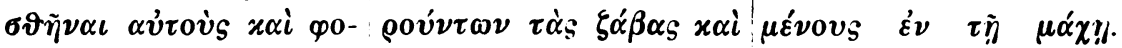

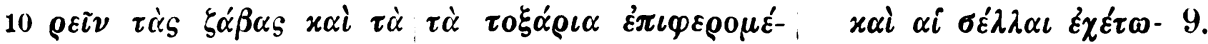

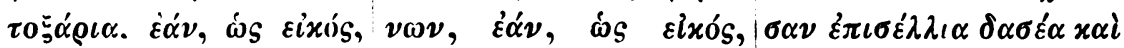

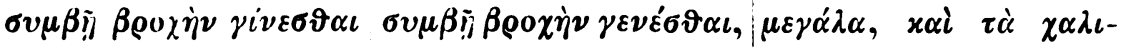

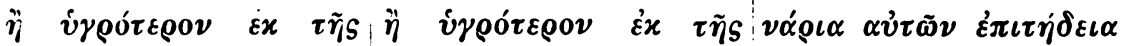

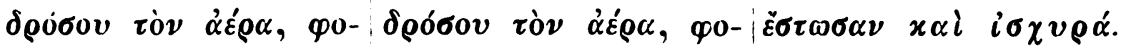

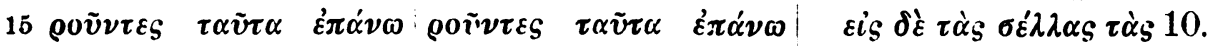

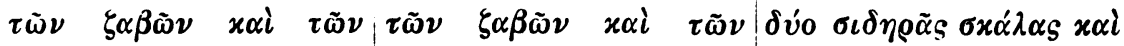

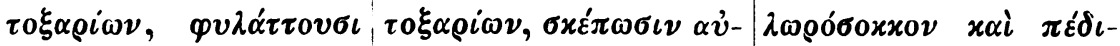

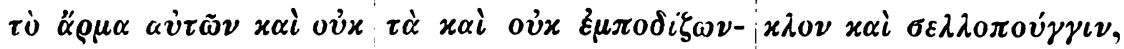

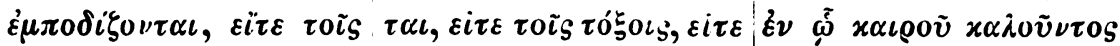

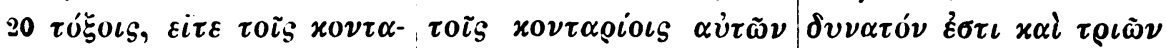

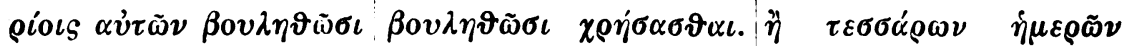

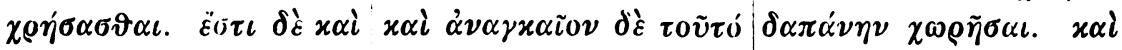

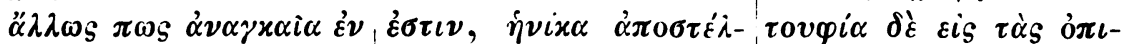

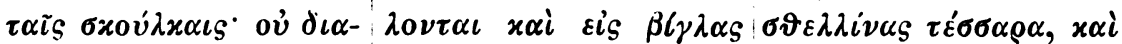

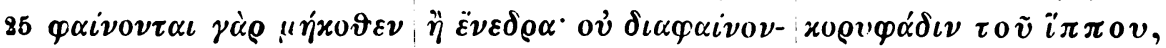

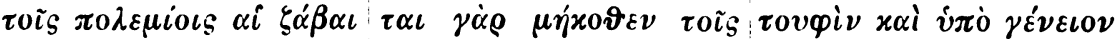

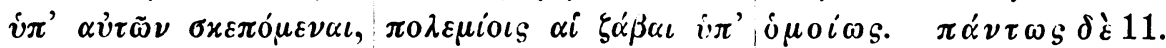

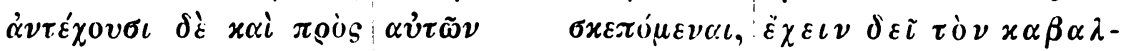

4 , itut gunoberonicia" reddit Sch.; fort. legendum

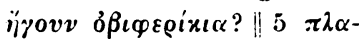
$\tau \varepsilon i \alpha F \| 6$ Post $\mu \alpha \nu$. interpunctione caret $F \quad 7 \varphi \alpha \rho \delta \tilde{\alpha}$ $F: 10 \xi \alpha \beta \alpha_{S} F$ 18 $\tilde{\alpha}^{\prime} \underline{\mu} \alpha F$

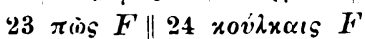

3 Interpunctione omnino caret $A \| 18$ oi $\times \varepsilon u \pi(t-$ si

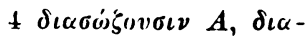

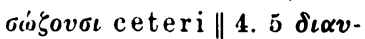

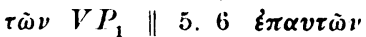
$V P_{1} R\|; \delta \varepsilon V\| 8.9 \pi \rho 0-$

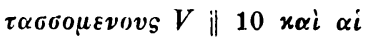

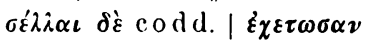
$V \| 12.13 \tau \alpha \chi \alpha \lambda \iota \nu \alpha \dot{\alpha} \rho \alpha(\iota v$ in ras.) $V \| 15 \delta \varepsilon V \mid \sigma \dot{z} \lambda \alpha s$

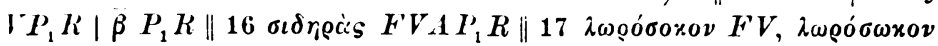

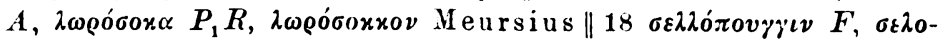

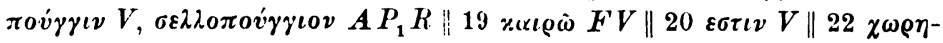

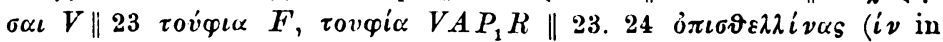

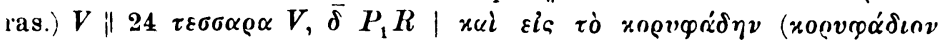

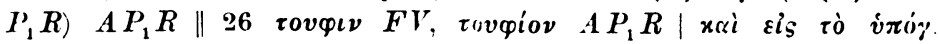
$A P_{1} R \mid$ i $\pi$ ogย́vยเov $F V$ 


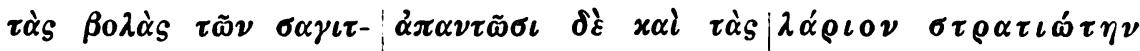
$\tau \tilde{\omega} \nu$.

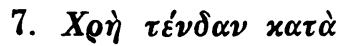

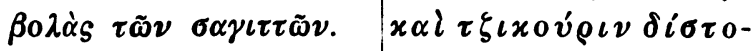

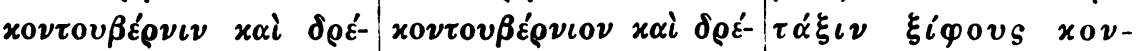

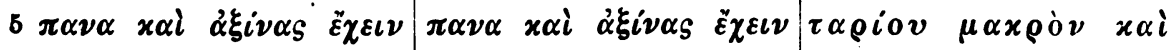

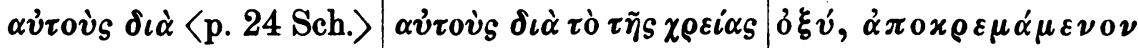

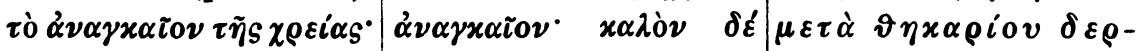

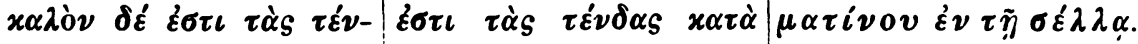

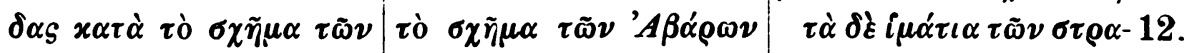

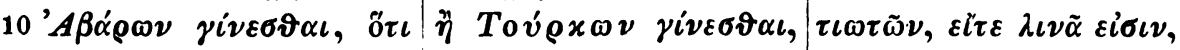

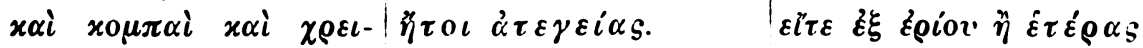

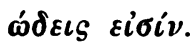

8. Xọ̀ $\alpha \nu \alpha \gamma x \alpha \dot{\xi} \varepsilon \sigma \vartheta \alpha \iota$

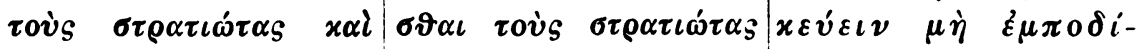

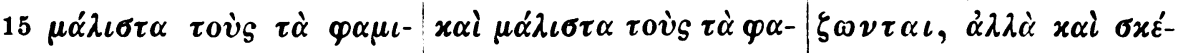

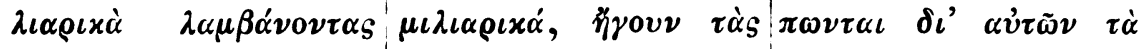

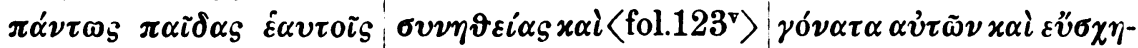

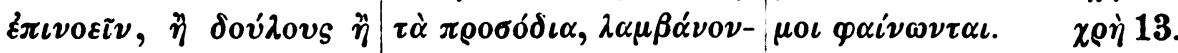

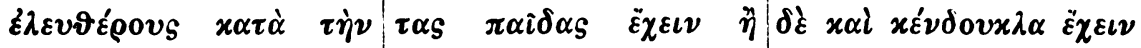

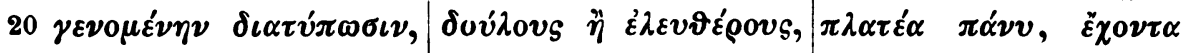

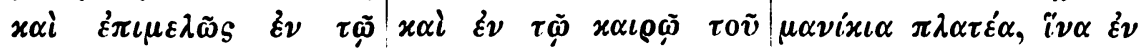

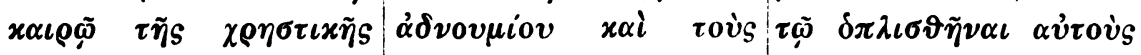

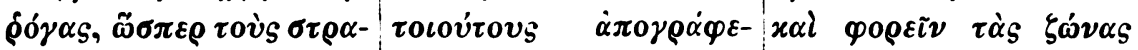

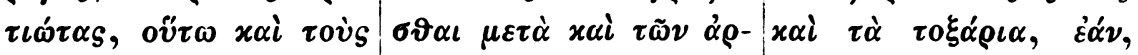

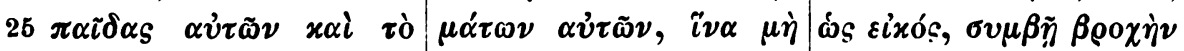

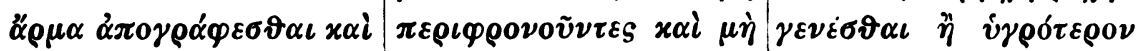

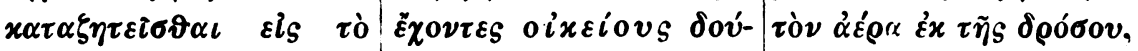

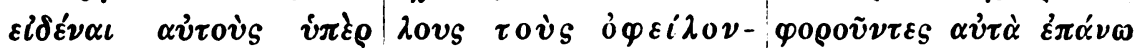

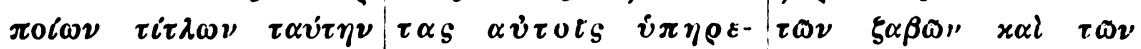

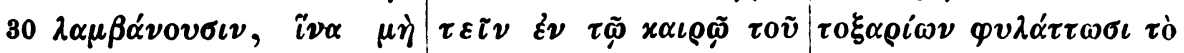

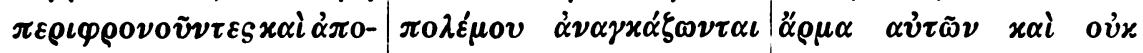

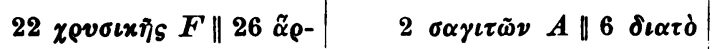
$\mu \alpha F$, et verbum ex verbo $A \mid \chi \rho \varepsilon \tilde{\alpha} \varepsilon A$ Sch. „currum".

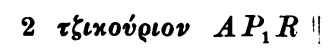

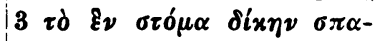

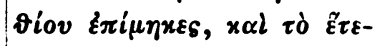

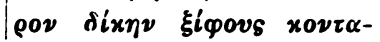

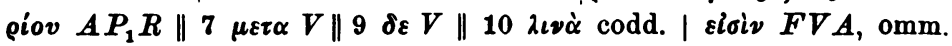

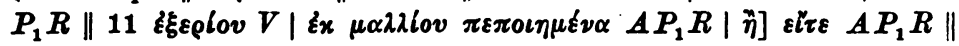

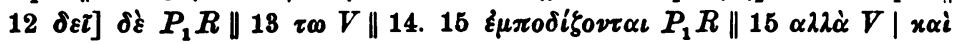

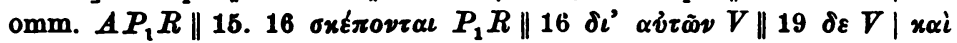

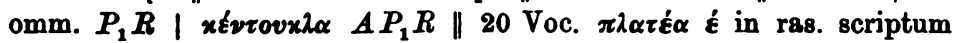
in $V$ uti supra v8. 12, et infra vs. $21 \mid$ Exov $_{1} R\left\|21 \mu \alpha \nu i \alpha ́ x<\alpha A P_{1} R\right\|$

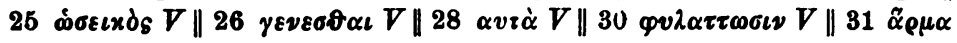
codd. $|\alpha \dot{b \tau \tilde{\omega} \nu} \nabla|$ xal omm. $P_{1} R$ 


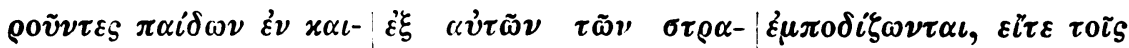

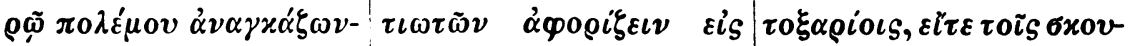

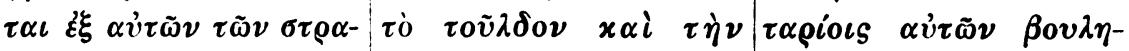

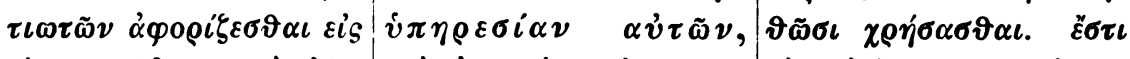

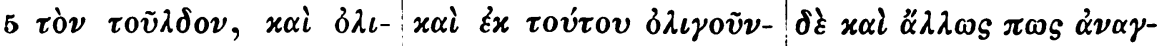

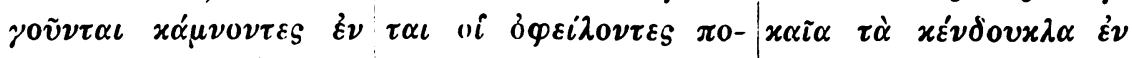
$\tau \tilde{\eta} \mu \alpha^{\prime} \chi \eta^{\circ} \varepsilon i \quad \delta \varepsilon^{\prime} \tau \iota \nu \varepsilon \mathcal{G}, \lambda \varepsilon \mu \varepsilon \tilde{\nu}$.

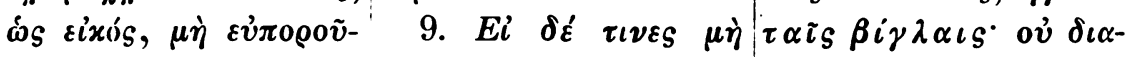

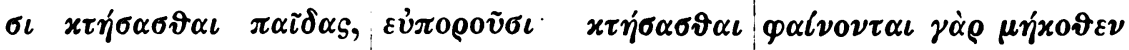

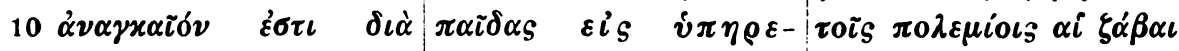

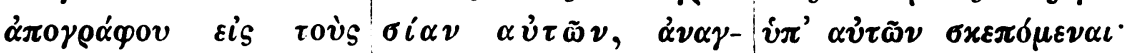

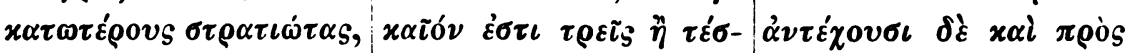

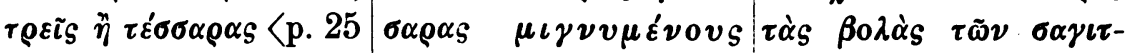

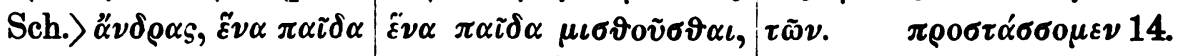

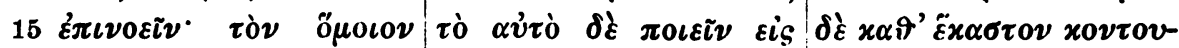

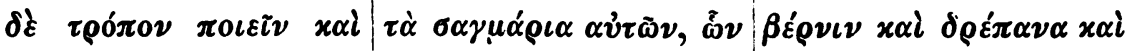

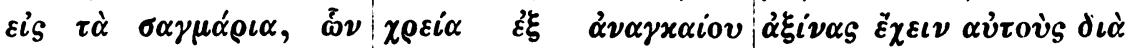

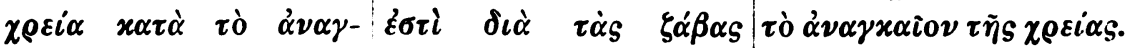

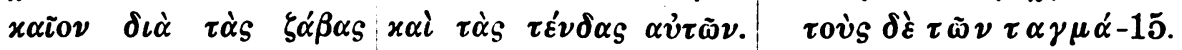

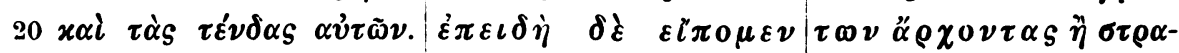

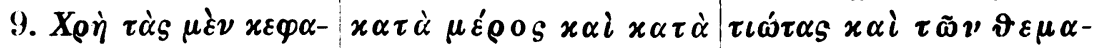

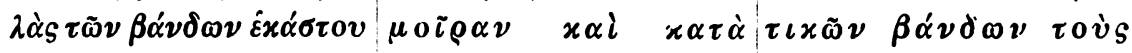

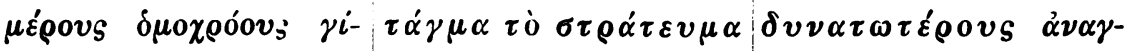

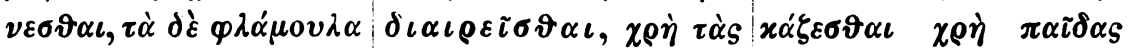

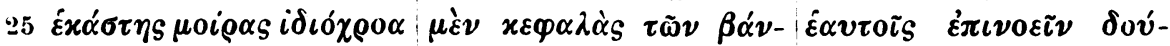

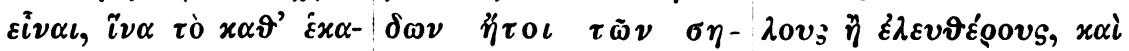

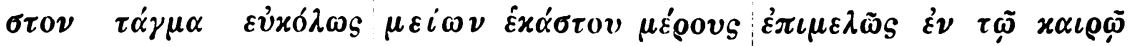

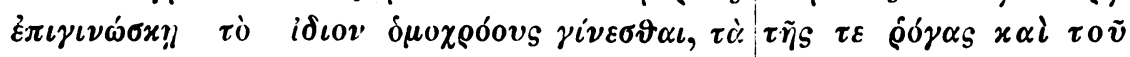
$\beta \dot{\alpha}^{\prime} \nu \delta 0 \nu$.

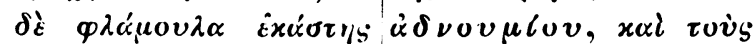

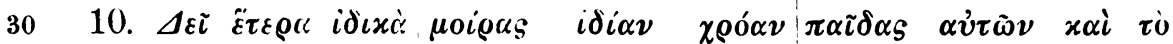

$3 \dot{\xi} \xi \alpha v \tau \dot{\omega} \nu F \| 8 \dot{\omega} \sigma \varepsilon t-$

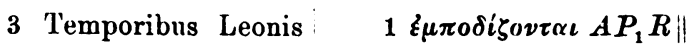

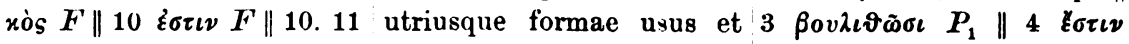

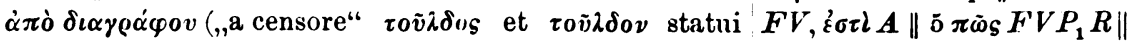

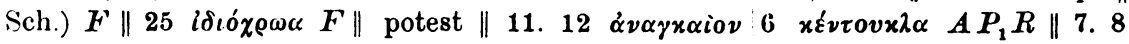
30 i̊ $\iota x \dot{c} F$

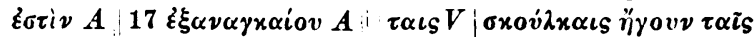

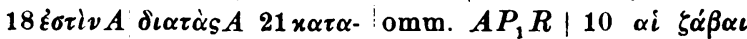

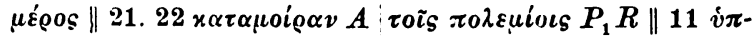
$|\alpha v \tau \tilde{\omega} \nu V| \dot{\alpha} \pi^{\prime} P_{1}|| 12 \dot{\alpha} \pi \alpha \nu-$ $\tau \tilde{\omega} \sigma \iota A P_{1} R \mid \pi \rho \circ V\left\|13.14 \sigma \alpha \gamma \iota \tau \tau \omega \nu F, \sigma \alpha \gamma i \tau \tau \omega \nu V, \sigma \alpha \gamma \iota \tau \dot{\omega} \nu A P_{1} R\right\|$

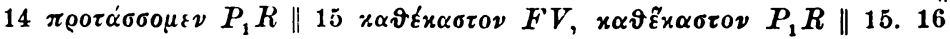

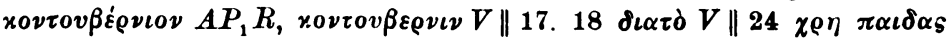

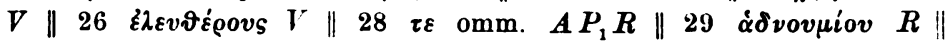
$30 \pi \alpha \iota \delta \alpha_{S} V$ 


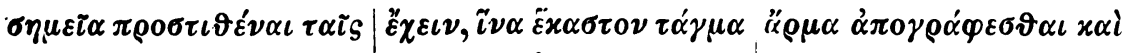

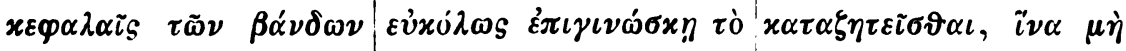

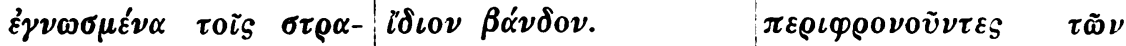

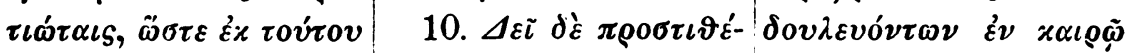

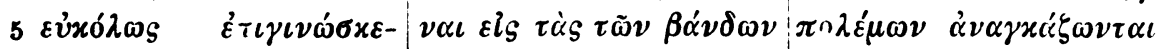

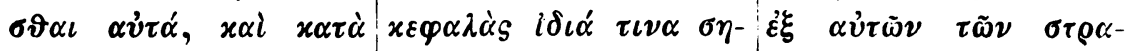

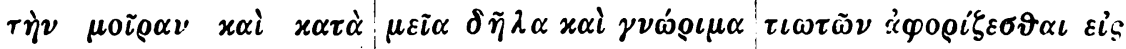
$\tau \grave{o} \tau \dot{\alpha} \gamma \mu \alpha$.

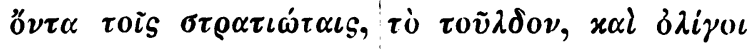

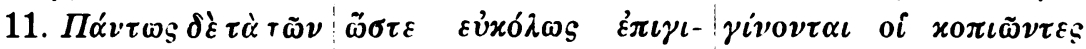

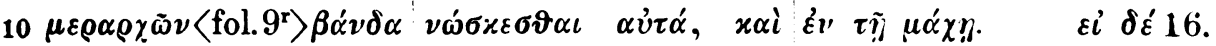

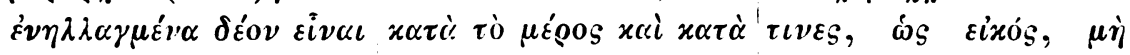

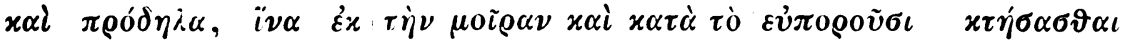

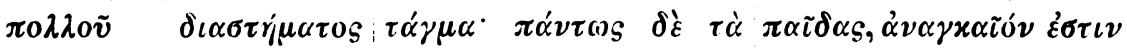

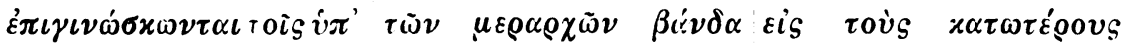

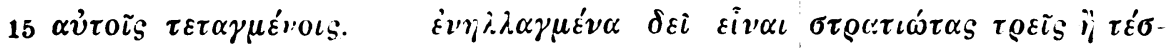

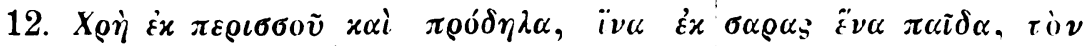

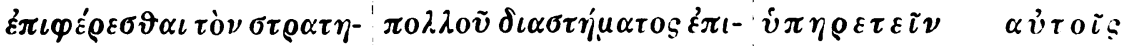

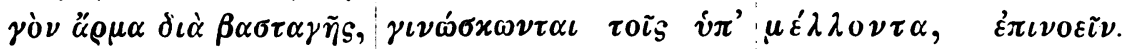

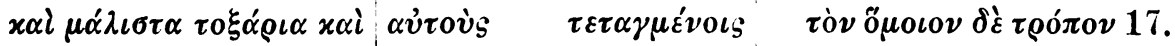
$20 \sigma \alpha \gamma i \tau \tau \alpha$, $i \nu \alpha \tau \tau \tilde{\tau}_{S}, \dot{\omega}_{S} \sigma \tau \rho \alpha \tau \iota \dot{\tau} \tau \iota \iota$ S.

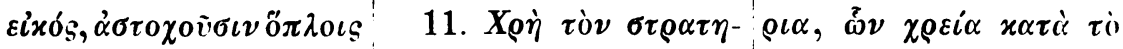

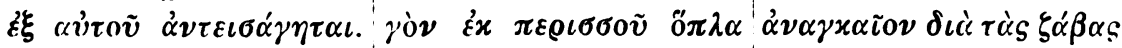

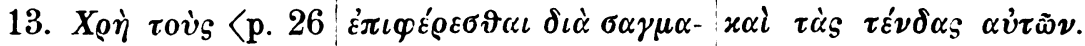

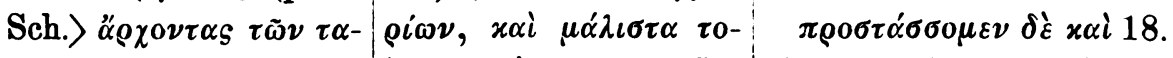

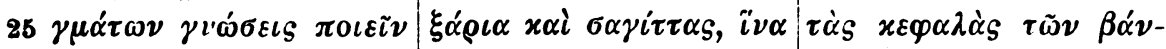

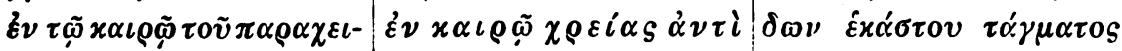

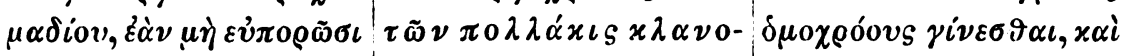

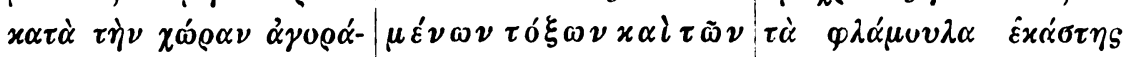

14. $15 \dot{v} \pi^{\prime} \alpha \dot{v} \tau o i s$ non est repudiandum, cf. p. 31 Sch.: $\tau \dot{\eta} \nu \boldsymbol{\nu} \pi \alpha \rho \dot{\alpha} \tau \xi \iota \nu, \tau \dot{\eta} \nu$

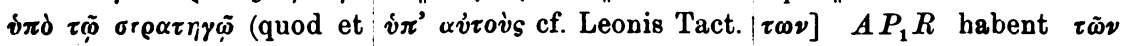

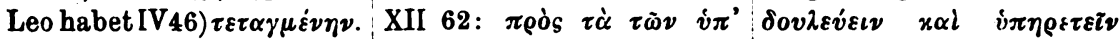

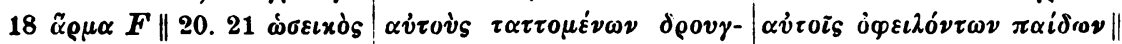
$F \| 22 \dot{\xi} \xi \alpha v \tau o \tilde{v} F|\dot{\alpha} v \tau \varepsilon \iota \sigma-| \gamma \alpha \rho i \omega v$, et Inc. script. saec. X $5 \pi 0 \lambda \dot{\varepsilon} \mu 0 v A P_{1} R \mid \dot{\alpha} v \alpha \gamma x \alpha^{\prime}-$

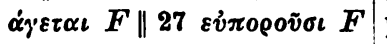

$6 \imath^{\prime \prime} \delta \iota \alpha \tau \iota \nu \grave{\iota} A \| 10$ Post $1 \ddot{\alpha} \rho \mu \alpha V, \ddot{\alpha} \rho \mu \alpha$ c ete ri

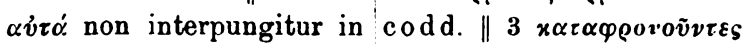

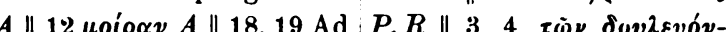

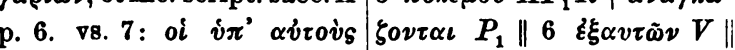

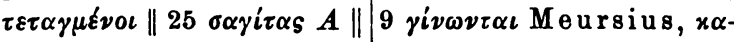
26. $27 \dot{\alpha} \nu \tau \iota \tau \tilde{\omega} \nu A$ $\tau \alpha \lambda_{\iota} \mu \pi \alpha \dot{v} \nu \omega \nu \tau \alpha \iota \quad A, \quad x \alpha \tau \alpha-$ $\left|\lambda \iota \mu \pi \alpha \dot{v} \nu 0 \nu \tau \alpha \iota P_{1} R\right|$ oi $\pi 0 \lambda \varepsilon-$

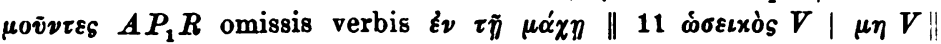

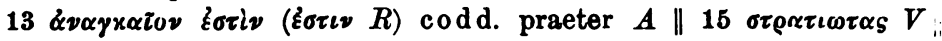
$21 \chi \rho \varepsilon \iota \alpha \nabla\|22 \delta \iota \alpha \tau \dot{\alpha} s \mid\| 23 \tau \varepsilon \nu \tau \alpha_{S} F V\|24 \delta \varepsilon V\| 25.26 \beta \alpha \nu \delta \omega \nu R$ 


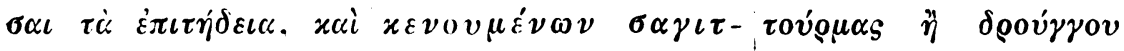

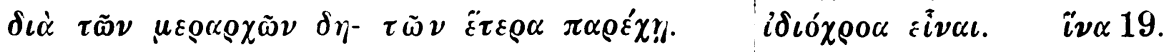

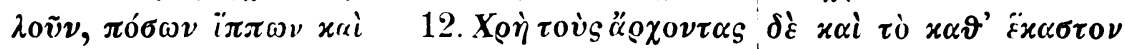

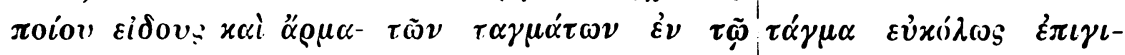

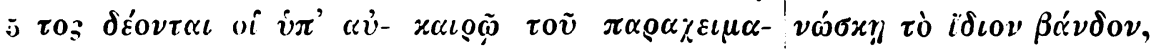

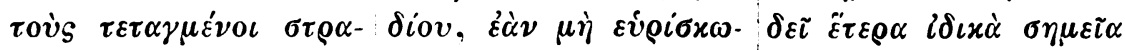

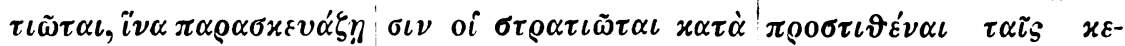

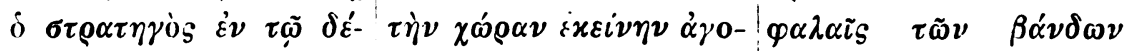

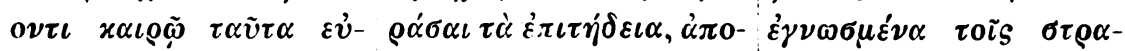

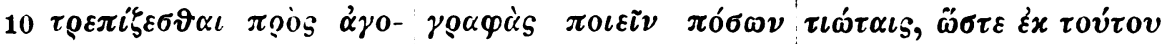

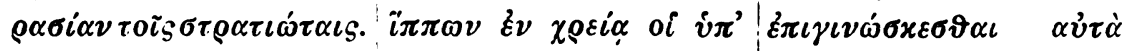

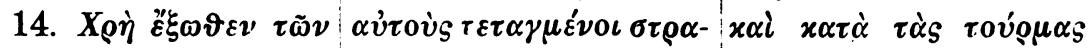

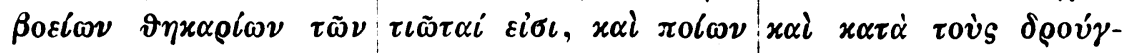

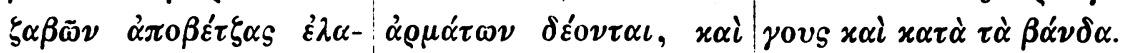

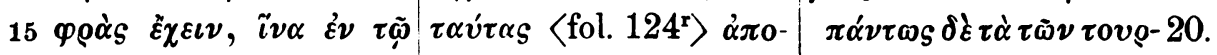

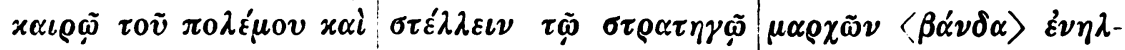

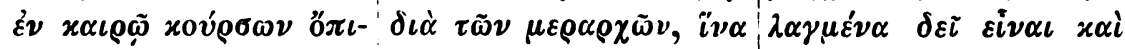

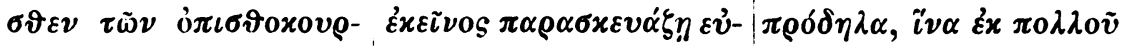

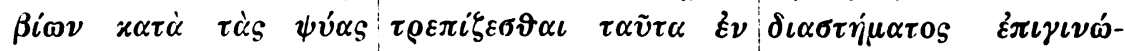

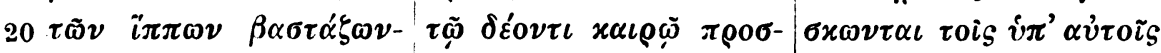

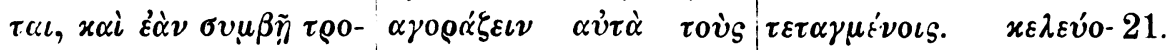

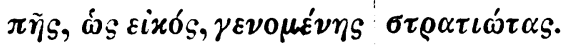

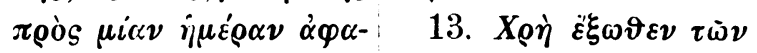

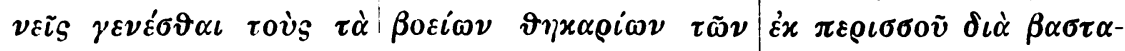

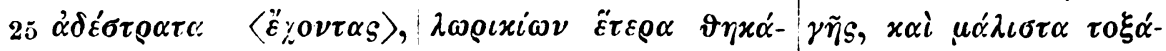

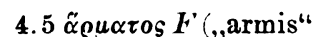
recte Sch.) \| $13 \beta 0 i \omega \nu F$ 14 verhum tantum in volgari usu lictum $\ddot{\alpha} \pi \alpha \xi \lambda \varepsilon \gamma o ́-$ $\mu \varepsilon \nu \circ \nu$ est; derivatur sine dubio a roce. ovis et ab: 17 xоvอб $F \|$ 17. 18

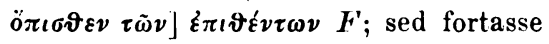
magis conveniet ö $\pi \iota \vartheta \varepsilon v$, quod verbum in codice passim (e. gr. fol. 15 $5^{r}$, II 4 ,

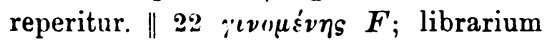

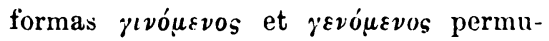
tare lubet || 25 है $\chi 0 \nu \tau \alpha \varsigma$ om. $F$
1. $2 \sigma \alpha \gamma \iota \tau \dot{\omega} \nu A \| 12.13$

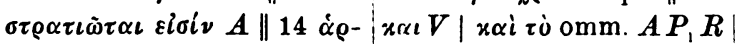

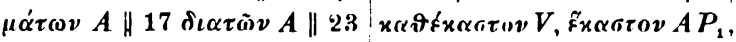

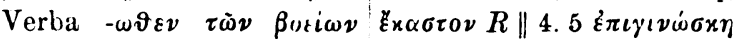

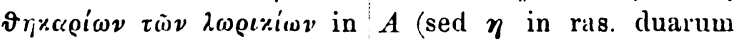
ras. extant in $A$ litterarum), $\varepsilon \pi \iota ; \iota v \omega \sigma x \varepsilon \iota P_{1}$ '

$6 \tilde{\varepsilon} \tau \varepsilon \rho \leftrightarrow i \delta \iota x \dot{\alpha}] \tilde{\varepsilon} \tau \varepsilon \rho \circ \nu \beta \dot{\alpha} \nu-$

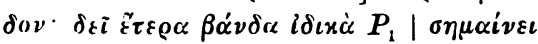

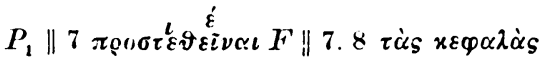
$P_{1}\left\|10 \tau o v \tau \omega v A P_{1} R\right\| 11-14 \mathrm{E}$ verbis

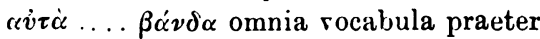

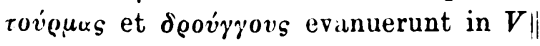

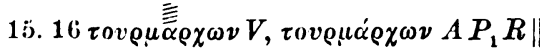
$16 \beta \dot{\nu} \nu \delta \alpha$ inserui $\| 18 \pi \rho o ́ \chi \varepsilon \iota \rho \alpha A P_{1} R$.

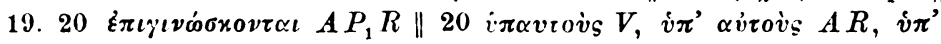

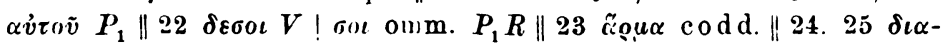
$\beta \alpha \sigma \tau \alpha \eta_{i} ; F V A P_{1}$ 


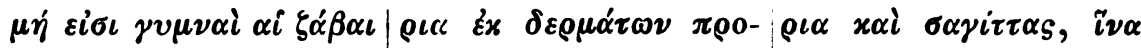

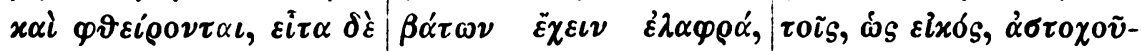

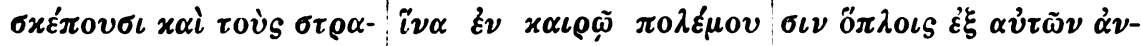

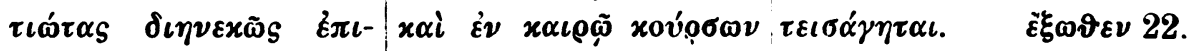

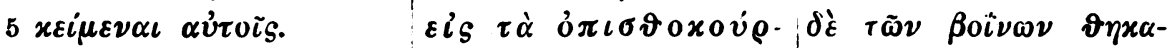

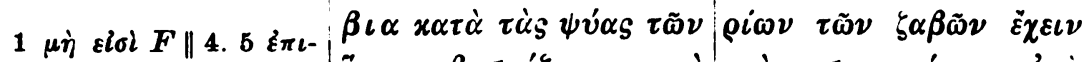
$x \varepsilon\llcorner\mu \dot{\nu} \nu \alpha \iota s$

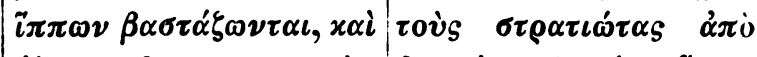

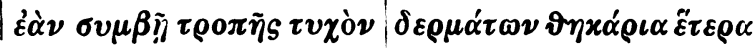

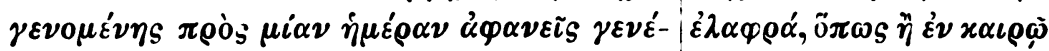

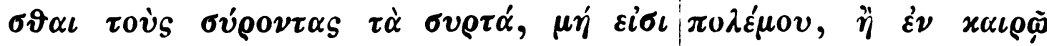

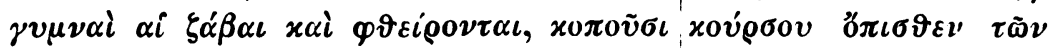

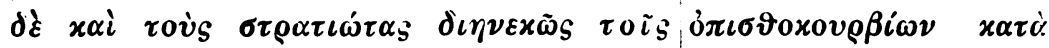

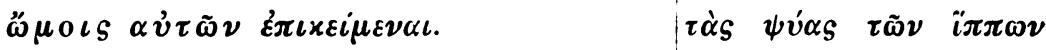

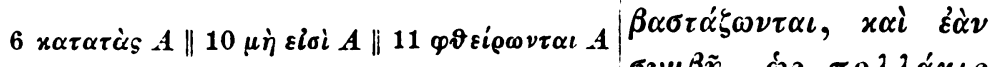
$\sigma v \mu \beta \tilde{\eta}, \dot{\omega}_{S} \pi 0 \lambda \lambda \alpha \dot{\alpha} \iota \mathrm{S}$

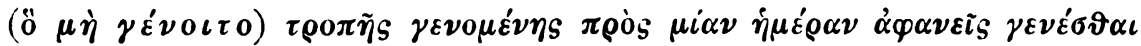

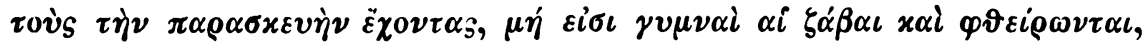

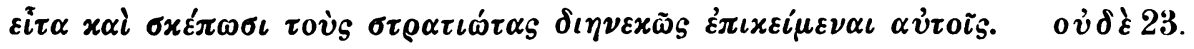

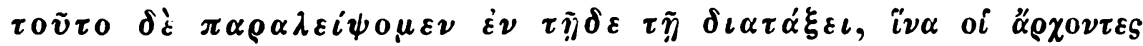

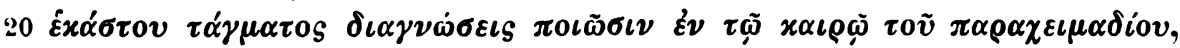

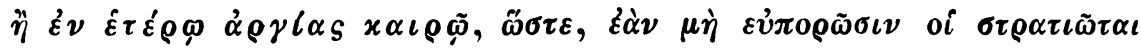

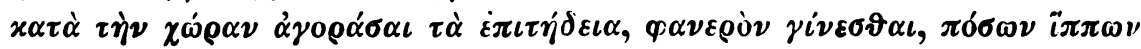

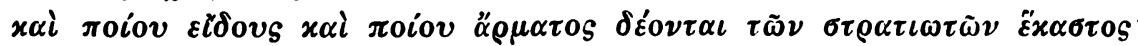

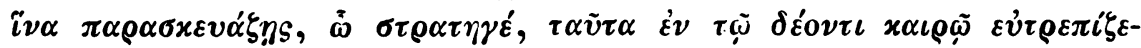

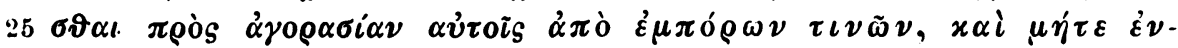

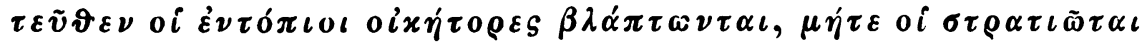

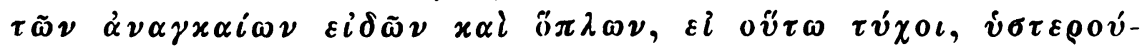

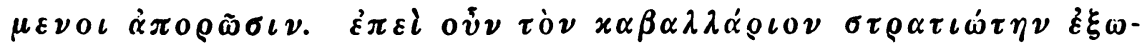

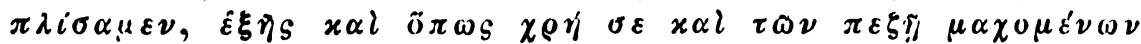

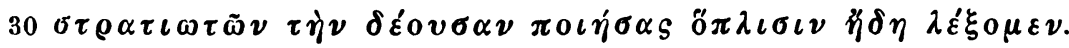

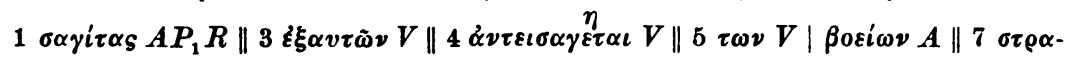

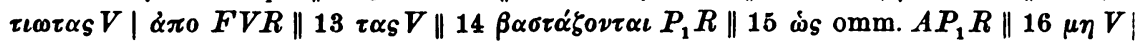

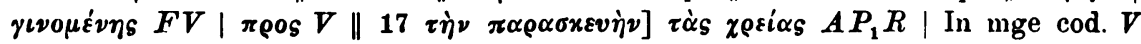

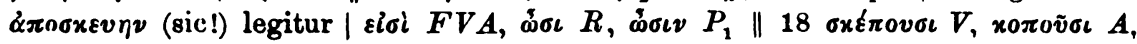

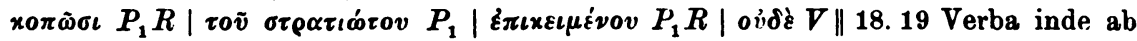

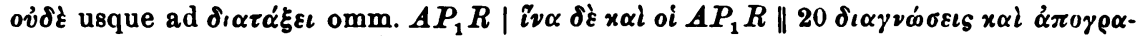

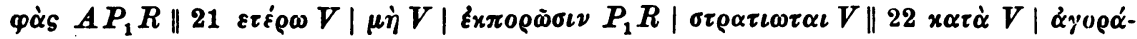

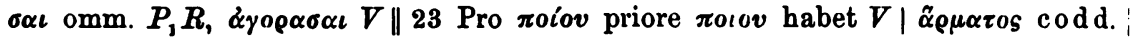

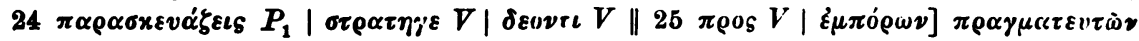

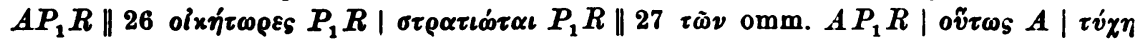

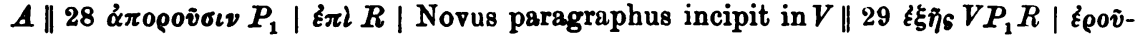

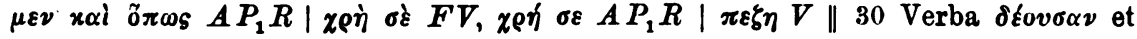

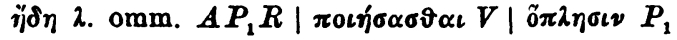


Aus diesen Gegenüberstellungen lassen sich gewisse Folgerungen ableiten. Denn wenn man die Frage aufwirft, ob die Taktik des Kaisers Leo auf die florentinische, oder auf die ambrosianische Fassung der Taktik des sog. Maurikios zurückgeht, so wird man leicht zur Erkenntnis gelangen, daB in chronologischer Beziehung die Taktik Leos früher anzusetzen ist, als die ambrosianische Überarbeitung des sog. Maurikios. Was sich hier scheinbar mit Leo berührt, beruht nicht auf Übernahıe, sondern auf dem Bestreben, die geläufigere und modernere Ausdrucksweise an Stelle der veralteten zu setzen, wobei es nicht ausgeschlossen zu sein braucht, dab so manche Wendungen im ambrosianischen Maurikios auf ein Exemplar zurückgehen, welches durch die Hände Kaiser Leos gegangen ist. Nicht ein neues Werk zu schaffen, schwebte dem Redaktor des ambrosianischen Maurikios vor, sondern nur ein seinen Zeitgenossen leichter verständliches zu geben. Der ganze Wortschatz der ambrosianischen Fassung ist unstreitig jünger als der der Taktik Leos. Doch hierauf kann eines näheren jetzt nicht eingegangen werden; ich muB mich begnügen, das, was sich mir bei Einsicht der Hss als Überzeugung aufgedrängt hat, hier ohne eine eingehende Begründung, die wohl am besten durch die Herausgabe des Textes urkundlich aufgestellt werden kann, einfach den Lesern anheimzustellen.

Anders steht es mit der Frage, die den eigentlichen Kern dieser Ausführungen bildet, in welchem Verhältnisse sich die sog. Konstantinische Taktik zu der des Kaisers Leo befindet. DaB sie mit der Taktik Leos und nicht mit der des sogenannten Maurikios zusammengehört, habe ich schon in meiner akademischen Abhandlung „Bölcs Leo császárnak a hadi taktikáról szóló munkáju“ (Bp. 1898) S. 69-71 wider Franz Salamon unwiderleglich bewiesen. Nun kann aber hier die Frage auftauchen, ob die Konstantinische Taktik nicht eben eine solche Überarbeitung der leoninischen ist, wie die ambrosianische Fassung gegenüber der florentinischen, oder ob die Konstantinische Taktik nicht als ein neues Werk anzusehen ist, etwa in dem Verhältnisse, wie das kriegswissenschaftliche Werk Leos zu dem des sog. Maurikios?

Was die Überlieferung der Konstantinischen Taktik anlangt, so kann sie als eine sehr schlechte gelten. Hier haben wir es mit keiner einzigen älteren $\mathrm{Hs}$ zu tun. Die Editio princeps von dem unermüdlichen Meursius in Jahre 1617 zu Leyden mit Konstantins dazumal bekannten übrigen Werken herausgegeben, beruht auf dem Palat. gr. 393 (saec. XVI) $[p]$, dessen Beschreibung wir bei Wilken Geschichte der Bildung, Beraulung and Vernichtung der alten heidelbergischen Büchersammlungen (Heidelberg 1817) S. 289 und genauer 
bei $\mathrm{H}$. Stevenson senior Codd. mss. Palatini gr. bibl. Vaticanae etc. (Rom 1885) S. 252 finden. Diese Hs birgt ron der Konstantinischen Taktik einen ganz unerheblichen Teil in sich und auch diesen in gestörter Reihenfolge folgendermaßen, wie wir sie mit Hilfe der Ausgabe Lamis (bei Meursii Opera VI 1211-1410) feststellen können:

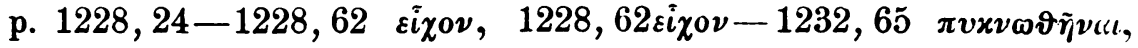

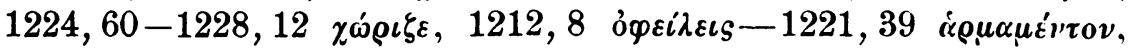

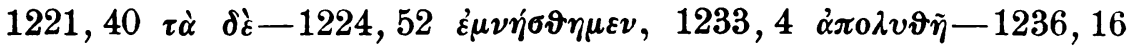

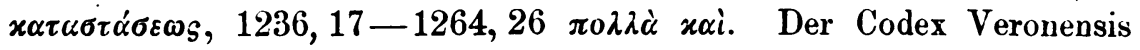
No. 127 (saec. XVI) [v] (s. H. Omont, Les mss. grecs de la bibl. capitulaire etc. de Vérone, Centralbl. f. Bibliothekswesen VIII [1891] S. 492], aus dem Joannes Lami die Tactica Constantini abgedruckt hat, ist wohl vollständiger, gibt aber demungeachtet den Text besonders um die Mitte des Werkes herum in trostloser Verfassung, scheint im übrigen die Taktik ebenfalls nicht vollständig zu enthalten, am Anfange jedenfalls verstümmelt und nicht in der gehörigen Ordnung überliefert. Wenigstens entsprechen der leoninischen Reihenfolge 1221, 40 $-1228,12,1212,8-1221,39,1228,14-1228,62,1229,15-1229,32$, $1228,62-1229,15,1229,33-1233,1$ (dann Ausfall eines beträchtlichen Stïckes) usw. Diese Hs stanmt aus der Sammlung Scipione Maffeis. Die dritte Hs ist der Laurentianus (Ashburnhamianus) No. 1644 (saec. XVI) beschrieben von Rostagno und Festa im Indice dei ('odici greci Laurenziani non compresi nel catalogo del Bandini (Studi Italiani di filologia classica I [1893] 123-232) S. 210. Letztere,

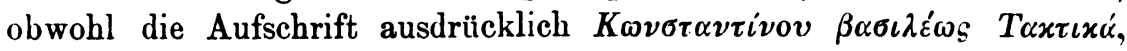

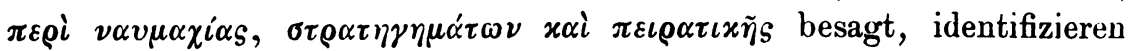
das Bruckstück mit der Taktik Leos (cc. XIX und XX in Meurs. Opp. ed. Lami VI S. 826-904). Gewiß unrichtig; denn wer in Erwägung zieht, daB der Veronensis mit dem, sozusagen, X VIII. Kapitel dor Konstantinischen Taktik schlieBt, und in Betracht zieht den Umstand, daB der Ashburnhamianus früher unter dem Namen Saibantianus 61 resp. 62 bekannt war, auch die Rolle kennt, die die Saibantiani im Bestande der Hss der Kapitularbibliothek zu Verona spielen, wird nicht fehlgehen, wenn er annimmt, daB die Veroneser $\mathrm{Hs}$ und die Florentiner im 16. Jahrhundert ein Ganzes gebildet haben. Was nun $p$ und $v$ anbelangt, so scheinen sie auf Abschriften zurückzugehen, die aus einem Archetypus des 15. oder 16. Jahrhunderts gemeinsam abgeschrieben worden sind.

$\mathrm{Ob}$ nun dieser Archetypus, die vierte Hs, der Cod. Ambros. C 265 inf. (chart. in fol. saec. XVI) ist, aus dessen Abschriften, dem Paris. gr. 2446 und Paris. gr. 3107, Foerster im Hermes (XII, 1877, 


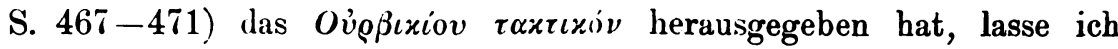
dahingestellt. In dieser $\mathrm{Hs}$ lesen wir nämlich auf ff. 129 Konstantinische Taktik mit der Überschrift: $\beta \iota \beta \lambda i \omega \nu(!) \tau \alpha \varkappa \tau \iota x \delta \nu, \pi \tilde{\omega} s$

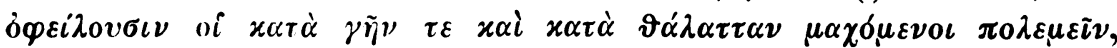

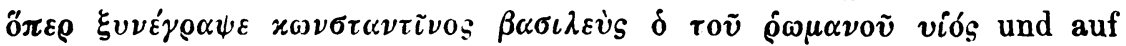

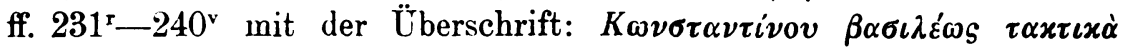

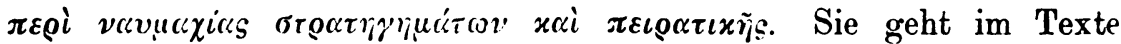
der Naumachica des Basileios Patrikios genau so weit, wie B 119 sup.

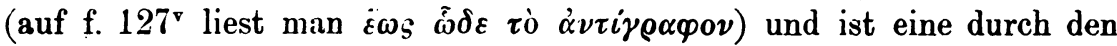
vervollständigten Onasander und die Konstantinische Taktik erweiterte Abschrift derselben.

Um zu $p$ zurückzukehren, muB ich hervorheben, $\mathrm{daB} p$ an willkürlichen Änderungen reicher zu sein scheint, als $v$. Trotz aller Schreibfehler genügen die Lesarten aber, um feststellen zu können, von welcher Art das unter dem Namen Konstantins überlieferte kriegswissenschaftliche Werk ist. Schon der hier folgende Abdruck des den oben mitgeteilten Partien entsprechenden Abschnittes wird uns über diesen Punkt einigermaßen belehren.

Ich bemerke nur, daB auch hier die Zutaten mit gesperrten Lettern gedruckt sind.

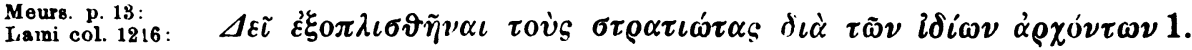

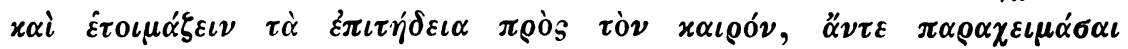

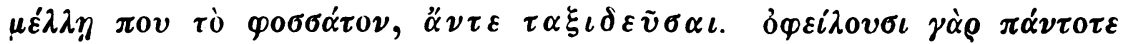

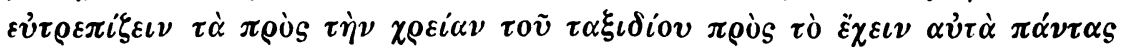

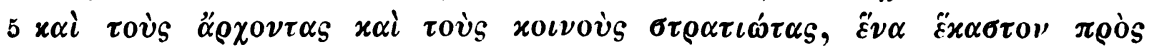

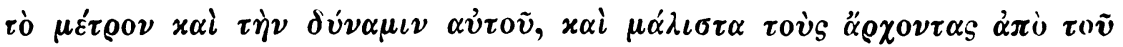

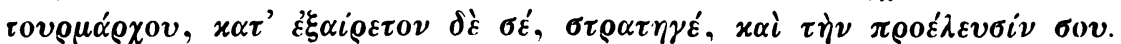

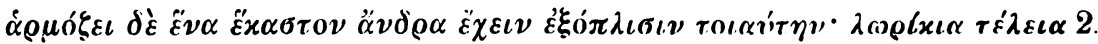

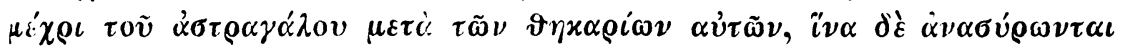

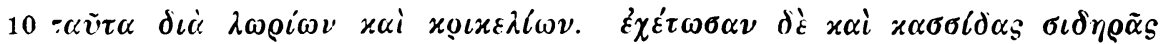

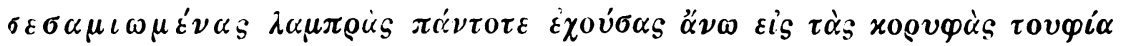

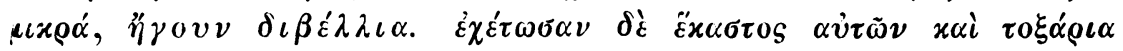

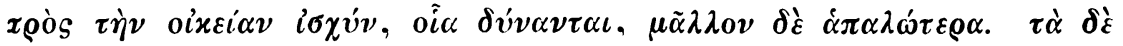

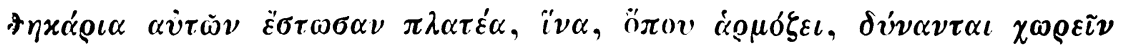

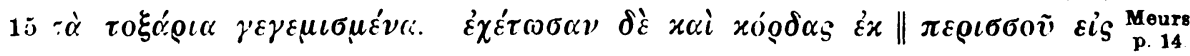

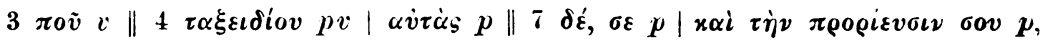

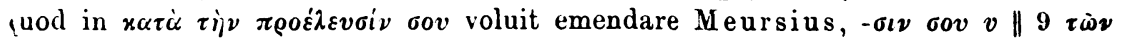

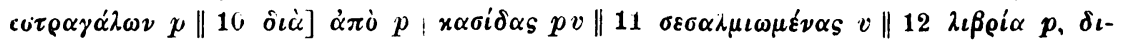

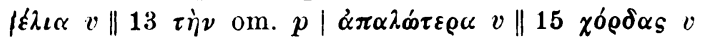




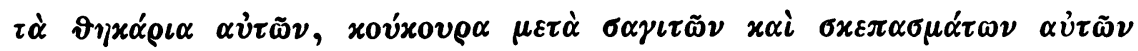

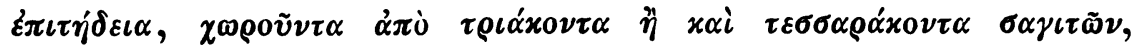

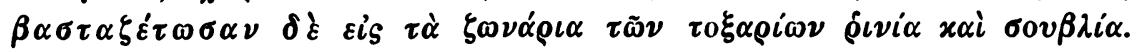

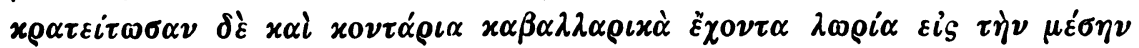

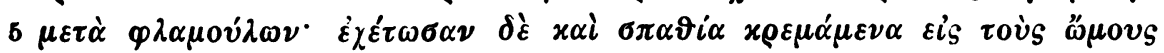

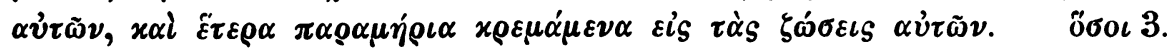

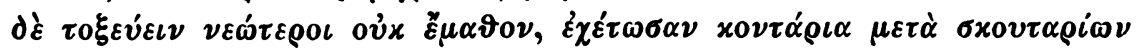

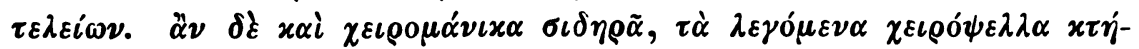

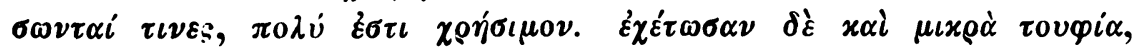

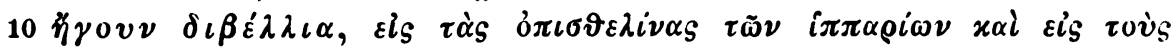

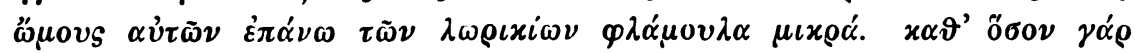

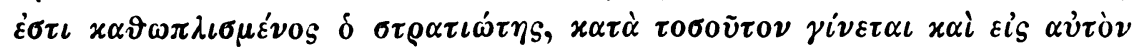

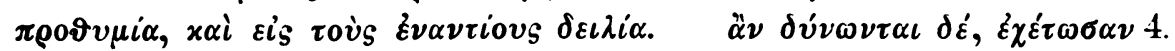

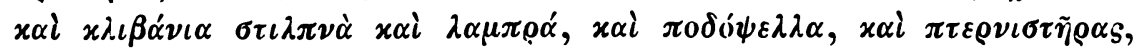

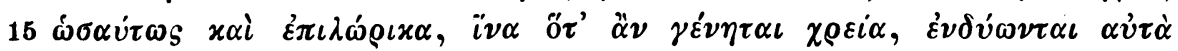

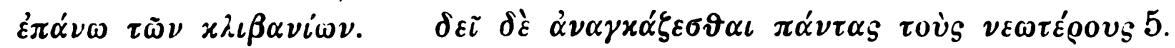

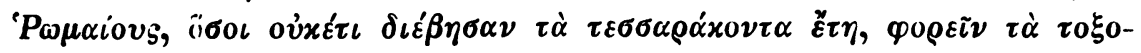

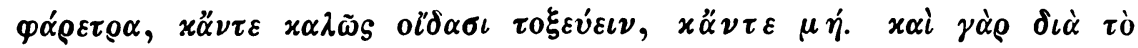

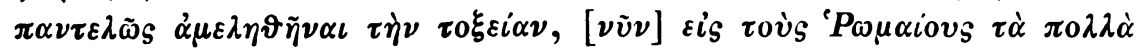

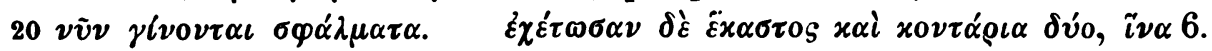

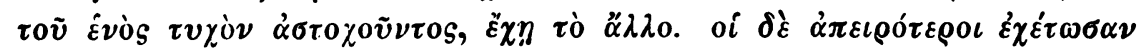

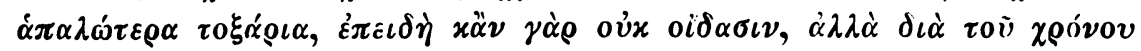

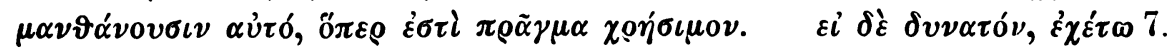

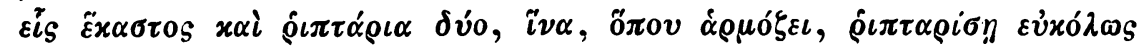

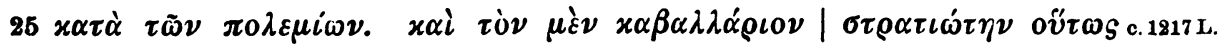

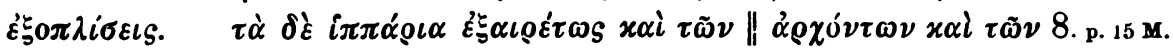

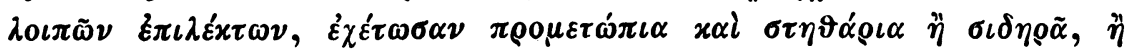

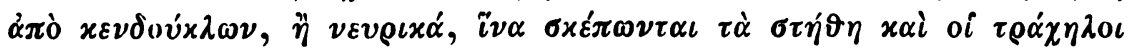

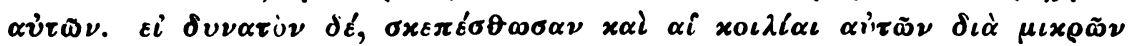

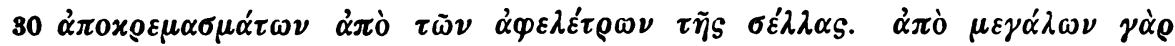

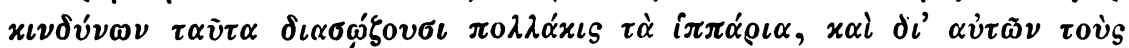

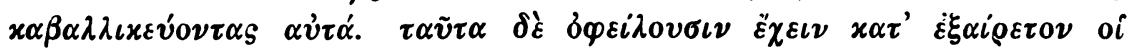

$1 \sigma \alpha \gamma \iota \tau \tilde{\omega} \nu p v$; haec forma, occurrens quoque in codd. $A P_{1} R$, etiam Tacticis

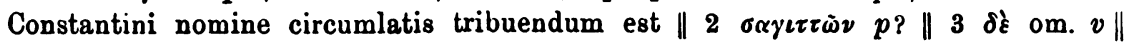

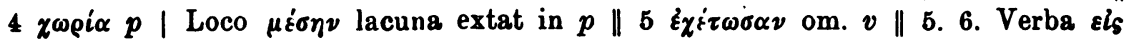

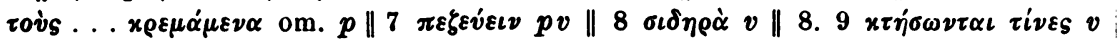

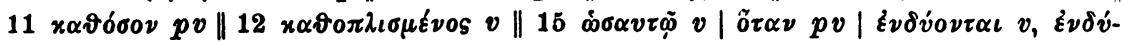

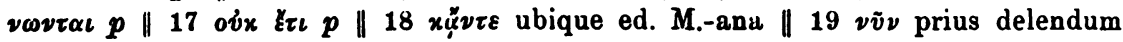

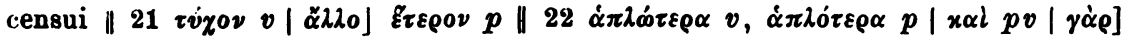

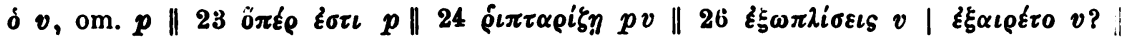

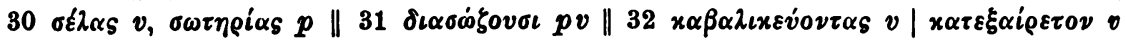




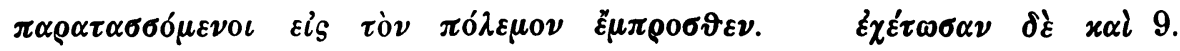

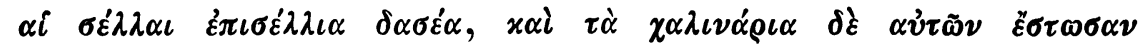

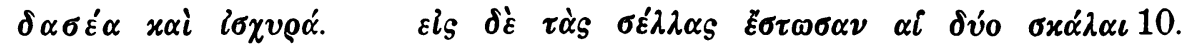

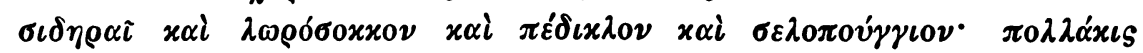

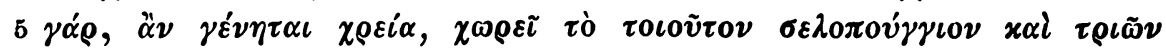

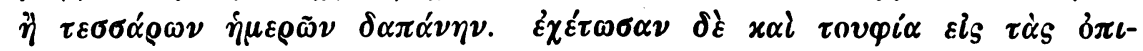

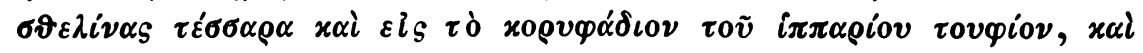

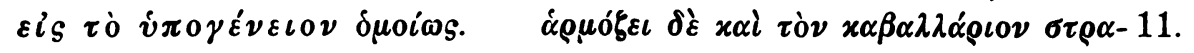

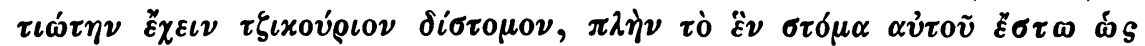

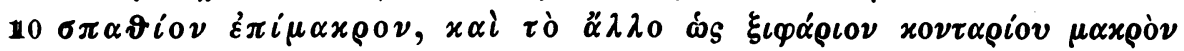

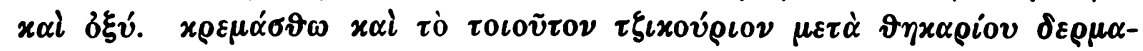

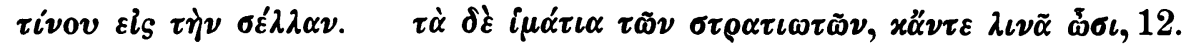

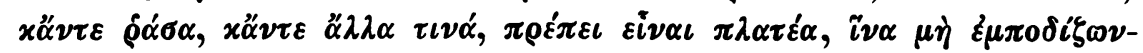

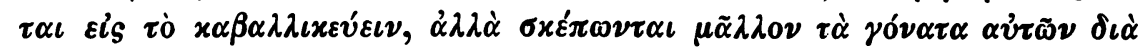

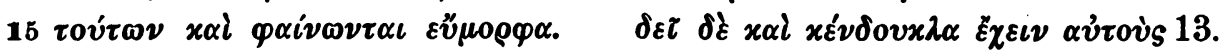

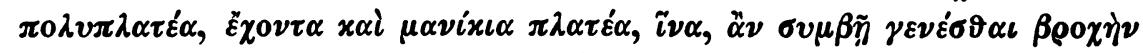

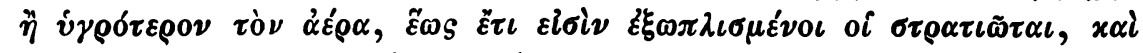

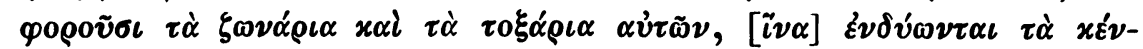

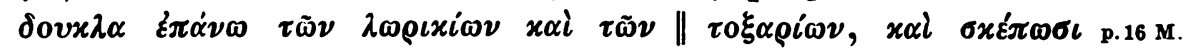

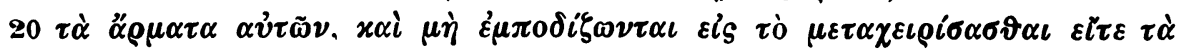

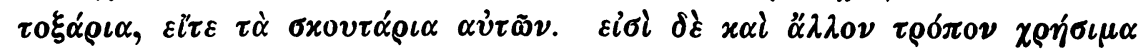

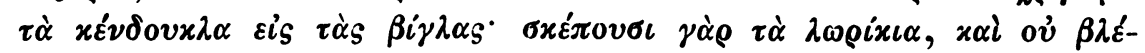

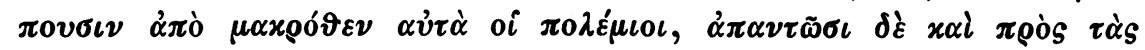

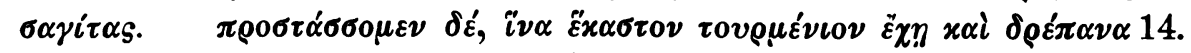

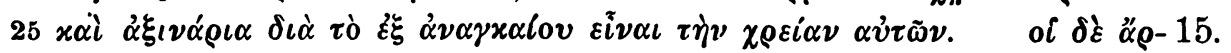

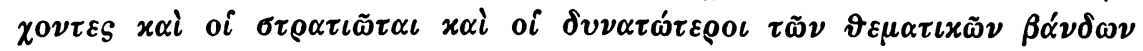

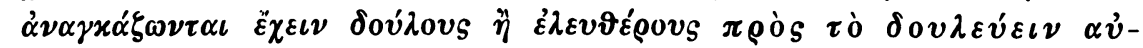

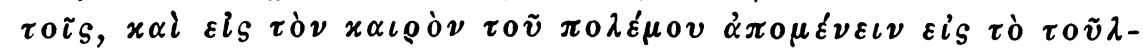

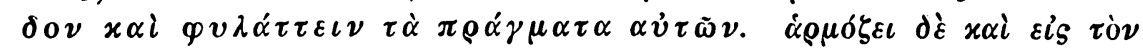

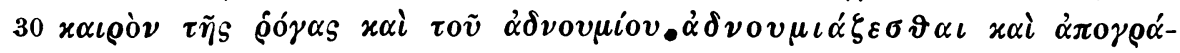

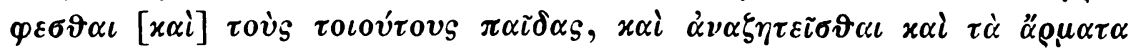

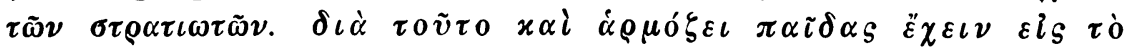

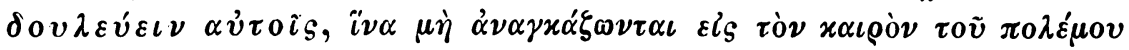

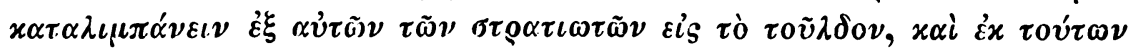

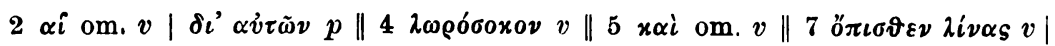

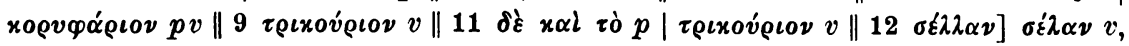

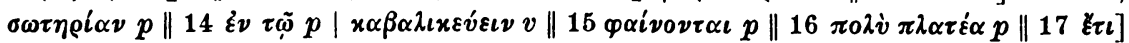

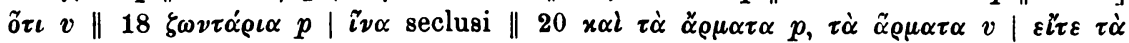

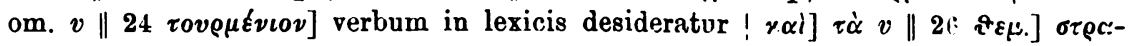

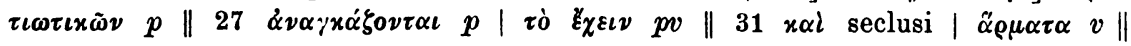

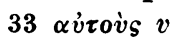

Byzant. Zeitschrift XV 1 u. 2. 


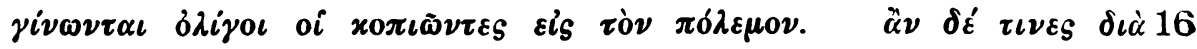

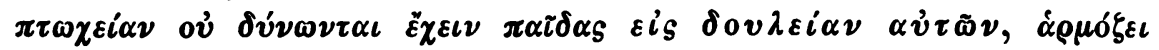

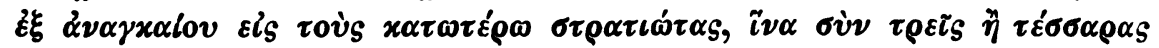

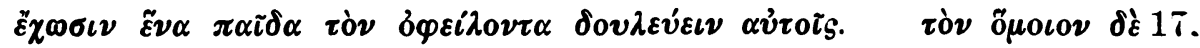

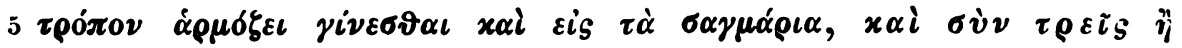

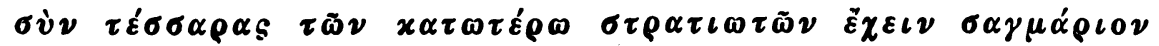

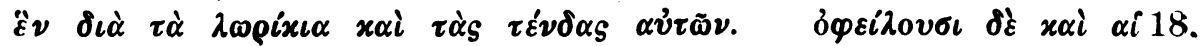

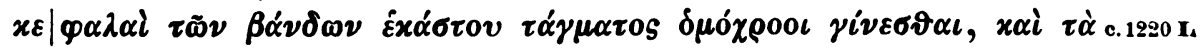

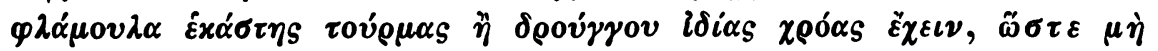

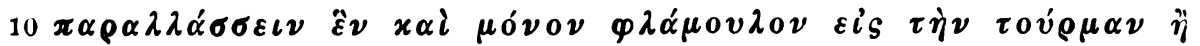

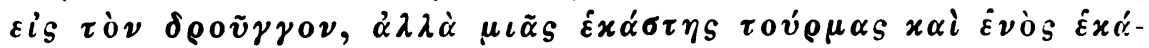

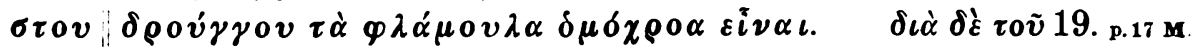

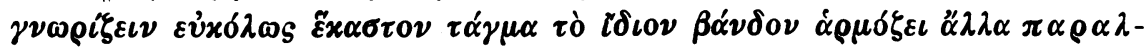

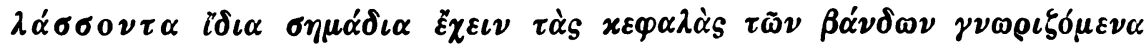

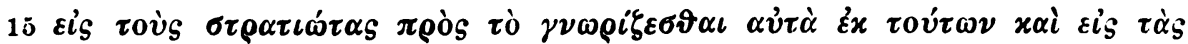

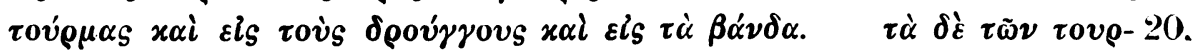

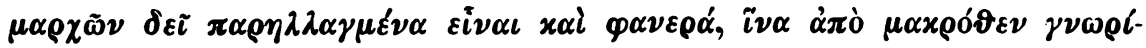

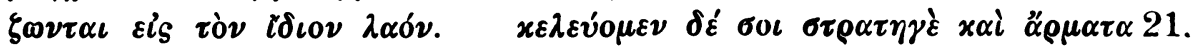

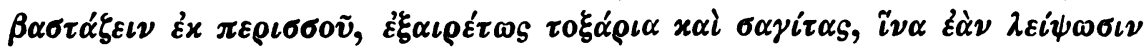

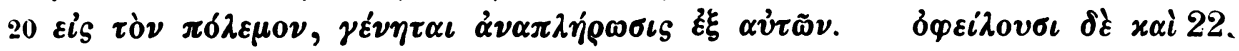

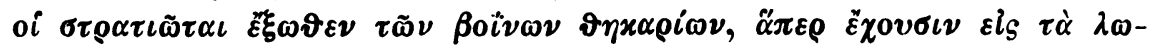

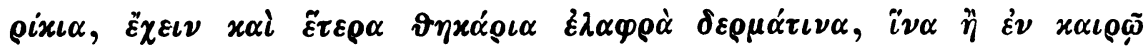

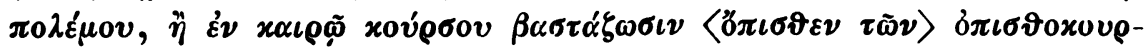

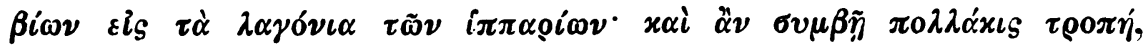

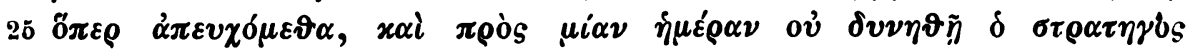

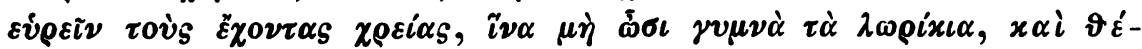

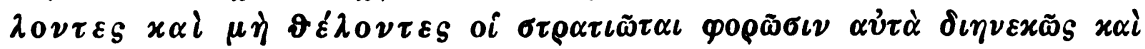

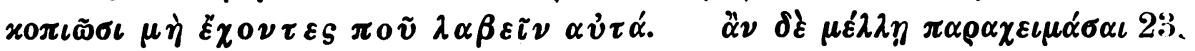

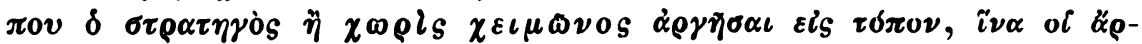

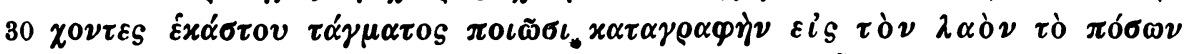

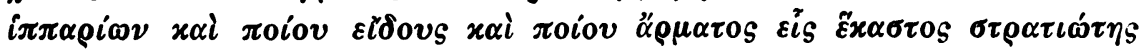

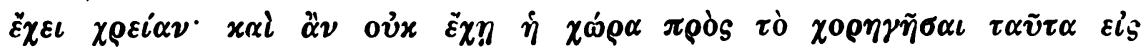

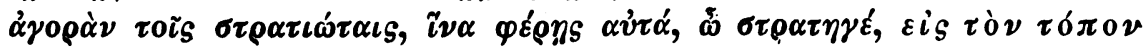

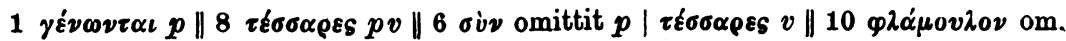

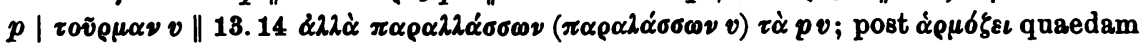

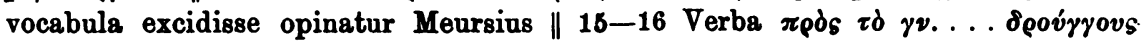

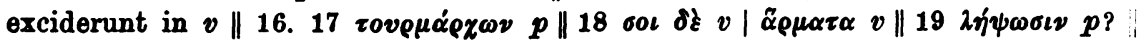

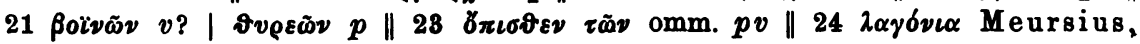

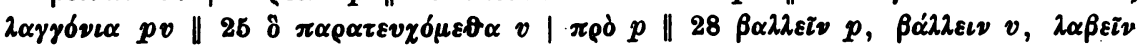

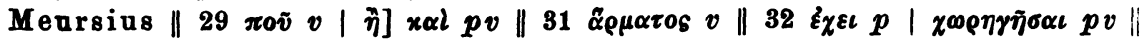
33 ڤँ] ov $v$ 


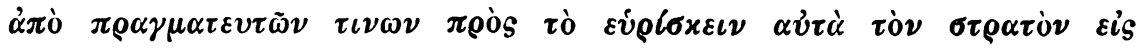

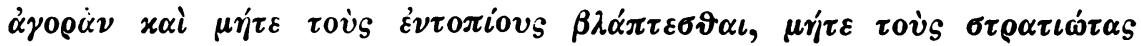

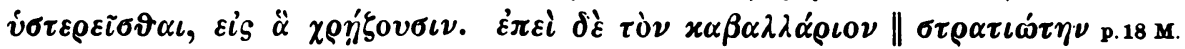

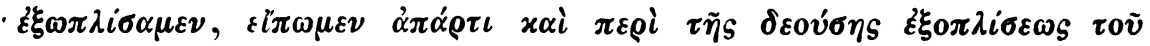

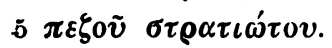

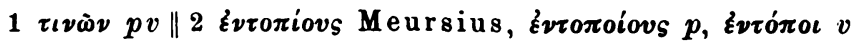

Haben wir nun diesen Text mit dem des Leo verglichen, so werden wir vor allem die Bemerkung machen können, daB er inhaltlich von dem des Leo kaum abweicht, in der Stilisierung aber umsomehr. Jedoch auch in letzterer Beziehung ist die Abweichung nicht so groB, als es den Anschein hat, wenn wir die Fassung $P_{1} R$ vor Augen halten. Zum mindesten ist aber der Unterschied nicht gröBer, als der, der zwischen dem florentinischen Maurikios und dem ambrosianischen obwaltet. Denn auch in unserer Konstantinischen Taktik leuchtet das Bestreben hervor das Leosche Werk den Zeitgenossen ver-

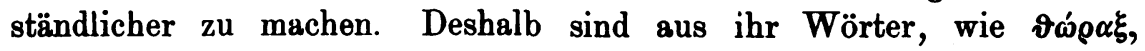

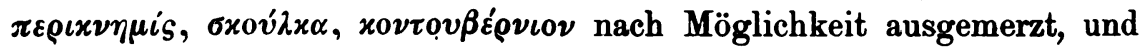

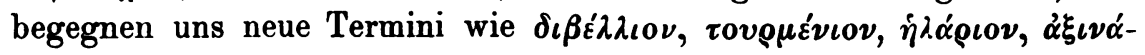
olov usw. Aber aus der Literaturgeschichte hat diese Fassung der Leoschen Taktik zu verschwinden, und es darf ihr nicht mehr Selbständigkeit beigemessen werden, als der ambrosianischen Fassung des sogenaunten Maurikios, die ja auch, was ihre Überschrift anbelangt, unter einem andern Namen geht als die derzeit ursprünglichste florentinische Fassung. Die Konstantinische Taktik ist nichts weiter als eine Etappe der Rezensionsgeschichte der leoninischen, deren ursprünglichster Text in $F V$ vorliegt, zuerst revidierter in $A$, abermals revidierter in $P_{1} R$, zuletzt - allerdings in der Zeit Konstantins VIII überarbeiteter in $p$ und $v$. Ihr Platz ist demnach in einem kritischen Apparate der Taktik Leos, wo sie zum leichteren Verständnis dieses wichtigen kriegswissenschaftlichen Werkes erheblich beiträgt.

II.

Ist nun jedermann nach Prüfung unserer obigen Gegenüberstellungen zu der Einsicht gelangt, daB das Verhältnis zwischen $p v$ und $F V$ der leoninischen Taktik dasselbe ist, wie zwischen der ambrosianischen Fassung des sog. Maurikios und dessen florentinischer, so wird er betreffs der Überschriften zu weiteren Schlüssen zu greifen gezwungen sein. Die Überschriften der leoninischen Taktik (also auch 


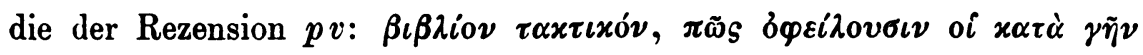

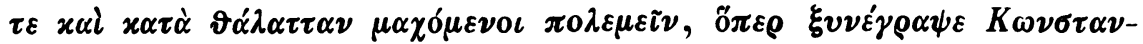

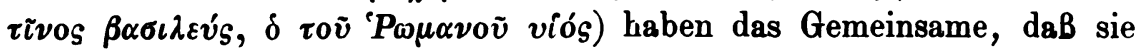
wenigstens nicht ganz zu ignorieren sind. Entweder geben sie den eigentlichen Verfasser an, oder aber sie lassen den mutmaBlichen Zeitpunkt der Überarbeitung durchblicken. Anders steht es mit den Überschriften der Rezensionen der sog. Maurikiosschen Taktik. Hier steht Titel und Werk in scheinbar gar keinem Zusammenhange mit eiuander.

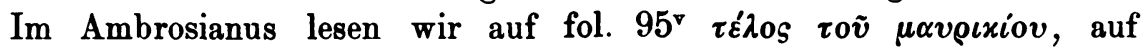

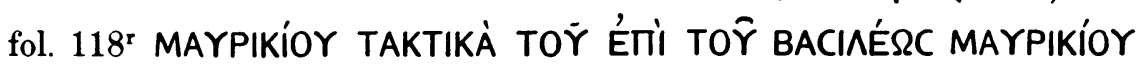
ГETONÓTOC, auf fol. $335^{\circ}$ von derselben Hand einen (wiederholten)

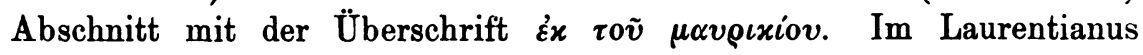
LV 4 hingegen auf fol. $6^{\text {r }}$ ỚPBIKÍOY TAKTIKÀ CTPATHIIKÁ. Und doch gingen bisher alle Bemühungen fehl, die das von Scheffer herausgegebene kriegswissenschaftliche Werk einem Maurikios oder Urbikios zuschreiben wollten. Was den ersteren Namen betrifft, weise ich auf Zachariae v. Lingenthals in dieser Zs. (III [1894] S. 437-457) erschienene Ausführungen (p. 440) hin. Dort lesen wir auch auf S. 441 betreffs der ambrosianischen Notiz, daB „die Notiz den Wert eines historischen Zeugnisses nicht beanspruchen darf, daB sie vielmehr nur das ausspricht, was ein gelehrter Orientale ..... vorschlagen zu können geglaubt hat"....., vielleicht als Bezeichnung der Zeit, wo der spätere Kaiser Mauricius Oberfeldherr war". DaB der Name aber erst nach Leo auftaucht, dafür haben wir zwei Beweise. Erstens den, daB Leo den Verfasser dem Namen nach nicht kennt, es müBte denn sein, daB schon in seiner Zeit beide Namen im Umlauf waren. Zweitens weiB das Scholion, das im Cod. $V$ auf fol. $1^{\mathbf{v}}$ als Quellen der leoninischen Taktik die Werke folgender bezeichnet: $\alpha \rho \rho \iota \alpha \nu 0 \tilde{v}, \alpha \iota \lambda \iota \alpha v$,

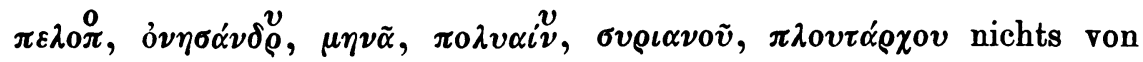
einem Mavoix $0_{:}$, freilich auch nichts von einem Urbikios. Erst im Inhaltsverzeichnisse der Nikephoros Uranosschen Rezension, also in aener, die durch $P_{1} R$ repräsentiert wird (s. meine Ausgabe bei Teubner Incerti script. Byzantini saec. X liber de re militari S. IX) taucht der Name Mavoixios auf, und fehlt der von Minas. Wir sind also nur die ambrosianische Fassung berechtigt unter dem geläufigeren Namen des Maurikios zu zitieren etwa so, wie wir bisher die Rezension $p v$ der Taktik Leos als die Konstantinische Taktik zitiert haben ohne innerliche Berechtigung $z$ war, jber aus Bequemlichkeitsrücksichten. Die florentinische Fassung 
hingegen mit diesem Namen zu benennen, dafür fehlt uns ganz und gar jede Berechtigung!

Sollen wir sie also die Taktik des Urbikios benennen?

Dies führt uns vor allem zur Frage der Zeitbestimmung. Man hat das taktisch-strategische Werk bisher um die Wende des 6. Jahrbunderts entstehen lassen ${ }^{1}$ ) und dafür folgende Gründe ins Treffen geführt: 1) im sogenannten Maurikios stehen im Vordergrunde des allgemeinen Interesses die Perser. Das 2. Kapitel des XI. dóos handelt ganz von ihnen: die Perser stehen bei Aufzählung der Völkerschaften, mit deren Kampfesweise man sich vertraut zu machen habe, an der

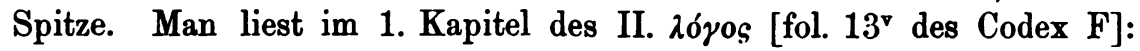

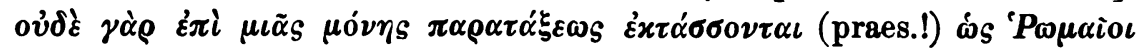

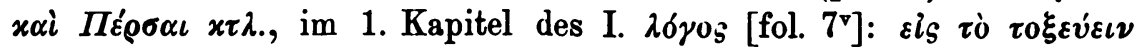

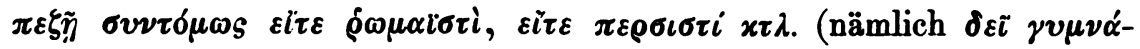

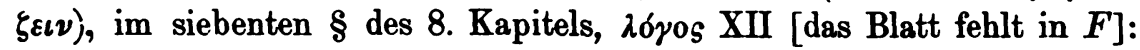

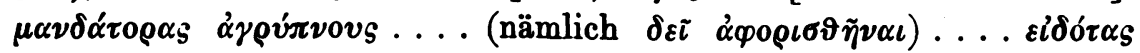

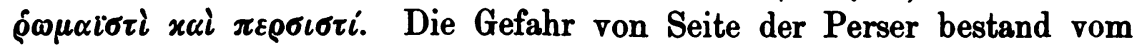
Jahre 626 an bekanntlich nicht mehr; als "terminus ante quem“ ist höchstens das Jahr 637 zu betrachten, in dem Omar dem Reich der Sassaniden ein Ende macht. 2) Von der Mitte des 7. Jahrhunderts an bedeuten die Awaren für die Oströmer keine Gefahr mehr. An ihre Stelle treten seit Konstantinos Pogonatos die Bulgaren. Diese werden bei dem sog. Maurikios nicht als Feinde der Oströmer genannt, jene hingegen als gefährlich angesehen. Kampfesweise und Bewaffnung der Awaren üben einen augenfälligen Einfluß auf die der Oströmer aus.

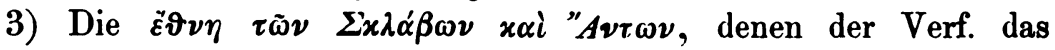

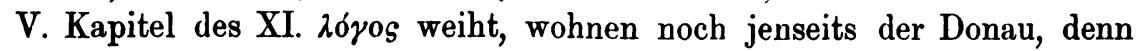

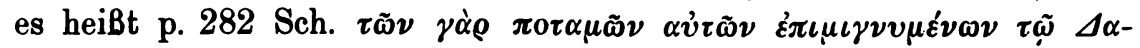

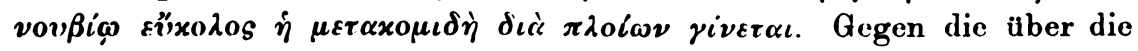
Grenze einbrechenden Slawen ist besonders im Winter vorzugehen sagt der sog. Maurikios p. 278 - denn um diese Zeit sind die Flüsse gefroren und die Wälder zu Schlupfwinkeln ungeeignet; nur darf die gegen sie entsendete Kavallerieabteilung sich nicht nahe bei der Donau aufhalten, sondern einen Tagesmarsch entfernt (Gyomlay S. 11).

4) Die Sprache der Armee zur Zeit des sog. Maurikios war ganz zweifelsohne lateinisch (Gyomlay S. 20), besonders das Kommando.

1) So Zachariae v. Lingenthal a. a. 0., R. Förster, Studien zu den griech. Taktikern (Hermes XII [1877] S. 426-471) S. 458, J. Gyomlay in der gegen mich gerichteten Scnrift Bölcs Leo 'íaktikája mint magyar történeti kútforrás (Budapest 1902, Akad.). 
Der mit dem sog. Maurikios nahezu gleichzeitig lebende, von KoechlyRüstow in den griech. Kriegsschriftstellern (II 2. Leipzig 1855) herausgegebene Anonymus fwar ein Stubentaktiker, der mit seinem Zeitalter in gar keinem Zusammenhange steht.

5) Der sog. Maurikios erwähnt nicht die Bulgaren, obwohl er von

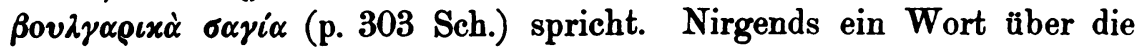
Araber oder Sarazenen! Die Themeneinteilung kennt er nicht, p. 25 liest man wohl in Sch.s-Text $\vartheta \dot{\varepsilon} \mu \alpha$, aber das Wort wird schon von Sch. (S. 400) in $\tau \alpha^{\prime} \gamma \mu \alpha$ emendiert, so wie es bei Leo steht.

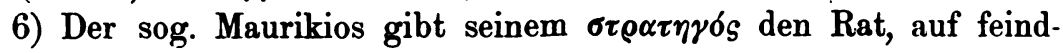
lichem Boden die Lebensmittel und das Brunnenwasser zuerst durch Kriegsgefangene kosten zu lassen. „Denn auch wir haben in den

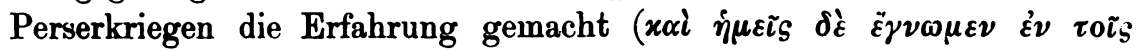
$\Pi \varepsilon \rho \sigma\left(x о \tau_{S}\right)$, daB der Feind selbst die Gerste durch irgend ein Gift verdorben hat" behauptet er S. 221 Sch.

7) Wohl liest man S. 206 Sch., daB es vorkommt, daB außerhalb des befestigten Lagers sich aufhaltende Abteilungen vom Feinde über-

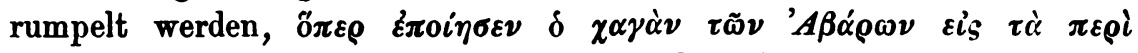

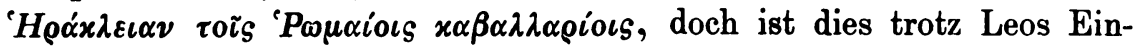

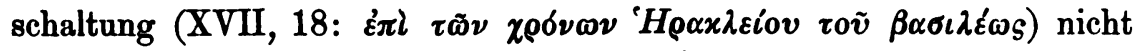
auf das bei Theoph. Chron. (ed. de Boor) 301, 26 u. ff. erzählte, im Jahre 618 geschehene Ereignis zurückzuführen, sondern aller Wahrscheinlichkeit nach auf eine Episode, die sich im Jahre 592 im Feldzuge des Strategen Priskos, ereignet hat. Demnach sind die Tactica Strategica des sog. Maurikios in das letzte Jahrzehnt des 6. Jahrhunderts zu setzen (Förster -S. 458, Gyomlay S. 34).

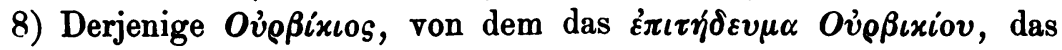
bei Scheffer S. 364-370 steht, herrührt, kann unmöglich der Verfasser sein (Gyomlay S. 36). Dieser spricht nämlich immer in erster Person Sing., jener im Plural. Jener beruft sich an zwei Stellen auf seine

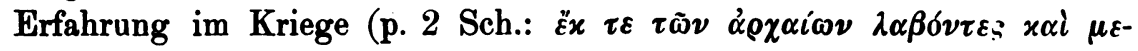

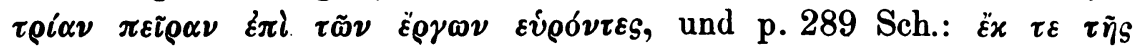

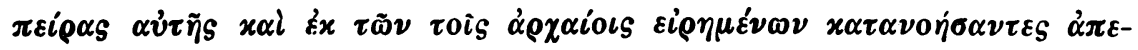

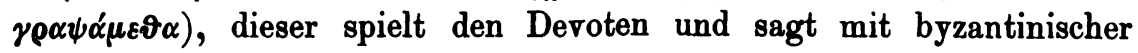

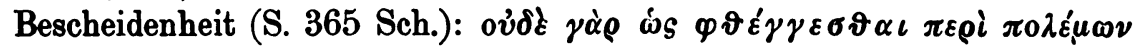
$\varkappa \alpha \tau \alpha \vartheta \alpha \rho \rho \tilde{\omega} \nu \nu . . . \tilde{\omega} \rho \mu \eta \sigma \alpha$ (Gyomlay a. a. 0.). Der Verf. des $\varepsilon \pi \imath \tau \dot{\eta} \delta \varepsilon v \mu \alpha$ lebte unter Anastasios I. (491-518) (vgl. Auct. anonym. de antiquitt.

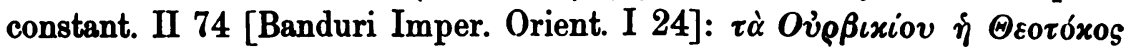

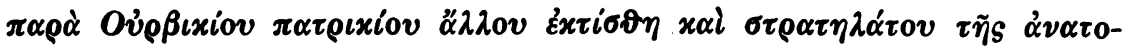

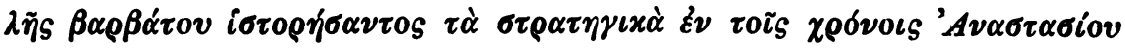

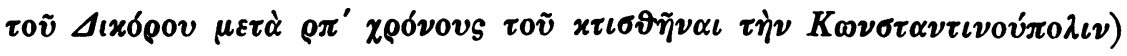


die mehrmalige und zum Teil sehr ausführliche Rücksichtnahme auf Bekriegung solcher Völker, wie Franken, Awaren und Anten paBt auf die Zeit des Kaisers Maurikios, nicht auf die des Urbikios resp. Anastasios (Förster a. a. O. S. 452 u. 457).

Leider verfügt diese ron Zachariae v. Lingenthal, Förster und Gyomlay vertretene Ansicht über kein einziges Argument von zwingender Beweiskraft. Um dies klarzustellen, sei mir gestattet Punkt für Punkt Gegeneinwendungen zu erheben:

1) Es heiBt, daB im Vordergrunde des allgemeinen Interesses beim sog. Maurikios die Perser stehen. Wenn man aber nicht die Reihenfolge in Betracht zieht, in der von den auswärtigen Völkern gehandelt wird, sondern das Ausführliche der Darstellungsweise, so kommen wir zu dem Schlusse, daB die Slawen und Anten ungleich ausführlicher geschildert werden. Das Kapitel über die Perser steht bei Sch. auf S. 254-260, dasjenige über die Slawen auf S. 272-290. In der Taktik des Kaisers Leo rangieren die Sarazenen, um deren willen der Kaiser seine Konstitutionen verfaßt hat (vgl. XVIII 142: $\tilde{\alpha} \pi \alpha \nu \tau \alpha \tau \dot{\alpha}$

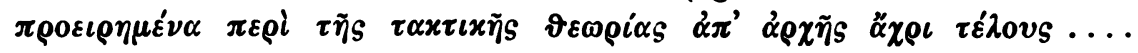

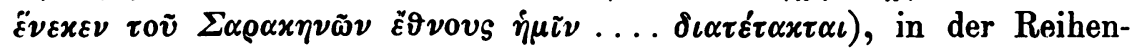
folge der Völkerschaften zuletzt. Zieht man in dieser Hinsicht zwischen den beiden Werken eine Parallele, so gewinnt man den Eindruck, daB bei dem sog. Maurikios die Slawen, bei Leo die Sarazenen im Vorder-

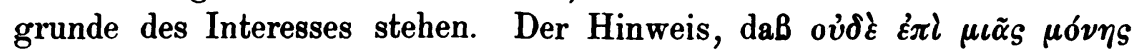

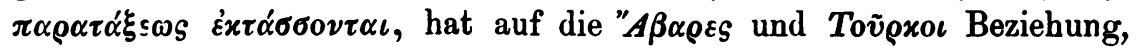
die Aufforderung, man soll die Mannschaft im Pfeilschießen auf römische und persische Art einüben, gibt zu einer Zeitbestimmung gar keinen AnlaB, beweist noch weniger, daB die Perser zur Zeit, als unser Verf. schrieb, Byzanz' gefährlichste Feinde waren. Wohl wird im siebenten §

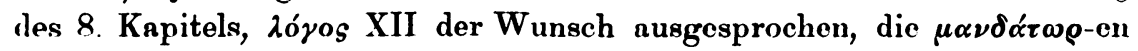
sollen rhomäisch und persisch können, aber in dem Kapitel (I 5, p. 32 Sch.), das die Aufschrift $\pi \tilde{\omega} S \delta \varepsilon \tilde{\imath} \tau o \dot{v} \mathcal{S} \tau \tilde{\omega} \nu \tau \alpha \gamma \mu \alpha \dot{\tau} \tau \omega \nu \not{\alpha} \varrho \chi 0 \nu \tau \alpha_{S}$

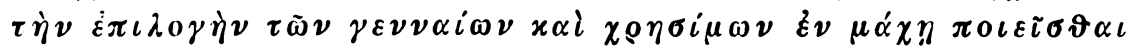

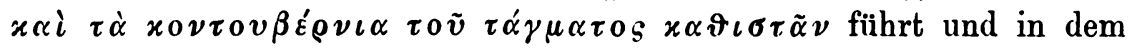

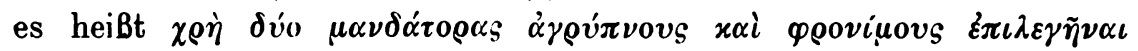

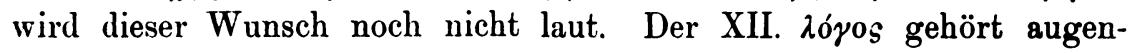
scheinlich nicht zum Werke. Denn erstens schlieBt der XI. גógos, wie wir sehen werden, auf ganz angemessene Art das Buch, zweitens sind

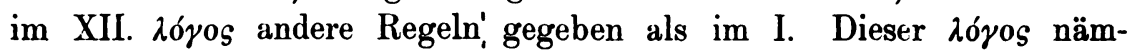
lich und der darauf folgende befassen sich nur mit der berittenen Mannschaft, und erst in XII. licst man $24 \$ \S \pi \varepsilon \rho i \tilde{\eta}_{S} \pi \varepsilon_{\zeta}^{\zeta} \iota x \tilde{\eta}_{S} \tau \alpha^{\prime} \xi \varepsilon \omega_{S}$. Da wird man die Eingangsworte zu diesen Paragraphen leichter ver- 


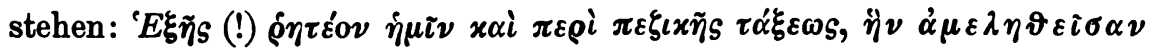

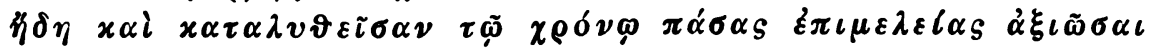

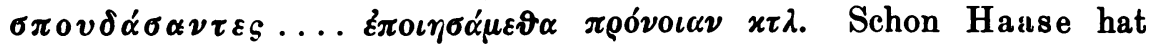
(De militarium scriptorum graec. et lat. omnium edit. instituenda [Bero-

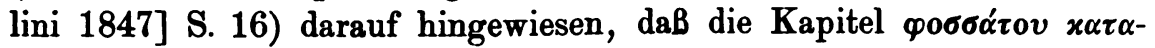

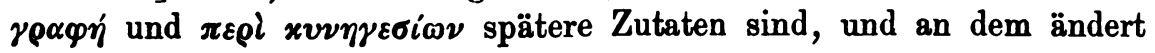
nichts, daB sie der $\pi i \nu \alpha \xi$ fol. $5^{v}$ des Laur. LV 4 aufzählt. Die Kapitel des XII. גójos scheinen aber von demselben Verfasser herzurühren, der die $\varepsilon i \sigma \alpha \gamma \omega \gamma \eta^{\prime}$ verfaBt hat; wenigstens S. 300 lesen wir: $\pi \alpha \rho \alpha \delta i-$

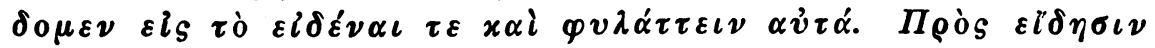

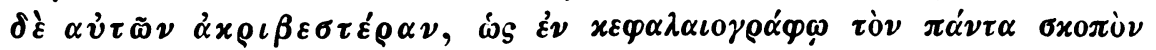

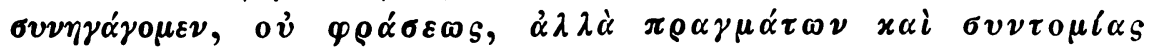

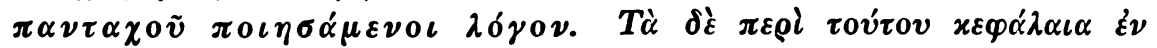

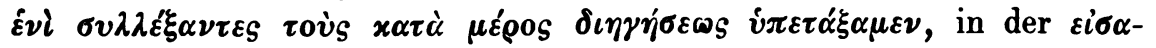

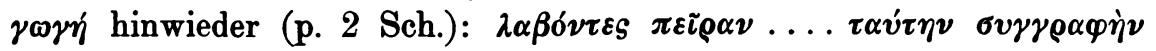

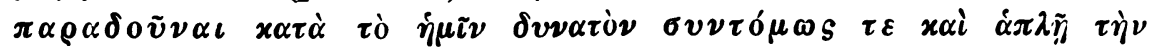

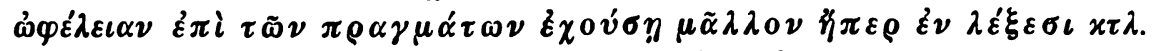

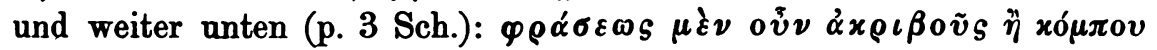

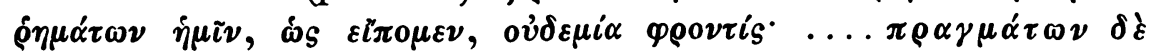

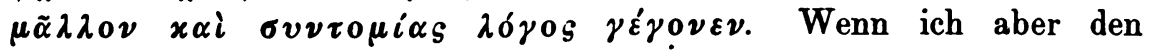

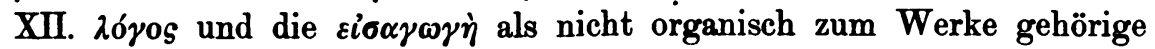
Zutaten betrachte, behaupte ich nicht, daB sie aus viel späterer Zeit sind als das Übrige, in dem gewiB noch manches auszuscheiden sein wird. $\mathrm{DaB}$ die $\mu \alpha \nu \delta \alpha ́ \tau \omega \varrho$-en rhomäisch und persisch können sollen, ist eine sehr harte Anforderung, wenn wir bedenken, daB die Perser das Byzantinerreich nur im Osten bedrängt haben. Aber die Forderung wird begreiflicher, wenn wir voraussetzen, daß der Verfasser unter

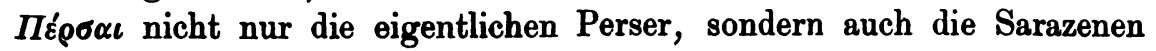
verstanden hat, die das oströmische Reich im Süden, wie im Osten, in Sizilien, wie in Kilikien bedrängt haben. Wir wissen ja aus Krumbachers Lit.-G. ${ }^{2}$ S. 1102, daB „die Hauptschwierigkeit ethnographischer Forschungen in der byzantinischen Geschichte in der fast allen Autoren eigentümlichen Pedanterie liegt, die neuen Völker mit klassischen Namen zu bezeichnen. So heißen z. B. die Türken im höheren Stil Perser, die Russen und andere Slawen Skythen usw." Ich grïnde meine obige Vermutung auf folgende Tatsachen. 1) Nirgends

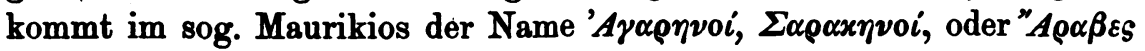

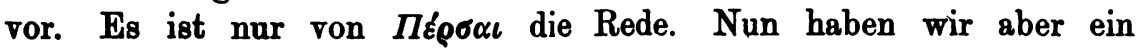
Analogon an Georgios Akropolites. Dieser nennt die eigentlichen Perser

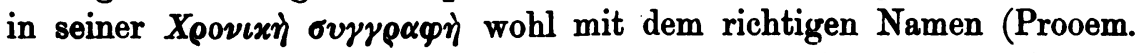
S. 4 ed. Heisenberg), aber die moslimischen Seldschuken ebenfalls mit 
dem Namen $\Pi \varepsilon ́ \rho \sigma \alpha \iota$ (z. B. S. 137). Andererseits haben die Araber selbst dann nicht zu fehlen, wenn wir das Werk gegen die Wende des 6. Jahrhunderts verlegen. Der im 6. Jahrhundert lebende (von KoechlyRüstow) herausgebene Anonymus Byzantinus weiB XL 1 eben so von

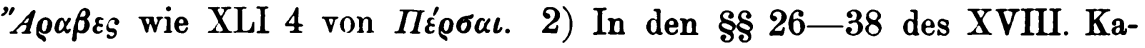
pitels zählt Kaiser Leo einige Schlachtordnungen auf - augenscheinlich gegen die Sarazenen; diese Anordnungen gelten bei dem sog. Maurikios wider die Perser. Leo scheint die Vermutung zu haben, daB seine Vorlage unter den Persern etwas mehr versteht, als es den Anschein hat. Denn obgleich er $\S 38$ von $\tau \dot{\alpha} \tau \tilde{\omega} \nu \Pi_{\varepsilon \rho \sigma \tilde{\nu} \nu} \pi 0 \tau \varepsilon$ spricht,

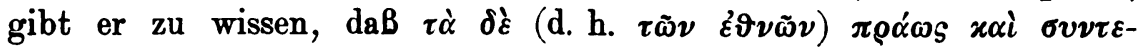

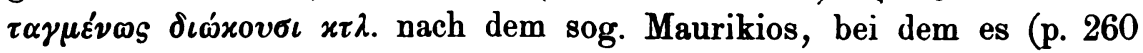

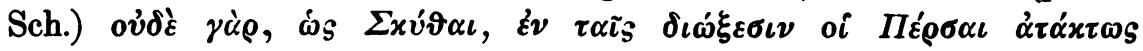

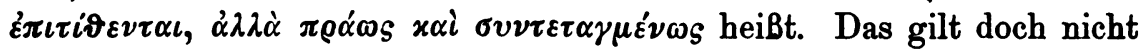

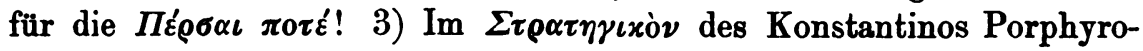
gennetos (herausgeg. bei Meurs. Lami VI, S. 1409-1418) ist der bei dem sog. Maurikios p. 254-260 stehende Abschnitt - der also über die Perser handelt - mit den \$\$ 133-141 der Taktik des Kaisers Leo - die über die Sarazenen handeln - zusammengeschweiBt. Wir werden vielleicht nicht fehlgreifen, wenn wir annehmen, $\mathrm{daB}$ man in

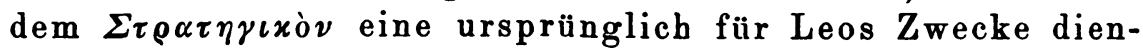
liche Abschrift aus dem sog. Maurikios und einem über die Sarazenen handelnden Feldherrnbericht (vgl. Leo Takt. XVIII

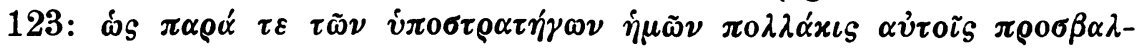

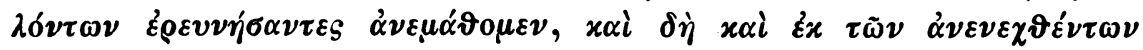

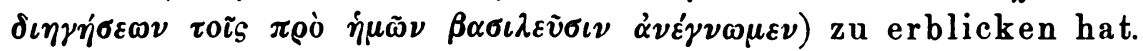

2) Es fragt sich, ob der sog. Maurikios nur solche Völkerschaften ins Auge gefaBt hat, die das Reich ernstlich zu seiner Zeit bedroht

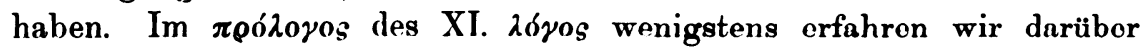

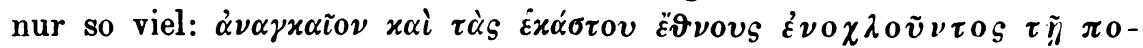

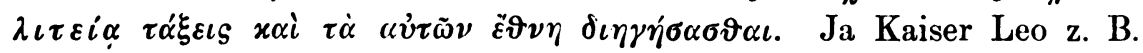
erörtert mit Hilfe seines Gewährsmannes die Kampfesweise der Toṽoxoเ (=Ungarn) und macht uns mit ihrem ethnischen Charakter vertraut,

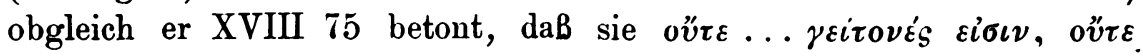

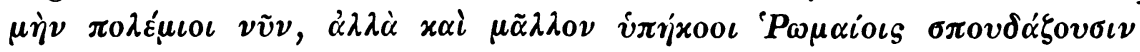
$\dot{\alpha} v \alpha \delta \varepsilon i x v v \sigma \vartheta \alpha \iota$. Die Bulgaren werden bei dem sog. Maurikios nicht als Feinde der Oströmer genannt. Leo übergeht sie unter dem Vor-

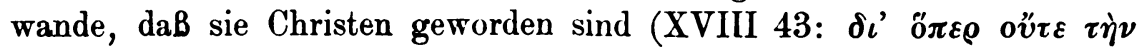

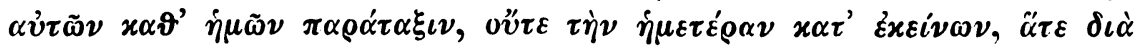

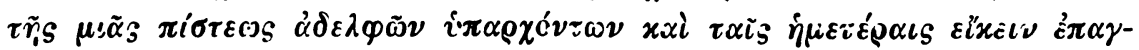

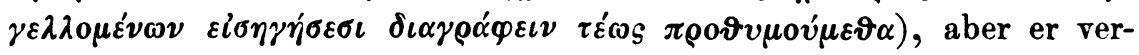




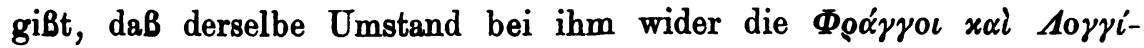

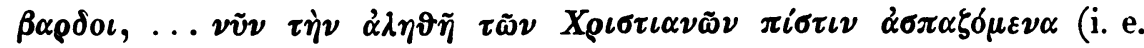
है v $\eta$, XIII 77) gar keinerlei Geltung hat. Natürlich, hier flossen ihm Quellen zu, dort keine. Derselbe Grund kann auch bei unserem sog. Maurikios vorgewaltet haben. Er konnte möglicherweise über die Bulgaren nicht so viel erfahren haben, um daraus ein Kapitel zu machen. Nirgends wird expressis verbis angegeben, daB die Awaren zur Zeit des Verfassers das Reich gefährdet haben. Man kann aus unserer Schrift nur so viel herauslesen, daB sie es zu beunruhigen pflegen. Und das ist auch nach Kaiser Maurikios geschehen, um nur das Jahr $677 \mathrm{zu}$ nennen. Ihre Kampfesweise und Bewaffnung üben einen augenfälligen EinfluB auf die rhomäische aus! Zugegeben, aber ein EinfluB dieser Art betätigt sich nicht von heute auf morgen. Nur wiederholte Bekanntschaft mit der Kumpfesweise eines feindlichen Volkes konnte die Byzantiner, die besonders in der Taktik (weniger auf dem Gebiete der Chargenbenennungen und der Bewaffnung) sich äuBerst konservativ zeigen, zu Neuerungen bewogen haben.

3) Ich lese aus dem Kapitel über die Slawen nicht heraus, daB sie noch jenseits der Donau wohnen. Ich lese etwas mehr heraus. S. 272 Sch. beginnt unser Kapitel mit den Worten: Tü $\ddot{\varepsilon} \vartheta \nu \eta \tau \tilde{\omega} \nu$

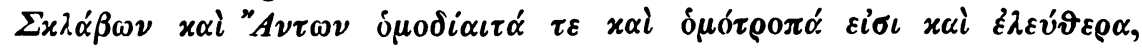

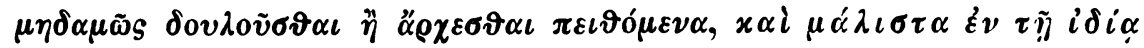
$\chi \dot{\omega} \rho \alpha x \tau \lambda$. Gyomlay liest S. 11 aus diesen Sätzen heraus, daB sie schon seit geraumer Zeit auf die Ufergebiete diesseits der Donau ihre „Einfälle“ gemacht haben. Kaiser Leo gibt diese Stelle (XVIII 98)

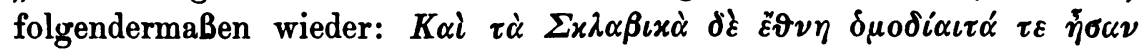

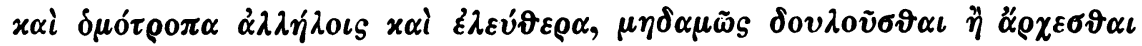

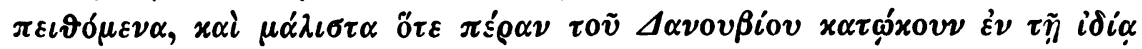
$\chi \omega ́ \rho \alpha$. Nach Leo ist es sicher, daB sio nicht mehr jenseits der Donau ihre Wohnsitze haben (d. h. im heutigen Rumänien), nach dem sog. Maurikios, daB sie wohl jenseits der Donau wohnen, aber auch diesseits des Stromes nicht vollständig zu bezwingen sind. Das kann noch immer für die Zeit vor Justinianos II gelten (s. Gelzer bei Krumbacher GBL.8 S. 956) und gut. nach Herakleios. Ja, es können auch jene Slawen mitinbegriffen sein, die nach dem persischen Schriftsteller Gurdêzî (zuerst herausgegeben vom Gfen Géza Kuun Magyar Honfoglalás kútföi [Budapest 1900] S. 150-194) S. 178 ff., S. 168 ff., als Nachbarn der Magyaren und ron diesen oft hart bedrängt geschildert werden.

4) Das Kommando in der rhomäischen Armee war zur Zeit der Abfassung unseres Werkes zweifelsohne lateinisch. Das zeigen die 
Kommandowörter, die im sog. Maurikios im III. und XII. dó yos reichlich eingestreut sind, von Leo noch einigermaßen verstanden und interpretiert werden, dem Abschreiber des Laur. LV 4 aber geradezu spanische Dörfer sind. Es ist selbstrerständlich, daB in einem so ausgedehnten Heerwesen, wie das byzantinische, die althergebrachte Kommandosprache nicht so rasch über den Haufen geworfen werden konnte. Längst konnte die Armeesprache schon griechisch sein (wozu hätte denn auch sonst unser Verf. sein ganz ausnehmend praktisches Handbuch in griechischer Sprache geschrieben) und mit den Kommandowörtern blieb es beim Alten. Was den "Stuben-Taktiker" Koechlys anbelangt, sei mir gestattet aus der Vorrede Koechlys und Rüstows folgende drei Stellen anzuführen: (II b. S. 39) „Endlich noch ein Wort über die Sprache. Schon in der Einleitung ist S. 101 angedeutet worden, daB in dieser Beziehung unser Anonymus alles Lob verdient, daB er im Verhältnis zu seiner Zeit und der einreißenden Korruption noch ein recht reines Griechisch schreibt." (II b S. 38) „Von der Person des Verfassers wissen wir allerdings nichts: aus der Berufung auf eigene Erfahrung aber XIX 23, aus der ausführlichen und gründlichen Behandlung des ganzen Kapitels über die FluBübergänge mit besonderer Hinweisung auf die Donau, wozu namentlich auch die Darstellung und Kritik der fliegenden Fähre des Apollodoros gehört XIX 7-14, möchte man schließen, daB er als Sachverständiger und zwar als Ingenieur einem oder mehreren Feldzügen an der Donau beigewohnt hat.“ (II a. S. 101) „Mit einem Worte, wir könnten sehr zufrieden sein, wenn Asklepiodotos und Aelianus ihre Zeit so lebendig und frisch berücksichtigten, wie unser Byzantiner die seine!"

5) Die Themeneinteilung kennt Maurikios nicht. Leo kennt sie (z. B. XVIII 139. 153. 154. 155. 156, also in Partien, die nicht aus unserem Maurikios entlehnt sind, also keine Beweiskraft haben). Von Belang ist aber nur eine Stelle bei Leo IV 8: oi $\mu \alpha \iota \delta \dot{\varepsilon}$, $\boldsymbol{\omega}_{S}$ oi $\pi \alpha-$

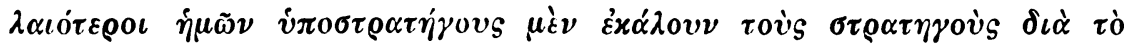

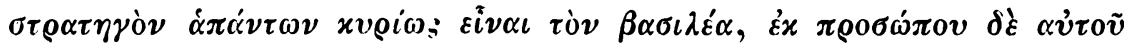

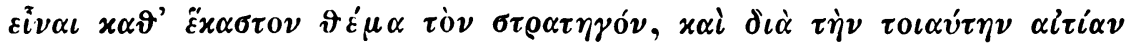

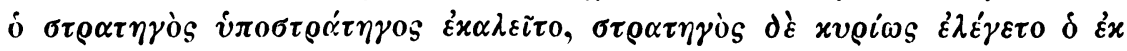

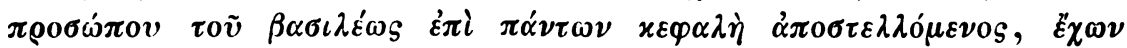

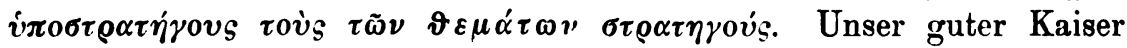
meint also, daß die $\pi \alpha \lambda \alpha \iota$ ó $\tau \varepsilon o \_\iota$ die Strategen (also Präfekte der $\vartheta \varepsilon^{\prime} \mu \alpha \tau \alpha$ ) wohl Hypostrategen nannten, deun als eigentlicher Oberfeldherr galt ihnen der Kaiser, da aber in einem jeden Thema seine Person der Stratege vertrat, sc hieB der Stratage [in seinem Sinre] Hypostrategos, Strategos im eigentlichen Sinne aber der, der in Vertretung des Kaisers 
an die Spitze aller gestellt wurde und zu Hypostrategen die Strategen der Themata hatte. Das ist die Meinung Leos, der vorhergehend den Begriff des Strategos und des Hypostrategos genau so definiert, wie der sog. Maurikios. Nun behauptet Gyomlay S. 19, daB die Ansicht Leos einfach irrig ist; die vorherakleiossche Zeit kennt keine Themenverfassung. Ja, hingegen wie dann, wenn das sog. Maurikiossche Werk denn doch nicht so weit zurückdatiert werden muß? Es lassen sich aber überdies zwei Möglichkeiten denken 1) es war für den Verf. kein zwingender Grund vorhanden seine Taktik und seine strategischen Regeln im Hinblick auf die Themeneinteilung zu verfassen. Leo, der doch das ganze Werk in seine Taktik eingeschmolzen hat, konnte ganz gut die alten Termini gebrauchen, die Schlachtordnungen des sog. Maurikios wieder auftischen, über TroB und Lagerstellung längst Vorgebrachtes wieder vorbringen, usw., ohne gezwungen zu sein, die Themenordnung in diesen Stücken - von zwei Stellen abgesehen öfter zu berühren. Und doch ist sein Buch ein offizielles Handbuch, ein ausführlicher Leitfaden, der so ziemlich alle kriegswissenschaftlichen

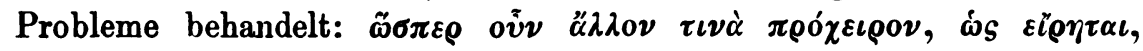

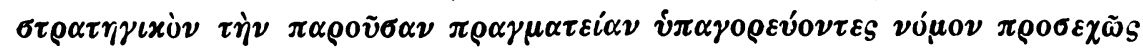

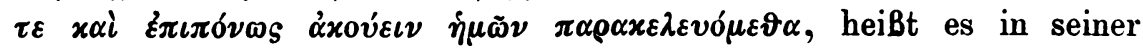
Vorrede \& 8. Dagegen ist das sog. Maurikiossche Werk nur ein praktisches Handbuch voll bescheidener Ratschläge. 2) Man muß mit der auch in unserem modernen Zeitalter beobachteten Tatsache rechnen, da $B$ Kenntnisse organisatorischer Einrichtungen der großen Masse immer mangeln werden. Stil, Ausdrucks- und Kompositionsweise, Gedankengang in unserem sog. Maurikios zeigen genugsam, daB unser Mann wohl eine praktische Soldatennatur ist, aber auch in jeder Beziehung "humi repit". Solche Leute halten fest an althergebrachten Ausdrücken.

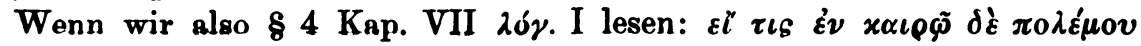

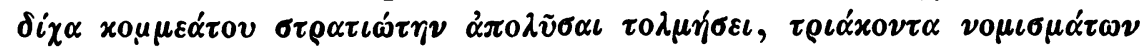

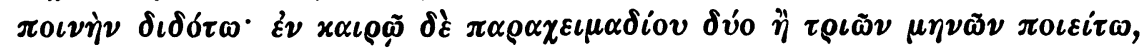

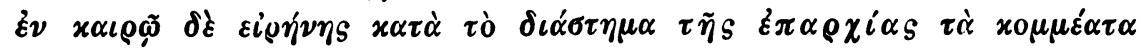

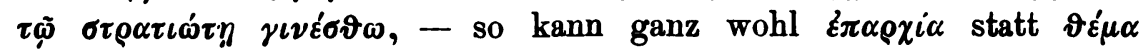
stehen, wie denn auch die ambrosianische Fassung (fol. 126 $6^{\nabla}$ ) $\tau \tilde{\eta} s$

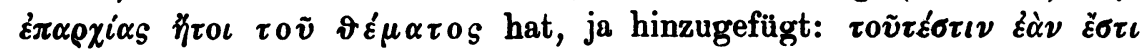

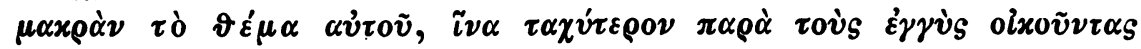
d $\dot{\pi} 0 \sigma \tau \varepsilon \dot{\varepsilon} \lambda \lambda \eta \tau \alpha \iota$. Kaiser Leo aber begnügt sich mit dem Worte $\dot{\varepsilon} \pi \alpha \rho \chi^{i \alpha} \alpha$ (VIII 15); die Rezensionen $F V$ und $A$ bieten es, die Rezension $P_{1} R$ und demzufolge auch die von $p v$ schalten es aus dem Texte aus. Das

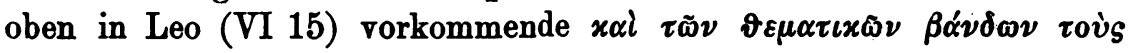

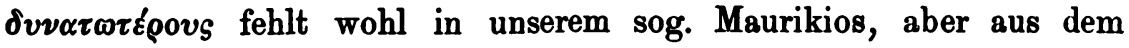


Fehlen dieser Stelle ist für ihn kein Schluß zu ziehen, nur für Leo, der aber trotz allem die Themeneinteilung verhältnismäBig an sehr wenig Stellen berührt, viel weniger häufig, als z. B. der Verf. der Schrift De velitatione bellica.

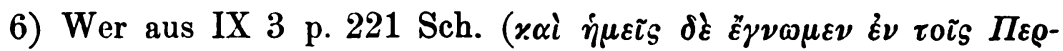
бixois) auf selbst Erlebtes des Verf.s schließen möchte (wie auch Förster a. a. O. S. 458) geht denn doch zu weit. Ja, wenn es in erster Person behauptet worden wäre! Das ist nur so viel wert, als wenn wir in der Rez. $p v$ (ed. Meurs. Lami p. 1264), die doch in solchen Dingen mit einer gewissen Selbständigkeit verfährt, folgendes

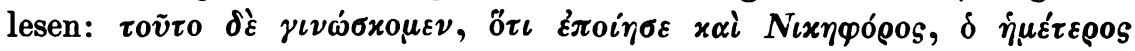

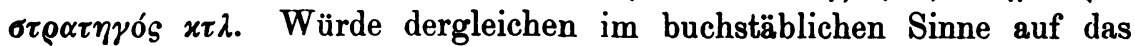
Ich des Verf.s bezogen werden, so hätte der Überarbeiter sich gehütet

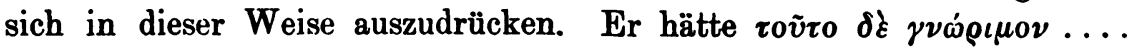

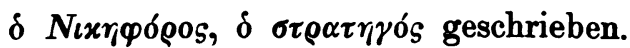

7) Es ist kein Grund dafür vorhanden, daB man das Zeugnis Leos (VII 18) zu verwerfen habe. Wenn Leo historische Tatsachen anführt (was wohl selten geschieht) z. B. IX 13, XI 25, 26, gibt er nichts Irriges. Weshalb sollen wir also QuellenmäBiges verwerfen? Psychologisch ist es doch erklärlicher, wenn der Kompilator, der sich noch dazu ziemlich peinlich an seine Vorlage hält, Neues - um MiBverständissen vorzubeugen - einschaltet, als ohne erheblichen Grund etwas, was er nicht ganz sicher weiß. Nicht von seiner Ansicht, Meinung, Auffassung ist hier die Rede, sondern von einer chronologischen Fixierung.

8) Zur Frage der Überlieferung des Namens Oủ@ßixios übergehend müssen wir etwas weiter ausholen. Im Cod. Ambr. B. 119 sup. (A) - und auch in jüngeren Hss - sind mehrere Epigramme auf uns gekommen, die auf die Sammlung der kriegswissenschaftlichen Werke unstreitig Bezug haben, teilweise von Förster (a. a. O. S. 462) herausgegeben worden sind, und deren zwei man auch in der griechischen Anthologie finden wird. Von diesen Epigrammen stehen auf fol. $96^{\mathrm{v}}$ drei vor dem von Förster (a. a. 0. S. 467-471) edierten Ov̉@ßıxiov $\tau \alpha x \tau \iota x o ́ v$ (das sich, wie durch Förster bewiesen ward, als eine Kompilation aus Arrian entpuppt hat) - und zwar vor allem das folgende Epigramm:

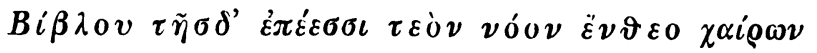

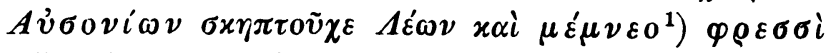

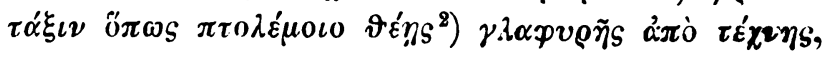

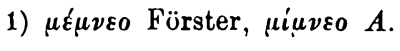
2) จรins $A$. 


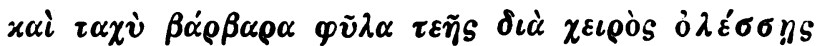
.

S. 463 a. a. 0 . bemerkt hierzu Förster, dab der in V. 2 angeredete

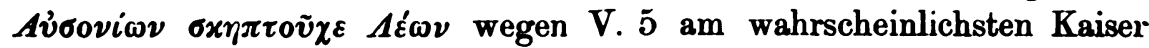
Leo VI der Taktiker sein dürfte. Das ist über allen Zweifel erhaben.

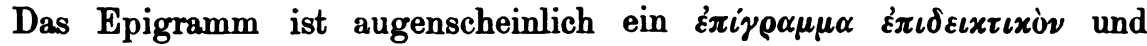
paBt ausgezeichnet auf das taktische Werk Leos. Ob es mit dem Kaiser gleichzeitig ist, wie Förster meint, bezweifle ich. Es hat den Anschein, daB es zu der Zeit entstand, in der das Epigramm auf fol. $352^{\mathrm{r}}$ entstanden ist, und das den $N \alpha v \mu \alpha \chi \iota x \dot{\alpha}$ des $B \alpha \sigma i \lambda \varepsilon \iota 0$ S $\pi \alpha \tau \rho i-$ $x \iota s$ vorangeht:

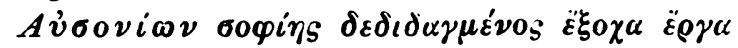

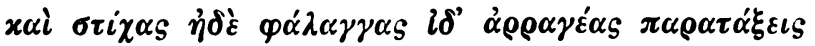

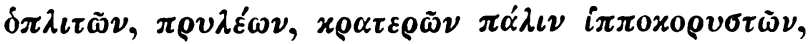

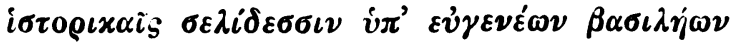

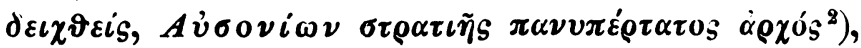

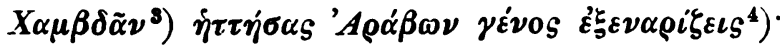

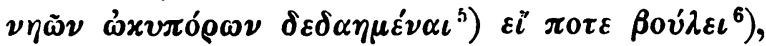

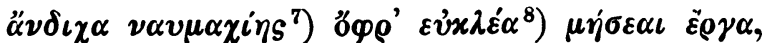

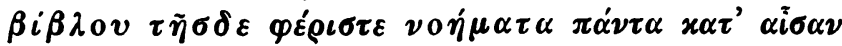

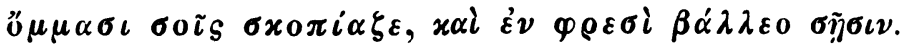

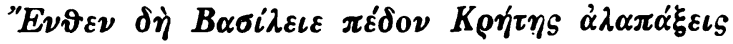

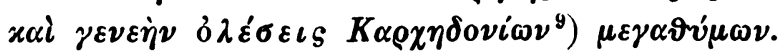

Das Epigramm führt in einer anderen $\mathrm{Hs}$, aus der es Brunck, Anal. III S. 277 nach Fabricius herausgegeben hat, den Vermerk $\varepsilon i_{S}$

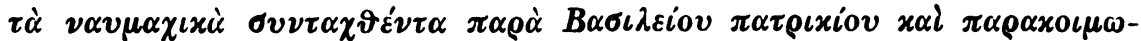
$\mu \varepsilon \dot{v o v}$, und Jacobs bemerkt hiezu im Zusammenhange mit Anth. Pal. IX 686: „Basilium quemdam invenio Protospatharium, qui circa ann. Chr. 956 multa praeclara gessit contra Arabas in Italia inferiore et Sicilia, ubi Arabum ducem, Ammar sive Ebn-Ammar magno praelio fudit." Um diese Zeit also werden wir jene Sammlung der griech. Kriegsschriftsteller ansetzen, die zum Grundstocke, wie er in Laur. LV 4 vorliegt, noch die auf das Seekriegswesen bezüglichen Schriften in sich geschlossen hat. Hat nun die Rezension $P_{1} R$ der Taktik Leos und die Sammlung, die auch die Schrift De velitatione bellica enthält,

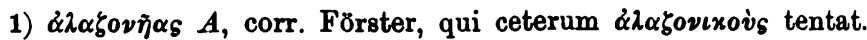

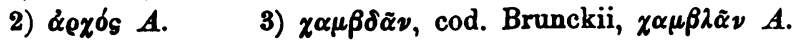

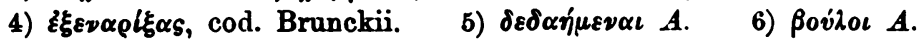

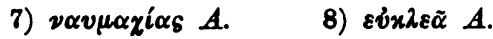

9) „Arabes intellige, qui Africae septentrionalem oram tunc imperio tenebant" (Cougny.). 
Nikephoros Uranos anfertigen lassen, so geht die Sammlung, die im Ambros. B 119 sup. vorliegt, auf die Verfügungen des siegreichen Feldherrn Basileios Patrikios zurück. Sie entstand also um die Mitte des 10. Jahrhunderts um die Zeit als der Zug gegen Kreta (s. Gelzer in Krumbachers GBL. ${ }^{2}$ S. 984) schon geplant war, - eventuell, falls wir es mit einem vaticinium post eventum zu tun haben, bald nachher. Unsere ganz besondere Aufmerksamkeit verdient im Epigramm die

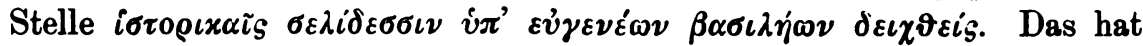
mindestens auf Kaiser Leo Bezug, in dessen Taktik (Prooem. § 5) wir

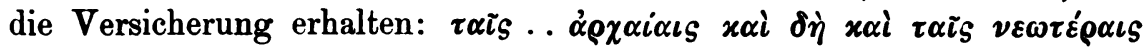

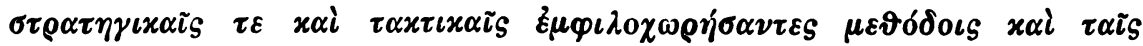

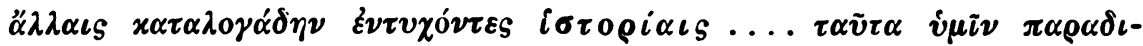

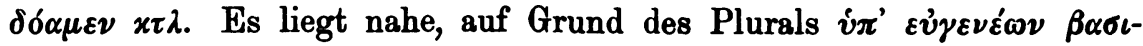
$\lambda \eta \dot{\eta} \omega \nu$ die Vermutung aufzustellen, daB zur Zeit des Basileios Patrikios mehr denn das eine Werk in der Sammlung unter eines Kaisers Namen gegangen ist. Das kann nur das Werk des sog. Maurikios gewesen

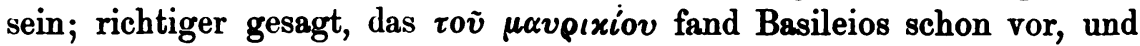
nur in einer späteren Abschrift der Sammlung, vielleicht erst in

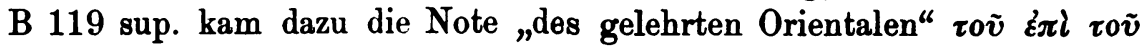

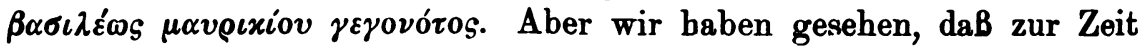
der Abschrift des Vindob. philol. gr. 275, also am Ende des 10. Jahrhunderts herum der Name nicht bekannt war. Wie er entstanden sein mag, werden wir weiter unten sehen. Vorerst kehren wir zu den zwei anderen Epigrammen zurück, von welchen noch nicht die Rede war. Das erstere von beiden lautet:

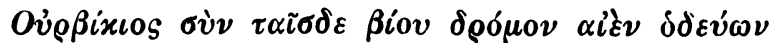

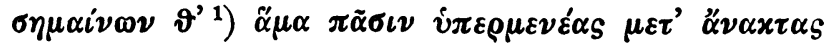

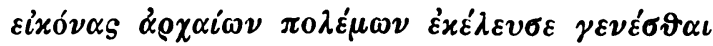

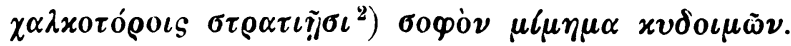

Dieses Epigramm kann nun keinesfalls auf das von Förster herausgegebene Machwerk, das $\tau \alpha x \tau \iota x o ́ v$, sich beziehen, noch weniger ist es

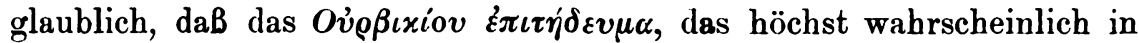
der Sammlung gar nicht gestanden hat, irgend wen zu einem Epigramme begeistert haben soll. Es kann sich nur auf das Werk des sog. Maurikios beziehen, das im Laurentianus LV 4 dem Urbikios zugeschrieben wird. Dann mag es um ein gutes Stück älter sein, als die andern zwei Epigramme aus der Zeit des Basileios Patrikios. Aber in die Sammlung muB das Epigramm von diesem aufgenommen worden
1) $\tau \varepsilon A$.

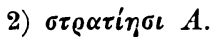


sein, denn es steht mit dem auf Leo bezüglichen Epigramm auf einer Seite, sowie auch unser viertes Epigramm:

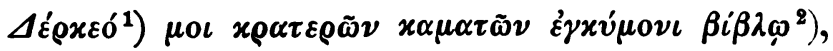

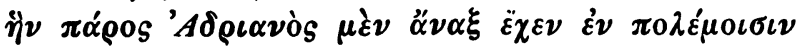

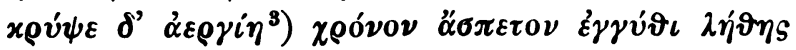

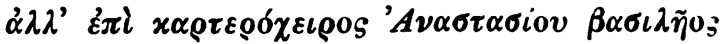

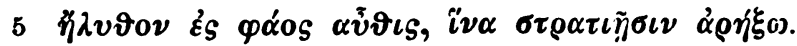

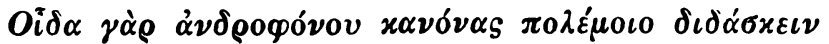

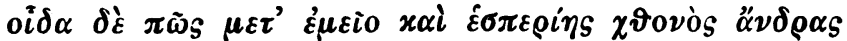

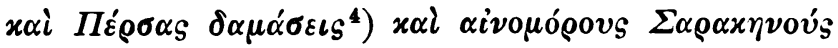

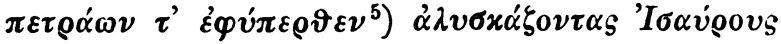

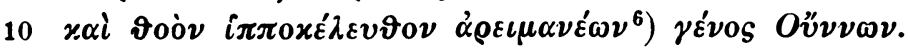

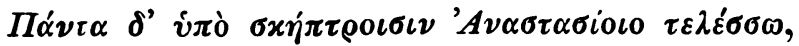

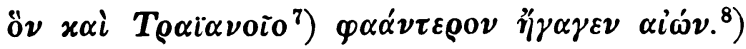

Auf was bezieht sich dieses Epigramm, und wann mag es entstanden sein?

Nach Förster (S. 452) ist das Zeugnis nicht hoch anzuschlagen;

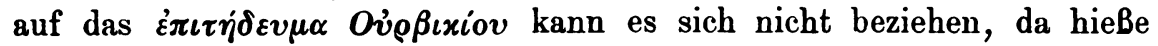

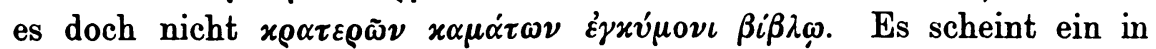
sich abgeschlossener Versuch zu sein, gemacht zur Ausfüllung einer in den Handbüchern der Taktik zur Zeit fühlbaren Lücke. Der Verf. mag Urbikios selbst sein, die Zeit die des Anastasios: die aufgezählten Völker sind es, deren sich das Reich unter seiner Regierung zu erwehren hatte.

Das ist alles sehr ansprechend. Aber was machen wir dann mit dem vorhergehenden Epigramm? Wir werden dann nicht klüger! Soviel steht fest, daB auch das zweite Epigramm sich nicht auf das höchst geringwertige Ov̉@ßıxiov $\tau \alpha x \tau \iota x o ́ v$ beziehen kann. Wenn wir aber annehmen, daß die Epigramme zu einer Zeit entstanden sind, als der sog. Maurikios noch unter dem Namen des Urbikios ging, und

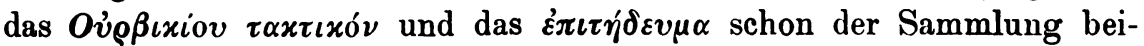
geschlossen waren, also um die Wende des 9. Jahrhunderts, stützen wir uns auf folgende Tatsachen: 1) die älteste Überlieferung des sog. Maurikios reicht im Laur. LV 4 in den Anfang des 10. Jahrhunderts

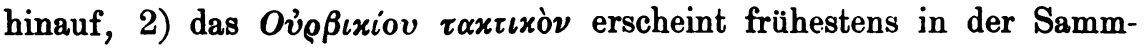
lung des Basileios Patrikios, das $\dot{\varepsilon} \iota \tau \eta \dot{\delta} \delta v \mu \alpha$ in der Sammlung des

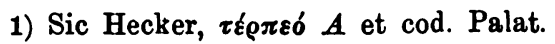

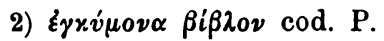

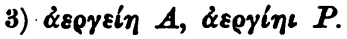

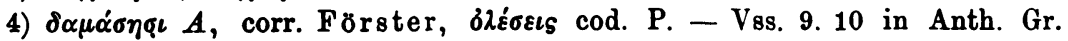
codice inverso ordine sunt traditi.

5) $\varepsilon \varphi^{\prime}$ ขึ $\pi \varepsilon \rho \vartheta \varepsilon \nu A$.

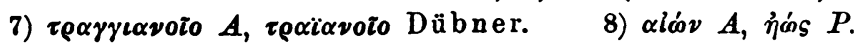


Nikephoros Uranos, deren Leo-Text jenen des Basileios zur Voraussetzung hat; beide Sammlungen enthalten das sog. Maurikiossche Werk. Es liegt daher nahe, bald nach der Sammlung $F V$ eine Abschrift anzunehmen, in der unser Maurikios noch unter dem Namen des Urbikios ging, das $\tau \alpha x \tau \iota x o ́ v$ und $\dot{\varepsilon} \pi \iota \tau \dot{\eta} \delta \varepsilon v \mu \alpha$ beigeschlossen und zur Ausfüllung einer entstandenen Lücke die beiden Epigramme gedichtet wurden. Diese beiden Epigramme verdrüngten dann von der Spitze des Werkes unseres Verf.s den Namen Urbikios; insbesondere das zweite Epigramm mag die Veranlassung gewesen sein, daB man den Namen des Urbikios strich und durch den des Maurikios ersetzte, den man als den mutmaßlichen Verfasser betrachtete, und der dem durch das Epigramm verdolmetschten Ansehen der Sammlung zu noch größerem Glanze verhalf. Da das Epigramm auch in der Anthologia Palatina steht, und zwar im IX. Buche (in den Ausgaben sub nro 210), stand es schon in der Anthologie des Konstantinos Kephalas (vgl. Sternbach, Melet. Graeca I [Wien 1886] S. 17 u. f.); es steht zwischen Epigrammen des Leo Philosophus (was freilich nicht viel bedeuten will), der Epigramme auf mathematische und geometrische Bücher gedichtet hat, wie

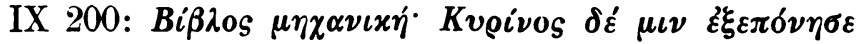

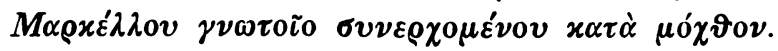

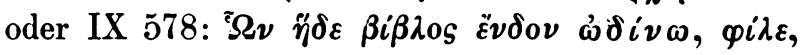

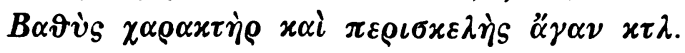

Er, der zur Zeit Leons des Weisen gelebt hat, kann ganz gut als Verfasser der zwei Urbikios-Epigramme angesehen werden.

Auf diese Art glaube ich die Tatsache, laut welcher das Maurikiossche Werk in der älteren Überlieferung unter dem Namen des Urbikios geht, mit der Angabe der jüngeren Überlieferung zusammengereimt zu haben.

Wenn nun die ältere Überlieferung das Werk unter dem Namen des Urbikios kennt, quid tuuc? Wir wissen ja nichts über diesen Urbikios! Wir wissen nur von einem Urbikios, der zur Zeit Anastasios' I gelebt hat.

Der Name kommt aber nicht singulär vor! Der oben S. 70 zitierte Anonymus sagt ja schon $\pi \alpha \varrho \dot{~ O v ̉ @ \beta \iota x i o v ~} \pi \alpha \tau \rho \iota x i o v ~ \ddot{\lambda} \lambda \lambda o v$

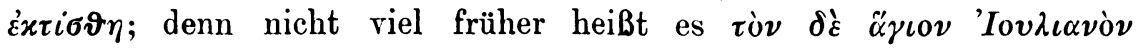

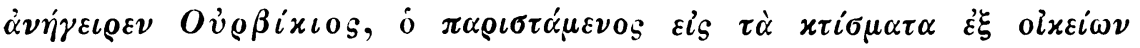

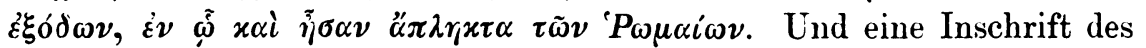
6./7. Jahrhunderts in Konstantinopel bezieht sich auf die Abgrenzung der Besitzungen eines Dexikrates und eines Urbikios (s. Byz. Z. IX [1900] S. 717). Der Name ist also unter allen Umständen nicht zurückzuweisen. Er konnte einem Militär angehören, der zuerst 
alles gesammelt hat und in einem Corpus vereinigt, was sich auf die Kriegswissenschaft bezogen hat, ja selbst sogar manches dazugeschrieben. In dieser Eigenschaft, als erster Sammler kriegswissenschaftlicher Schriften mag er der Zeit Leos vorgeschwebt haben; daB ihm da manches fragmentarisch aussehende Traktätchen

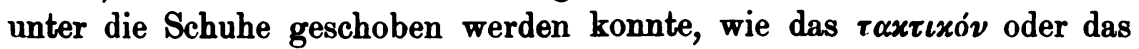

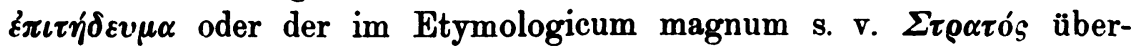
lieferte Artikel, ist nichts Unmögliches.

Da taucht aber die Frage auf, warum Leo ihn nicht bei seinem Namen nennt, während doch Arrian, Aelian und Onasander auch dem Namen nach angeführt sind?

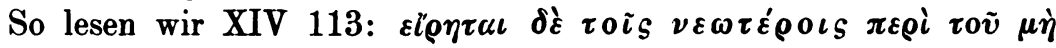

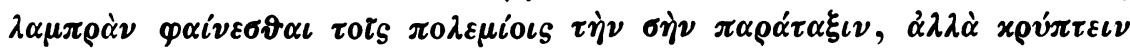

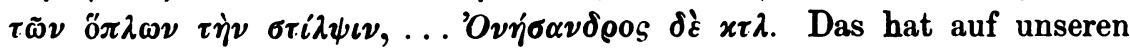
sog. Maurikios, oder jetzt schon Urbikios, Bezug, wo man diese Forderung VII 17, 12 (p. 165 u. f. Sch.) erhoben findet (vgl. auch Leo

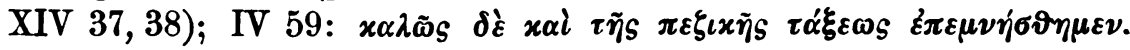

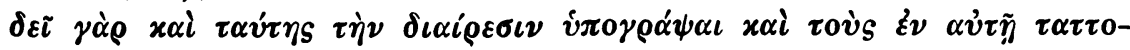

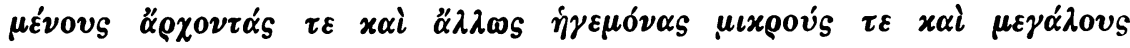

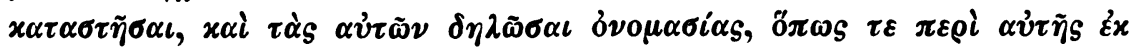

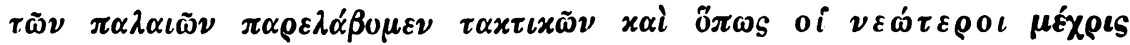

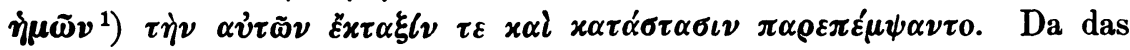
hierauf Folgende zum großen Teile aus Urbic. XII 8,8 und XII 8,9

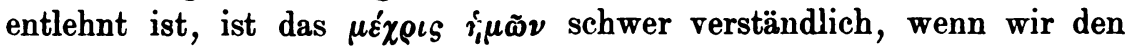
Försterschen Standpunkt einnehmen. Hinwieder aber im Sinne der jüngeren Rezensionen die Stelle aufzufassen verbietet uns XV 28, -

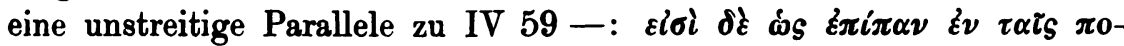

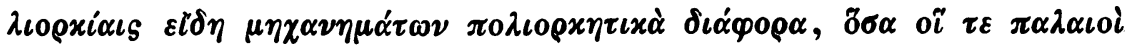

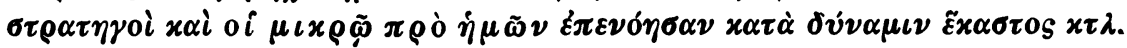
Nachdem von den hierauffolgenden $\$ \S 47-53,55-62,64,67-77$ aus Urbikios entnommen sind, haben wir es hier mit einem eminent wichtigen Zeugnisse zu tun. Auch XV 1 schickt Leo die

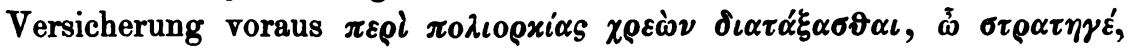

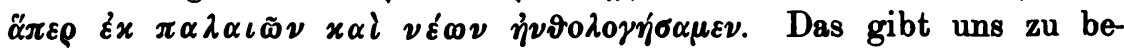
denken, wenn wir die sog. Maurikiossche Taktik so weit als möglich zurückdatieren wollen.

Wie wir sehen, spricht Leo immer in der Mehrzahl von jüngeren Taktikern da, wo wir nur Urbikios als Quelle nachweisen können.

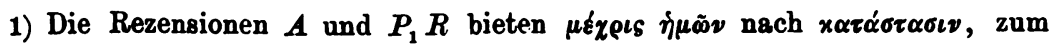
Zeichen, $\mathrm{da}_{\mathrm{B}}$ sie den Satz anders verstanden wissen wollen. Die Rez. pv bietet:

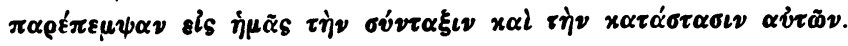


Da liegt die Möglichkeit nahe anzunehmen, daB Leo in dem Werke des Urbikios eine Sammelarbeit gesehen hat. Sagt uns doch am Ende

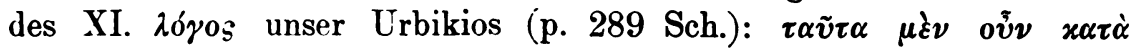

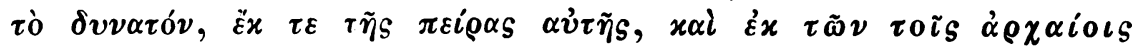

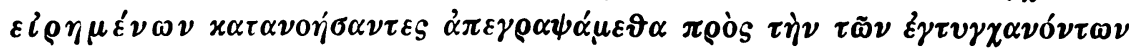

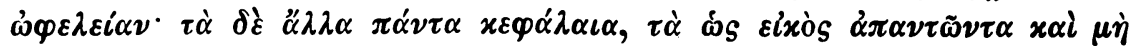

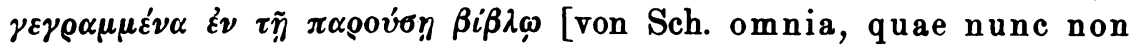
occurrunt, nec in libro hoc descripta sunt übersetzt] $\tilde{\varepsilon} \chi \tau \varepsilon \tau \tilde{\varepsilon} \nu$

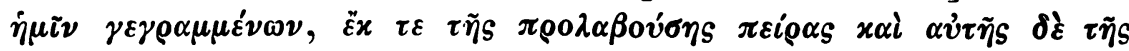

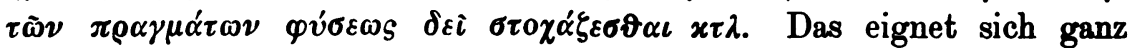
schön für einen SchluBparagraphen zu einem größeren Werke. Freilich

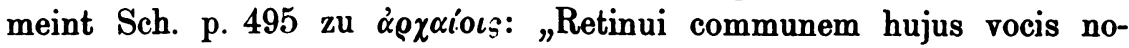
tionem. Dubito nihilominus, annon praefectos militum intelligat, e quibus talia cognoverit. Scribit sane hic de ratione subigendi Antas, quos sub Iustiniano demum invasisse imperium Romanum, noscique coepisse alibi ostendi. At á $\varrho \alpha i_{i o v}$ posse dici, qui de ipsis aliquid prodiderunt Iustiniani vel sequentibus temporibus, ego quidem ambigo." Aber wäre dies nicht wieder ein Beweis, dab das sog. Maurikiossche Werk weiter herabzurücken wäre? Dann werden wir natürlich nicht mehr für die Selbständigkeit des Werkes einstehen; Krumbachers GBL $^{2}$ S. 636 ausgesprochene Ansicht: „Das Buch ist eine ziemlich unselbständige Kompilation" gewinnt an Bedeutung. Es scheint ein Werk zu sein, wie das des Leo, geht aber bis zu Aelian und Arrian nicht hinauf.

Wie das Leosche Werk, mag auch dieses einen Grundstock gehabt haben. Diesen Grundstock entdeckt zu haben, ist das Verdienst Zachariaes v. Lingenthal, der aber freilich das ganze, unter dem Namen des Maurikios, resp. Urbikios gehende Werk einem Rufus zuschreibt (a. a. O. S. 455 u. f.).

Nach Z. v. L. erkunnte man im 9. Jahrhundert, daB die Vorschriften der Digesten und des Codex Iustiniani nicht eigentlich aufgehoben seien. Dies mag Juristen veranlaßt haben, Stellen aus den Digesten und dem Codex mit den betreffenden Abschnitten aus den Strategica und den Tactica zu einem Strafrecht zu kombinieren, wie wir es als Leges militares in zahlreichen Hss der kleinen kaiserlichen Manualia iuris und ihrer Bearbeitungen im Anhang finden. Die zahlreichen $\mathrm{Hss}$, welche die Leges militares oder Auszüge daraus geben, bedienen sich zur Bezeichnung der Stücke aus den Strategica und den Tactica ausnahmslos der Überschrift $\dot{\varepsilon} x \tau \tilde{\omega} \nu$ @ov́ $x \tilde{\omega} \nu$. Mithin wird in den Leges militares Rufus (der Name deutet auf okzidentalen Ursprung) als Verfasser der Strategica bezeichnet. 
Dies die Ansicht Z.s v. L., und da er erweisen kann, daB 15 Kapitel der Leges militares und zwar im Anfang sich in Inhalt und Form mit 15 Paragraphen der Tactica-Strategica decken, ist der SchluB gestattet, daB im Werke des Urbikios eine gewisse Partie von Rufus stammt, oder Urbikios identisch mit Rufus ist.

Um dies entscheiden zu können, sei mir gestattet einige Kapitel aus den Leges militares sowohl, wie aus den beiden Fassungen unseres Urbikios, endlich aus den Tactica Leonis in Gegenüberstellung abzudrucken:

\section{Leg. Mil.}

„Urbikios"

(fol. $10^{\mathrm{v}}$ )

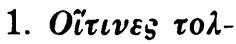

„Maurikios"

(fol. $126^{\mathrm{r}}$ )

$E i \quad \tau \iota \nu \varepsilon_{S} \quad \tau 0 \lambda$ -
Leo VIII 5.

$E l \delta \varepsilon^{\prime} \tau \iota \nu \varepsilon \subseteq \tau 0 \lambda-$

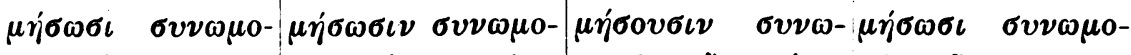

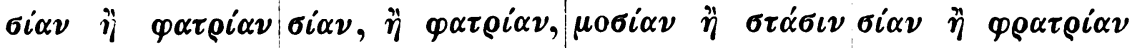

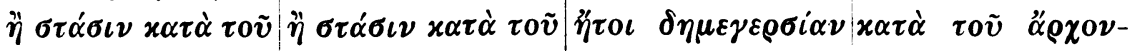

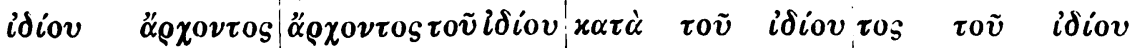

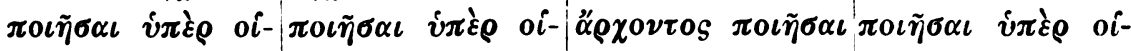

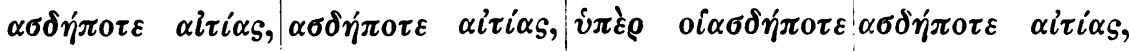

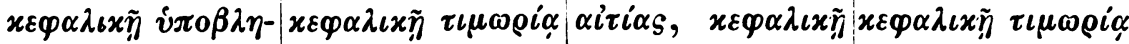

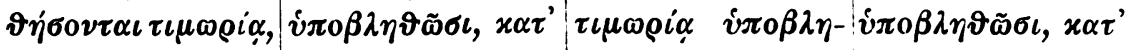

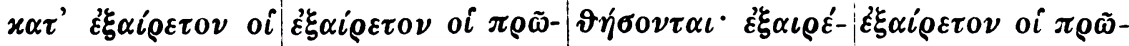

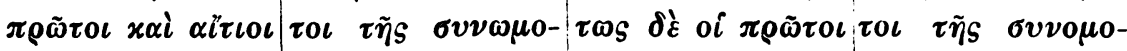

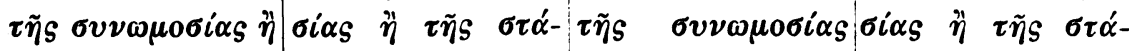

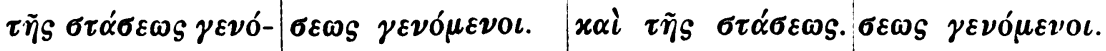
$\mu \varepsilon \nu 0 \iota$.

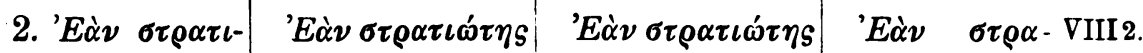

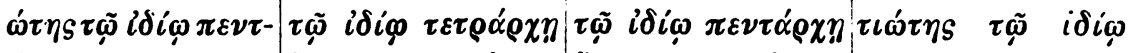

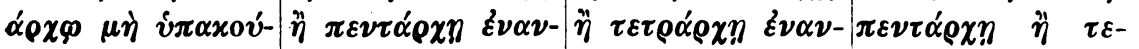

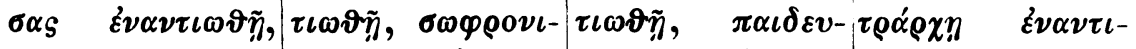

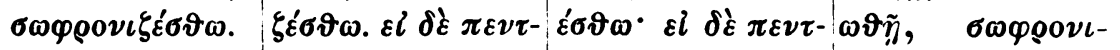

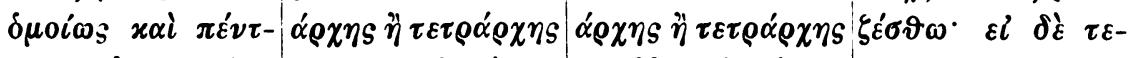

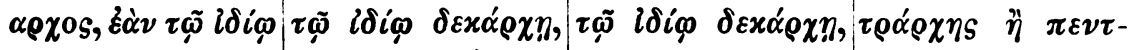

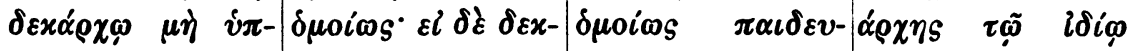

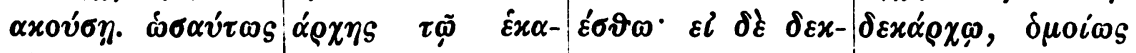

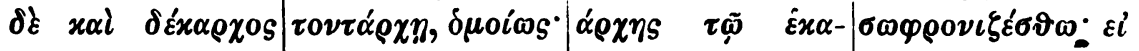

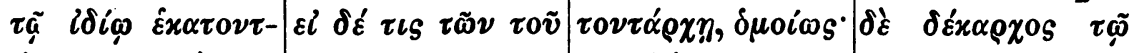

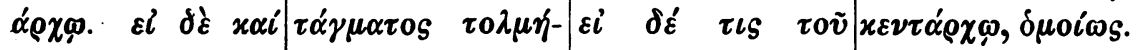

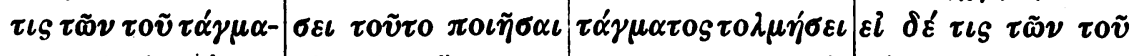

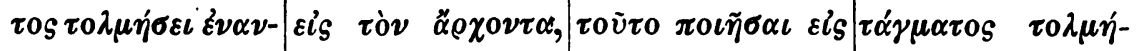

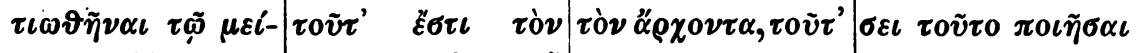

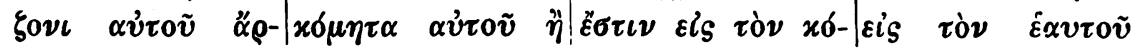




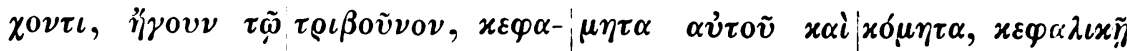

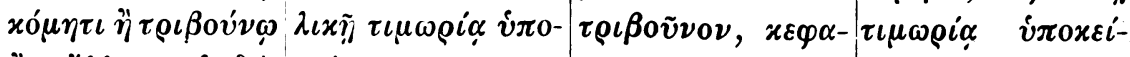

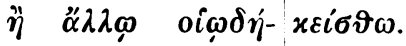
$\pi 0 \tau \varepsilon, \tau \tilde{\eta} \dot{\varepsilon} \sigma \chi \alpha \dot{\tau} \eta$

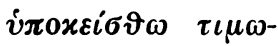

pía. $\lambda \iota x \tilde{\eta} \tau \iota \mu \omega \rho i \alpha \dot{v} \pi 0-\sigma \vartheta \omega$. $\varepsilon \dot{i \sigma \vartheta \omega . ~}$

fol. $11^{\mathrm{r}}$.

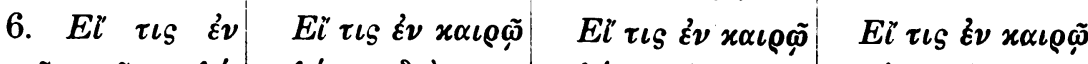

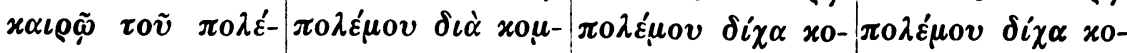

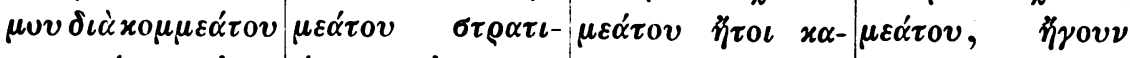

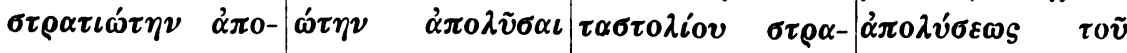

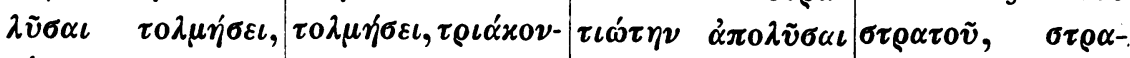

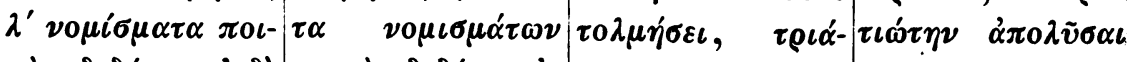

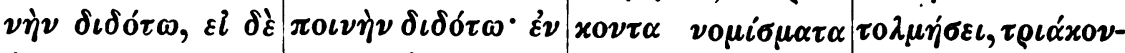

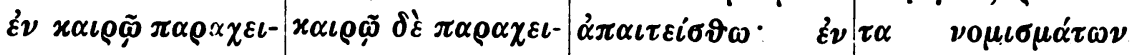

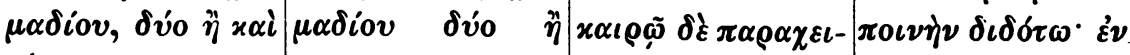

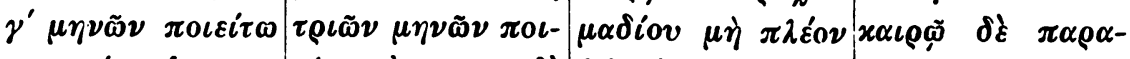

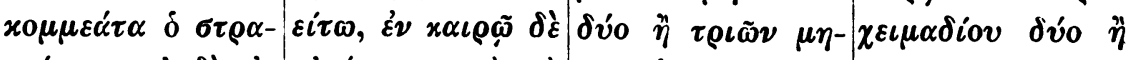

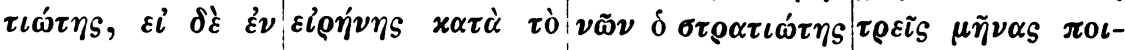

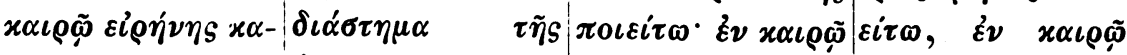

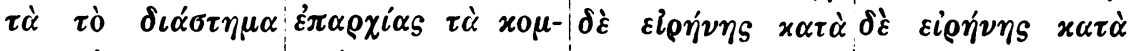

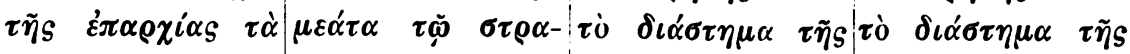

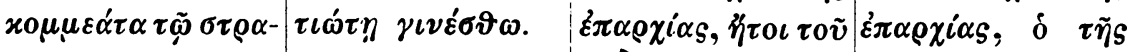

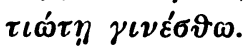

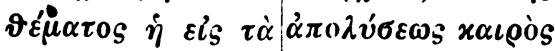

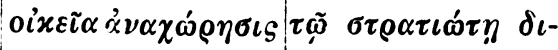

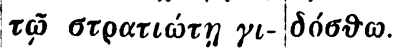

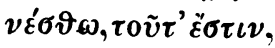

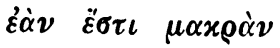
$\tau \dot{v} \theta \dot{u} \mu \alpha \mu \dot{v} \tau \Delta \tilde{v}, \ddot{i} \nu \mu$

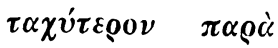
$\tau o \grave{v} s \dot{\varepsilon} \gamma \gamma \dot{v} \boldsymbol{S} 0 i x o \tilde{v} \nu$ $\tau \alpha_{S} \dot{\alpha} \pi 0 \sigma \tau \varepsilon^{\prime} \lambda \lambda r \tau \alpha \iota$.

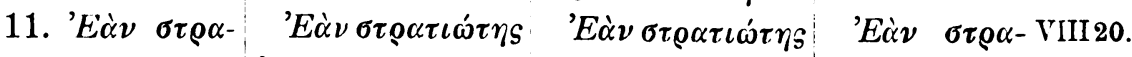

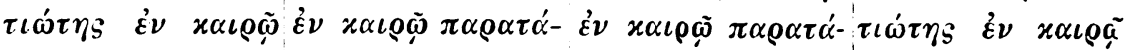

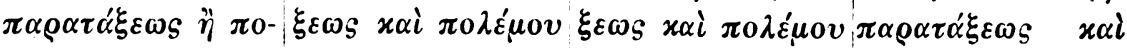

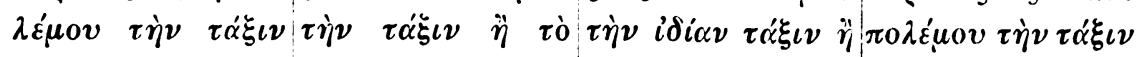

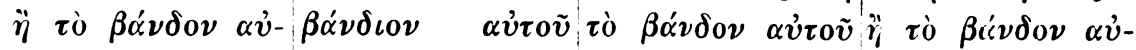

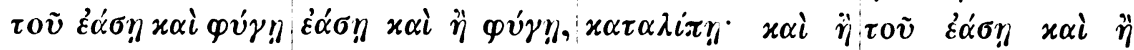

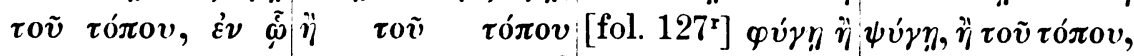

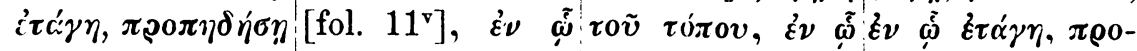

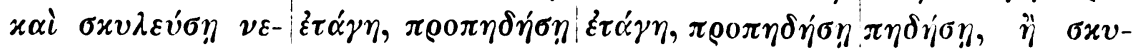




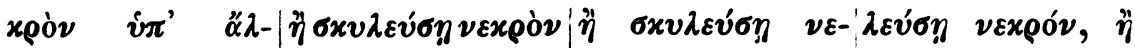

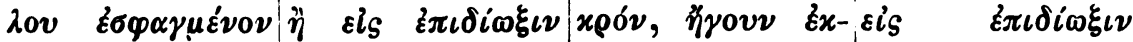

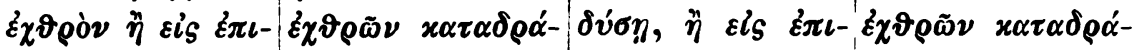

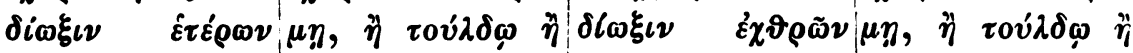

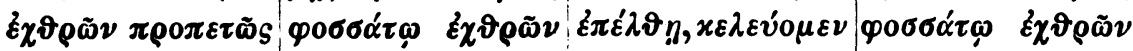

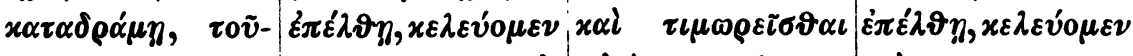

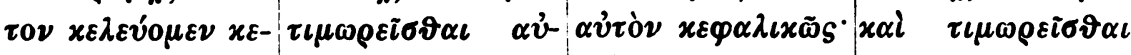

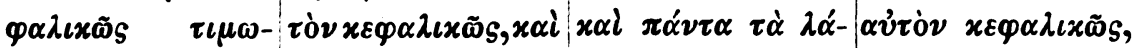

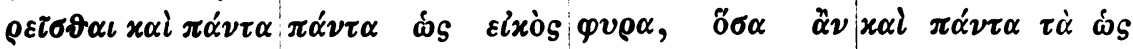

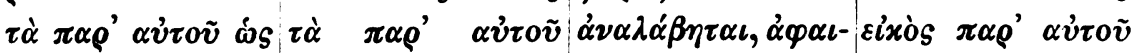

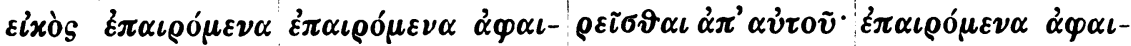

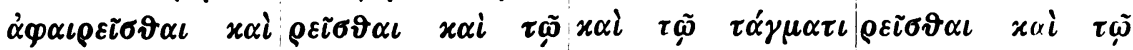

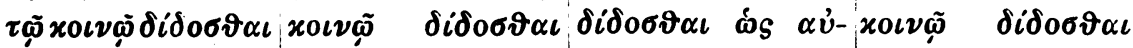

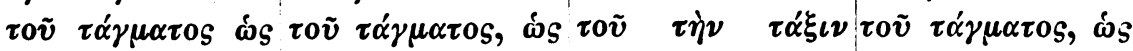

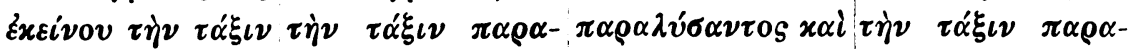

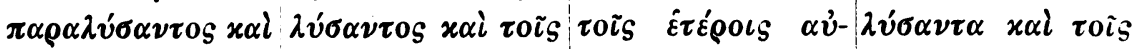

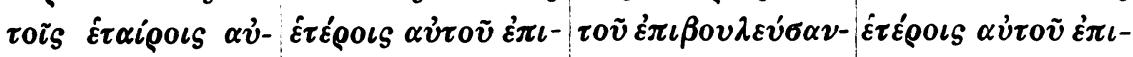

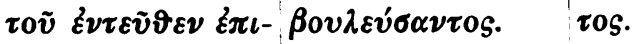
$\beta 0 v \lambda \varepsilon v \sigma \sigma \nu \tau \alpha$.

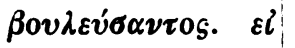

$\delta \grave{\varepsilon} x \alpha \grave{\imath} \varphi \iota \lambda \alpha \nu \vartheta \rho \omega-$

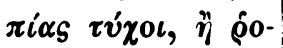

$\pi \alpha \dot{\lambda} 0 \iota s \quad \tau v \pi \tau \varepsilon \dot{\sigma} \sigma \omega$

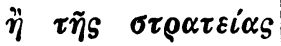

$\varepsilon v \alpha \lambda \lambda \alpha \tau \tau \varepsilon \dot{\tau} \tau \omega$.

Lesen wir diese Gegenüberstellungen aufmerksam durch, so ist das erste, was sich konstatieren läBt, daß die Taktik Leos mit den Leges militares nicht zusammengehört. Die Taktik Leos fuBt ganz auf der florentinischen Fassung des sog. Maurikios. Die zweite Frage wäre die, ob die ambrosianische Fassung den Loges wilitares nicht nahe. steht? Auch hierauf werden wir eine negative Antwort geben müssen. Es besteht also demnach nur der Konnex mit der florentinischen Fassung. Da die Leges militares schon im Codex Bodleianus i15, Laud. 73, vom Jahre 903 enthalten sind, (s. Z. v. L. a. a. O. S. 438) müssen sie schon im 9. Jahrhundert zusammengestellt worden sein. Für diese spätere Zeit zeugt auch die Zutat (in § 11) $\varepsilon i \quad \delta \dot{c}$ xai $\varphi \iota-$

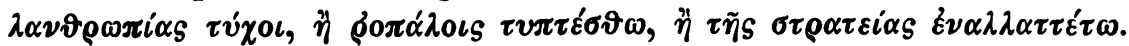
Aber in sprachlicher Beziehung scheinen die Leges militares ebenso, wie die florentinische Fassung auf dasselbe Original zurückzugehen.

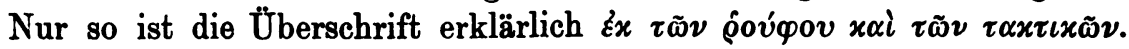
Zachariae r. Lingenthal meinte a. a. O., daB unter den $\tau \alpha x \tau \iota x \alpha ́$ die des Leo zu verstehen sind und diese leoninische Taktik von Leo dem 
Isaurier herrührt, aber ich habe - und gleichzeitig mit mir Kulakovskij (s. B. Z. VIII [1898] S. 256) - bewiesen ${ }^{1}$ ), daB die unter dem Namen Kaiser Leos überlieferte Taktik nur von Leo VI herstammen kann. Also beziehen sich die $\tau \alpha \varkappa \tau \iota x \alpha^{\prime}$ auf die Urbikiossche Taktik, die schon dazumal als ein Sammelwerk angesehen worden sein mag. Was nun alles im Werke des Urbikios aus Rufus stammen mag, bleibt für spätere Forschungen vorbehalten. Nur eine neue, auf Kenntnis sämtlicher Hss fußende Ausgabe, die wiederum die Ausgabe der Tactica Leonis zu ihrer Vorbedingung hat, kann uns hier heller sehen machen. DaB in der Taktik des Urbikios fremde Bestandteile sind, haben wir gesehen. $\mathrm{DaB}$ das Werk noch ärger zusammengeflickt ist, als die Kompilation Leos, zeigt der $\pi i \nu \alpha \xi$, der ihm vorangeht. Ja selbst der Grundstock, der Rufus, scheint eine Kompilation gewesen zu sein. Darauf weist schon hin, was wir bei Sch. p. 449 zu der Stelle $\dot{\varepsilon} \nu \tau \tilde{a}$

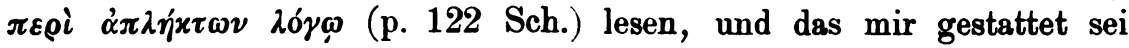
hier per longum et latum anzuführen: "Quis hic $\lambda o^{\prime} \sigma_{0} \pi \varepsilon \rho l ~ \alpha \dot{\pi} \lambda \dot{\eta} x \tau \omega \nu$ ? In Mauricio toto nullum istiusmodi invenio vel librum vel caput, praeter unicum caput IX libri VII [ein Kapitel von wenigen Zeilen],

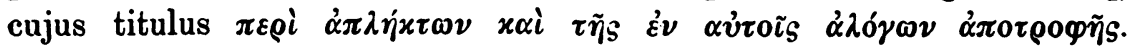
Sed in eo capite nil legitur, quod huc quadret; de cura colligendi pabuli solum agitur. Quid dicemus? An scripsisse nostrum alio et peculiari opere de castris? Non existimo. Quin potius caput sequens tertium innuere opinor. Ibi enim omnia occurrunt, quae huc spectant. At alius est capitis illius titulus. Nempe Leonem est secutus, qui

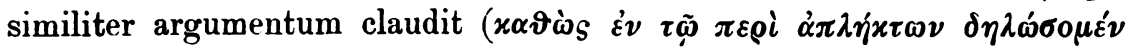
боє $\tau \dot{ } \boldsymbol{\pi} \omega\left[\begin{array}{ll}\mathrm{X} & 6\end{array}\right]$ ). Docet opinor satis evidenter vel hic locus, habuisse nostrum Tactica Leonis in manibus, cum ista scriberet. Quamquam non probare possim, quod rejiciat lectorem ad $\pi \varepsilon \rho i \dot{\alpha} \pi \lambda \dot{x} x \tau \omega \nu$

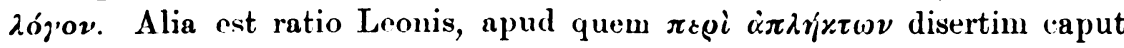
undecimum inscribitur."

Man sieht, wie mannigfaltige Fragen hier aufgerollt werden können. Ich bin zufrieden, wenn ich das Interesse Berufener für das Studium

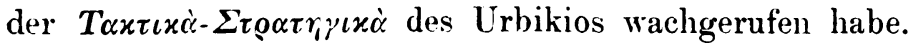
Budapest.
R. Vári.

1) M. Mitard, Études sur lè règne de Léon VI (Byz. Z. XII S. 585 ff.) rennt schon offene Türen ein. 